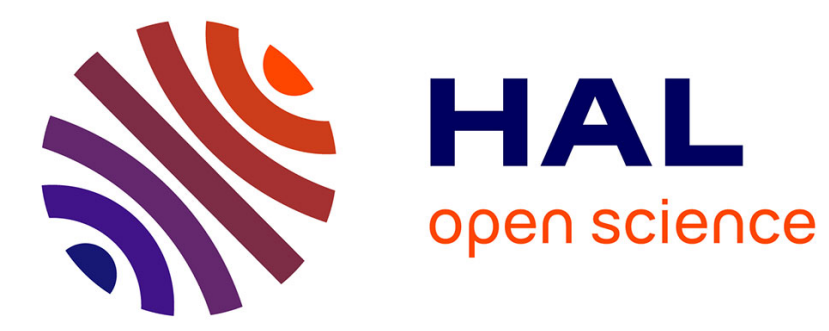

\title{
Cylinders in Fano varieties
}

\author{
M Zaidenberg, Ivan Cheltsov, Jihun Park, Yuri Prokhorov
}

\section{To cite this version:}

M Zaidenberg, Ivan Cheltsov, Jihun Park, Yuri Prokhorov. Cylinders in Fano varieties. EMS Surveys in Mathematical Sciences, 2021, 8, pp.39 - 105. 10.4171/emss/44 . hal-03429687

\section{HAL Id: hal-03429687 https://hal.science/hal-03429687}

Submitted on 15 Nov 2021

HAL is a multi-disciplinary open access archive for the deposit and dissemination of scientific research documents, whether they are published or not. The documents may come from teaching and research institutions in France or abroad, or from public or private research centers.
L'archive ouverte pluridisciplinaire HAL, est destinée au dépôt et à la diffusion de documents scientifiques de niveau recherche, publiés ou non, émanant des établissements d'enseignement et de recherche français ou étrangers, des laboratoires publics ou privés. 


\section{Cylinders in Fano varieties}

\section{Ivan Cheltsov, Jihun Park, Yuri Prokhorov, and Mikhail Zaidenberg}

Abstract. This paper is a survey about cylinders in Fano varieties and related problems.

Throughout this paper except for Section 4.3, we always assume that all varieties are defined over an algebraically closed field $\mathbb{k}$ of characteristic 0 .

\section{Introduction}

A cylinder in a projective variety $X$ is a Zariski open subset $U \subset X$ such that

$$
U \cong \mathbb{A}^{1} \times Z
$$

for an affine variety $Z$. If $X$ contains a cylinder, we say that $X$ is cylindrical. Since cylindrical varieties have negative Kodaira dimension, we will focus our attention on cylindrical Fano varieties, because they are building blocks of projective varieties with negative Kodaira dimension.

Example 1.1. For positive integers $m, n$ with $m<n$, let $X$ be the $\operatorname{Grassmannian} \operatorname{Gr}(m, n)$ of $m$-dimensional subspaces of an $n$-dimensional vector space over $\mathbb{k}$. Then $X$ is a smooth projective variety of dimension $m(n-m)$, and $-K_{X} \sim n H$, where $H$ is an ample generator of the group $\operatorname{Pic}(X)$. Since $X$ contains an open Schubert cell isomorphic to $\mathbb{A}^{m(n-m)}$, it is a cylindrical Fano variety.

However, not all Fano varieties are cylindrical, e.g. smooth cubic threefolds and smooth quartic threefolds do not contain cylinders, because they are irrational $[48,109]$. On the other hand, every smooth rational projective surface contains a cylinder (see, for example, [120, Proposition 3.13]). In particular, all smooth del Pezzo surfaces (two-dimensional Fano varieties) are also cylindrical. Therefore, one can expect that all rational Fano varieties are cylindrical. However, the following example shows that this is not the case:

Example 1.2. Let $X$ be a hypersurface of degree 6 in $\mathbb{P}(1,1,2,3)$ that is given by

$$
x_{3}^{2}=x_{2}\left(x_{2}+x_{0} x_{1}\right)\left(x_{2}+\lambda x_{0} x_{1}\right),
$$

2020 Mathematics Subject Classification. Primary 14E05, 14J45, 14J50, 14R20, 14R25; Secondary 14E08, $14 \mathrm{E} 30$.

Keywords. Cylinder, Fano variety, unipotent group action. 
for some $\lambda \in \mathbb{k} \backslash\{0,1\}$, where $x_{0}, x_{1}, x_{2}$ and $x_{3}$ are coordinates of weights $1,1,2$, and 3, respectively. Then $X$ is a del Pezzo surface that has exactly two Du Val singular points of type $\mathrm{D}_{4}$, it is rational, has Picard number 1 , and does not contain cylinders by [39, Theorem 1.5], see also Theorem 2.10 of the present survey and its proof.

The surface in Example 1.2 is singular. There are other examples of singular noncylindrical rational surfaces (see Examples 1.27, 2.5, and 2.6 below). What about smooth rational varieties?

Question 1.3. Does every smooth rational Fano variety contain a cylinder?

We do not know the answer to this question even in dimension three despite the fact that smooth three-dimensional Fano varieties (Fano threefolds) are completely classified and well studied [113]. Nevertheless, we believe that the answer to Question 1.3 is negative (see Conjectures 3.9 and 3.13). In fact, we do not know the answer to the following generalization of Question 1.3:

Question 1.4 ([33]). Is it true that any smooth rational variety is cylindrical?

A cylindrical variety $X$ is birationally equivalent to a product $\mathbb{A}^{1} \times Z$. Thus, if $X$ is rationally connected, then $Z$ is also rationally connected. In particular, if $X$ is a cylindrical Fano threefold with Kawamata log terminal singularities, then $X$ must be rational [215]. Moreover, we have the following proposition.

Proposition 1.5. Let $X$ be a cylindrical smooth Fano variety with $\rho(X)=1$. Then $X$ is birational to the product $Y \times \mathbb{A}^{2}$ for some rationally connected variety $Y$.

Proof. Let $U$ be a cylinder in the Fano variety $X$. Then $U \cong Z \times \mathbb{A}^{1}$ for some affine variety $Z$. Let $\bar{Z}$ be a projective completion of the variety $Z$. Consider the natural completion

$$
\bar{Z} \times \mathbb{A}^{1} \subset \bar{Z} \times \mathbb{P}^{1},
$$

let $D=\left(\bar{Z} \times \mathbb{P}^{1}\right) \backslash\left(\bar{Z} \times \mathbb{A}^{1}\right)$, and let $\psi: \bar{Z} \times \mathbb{P}^{1} \rightarrow X$ be the birational map induced by the open embedding $Z \times \mathbb{A}^{1} \subset X$. Since $\rho(X)=1$ by assumption, the divisor $D$ must be $\psi$-exceptional, which implies that $D$ is birational to $Y \times \mathbb{A}^{1}$ for some variety $Y$. Then $X$ is birational to $Y \times \mathbb{A}^{2}$. Since $X$ is rationally connected (see [26,130]), the variety $Y$ is rationally connected as well.

Corollary 1.6. Let $X$ be a cylindrical smooth Fano fourfold with $\rho(X)=1$. Then $X$ is rational.

However, we do not know cylindricity of many rational smooth Fano fourfolds of Picard rank 1. For instance, we do not know whether any smooth rational cubic fourfold in $\mathbb{P}^{5}$ is cylindrical or not (see Question 3.18 and Remark 3.19). Keeping in mind Corollary 1.6, we ask:

Question 1.7. Is it true that all cylindrical smooth Fano varieties of Picard rank one are rational? 
In the paper [89], Gromov asked whether every smooth rational variety is uniformly rational? Recall from $[21,145,174]$ that a smooth rational variety is said to be uniformly rational if its every point has a Zariski open neighborhood isomorphic to an open subset of the space $\mathbb{A}^{n}$ (cf. [20]). Similarly, a smooth cylindrical projective variety is said to be uniformly cylindrical if its every point is contained in a (Zariski open) cylinder (see Section 4.1 for the motivation and examples). It is easy to see that all smooth rational surfaces are uniformly rational and uniformly cylindrical. On the other hand, we do not know the answer to Gromov's question for varieties of higher dimensions, and we do not know the answer to:

Question 1.8. Is it true that any cylindrical smooth projective variety is uniformly cylindrical?

In Section 3, we will present several cylindrical smooth Fano threefolds whose Picard groups are generated by their anticanonical divisors. We do not know such examples in any other dimension. The counter-examples to [194, Conjecture 5.1] found in [27] made us believe that such examples should exist in any dimension $\geqslant 4$. Therefore, we pose:

Problem 1.9. Find a cylindrical smooth Fano variety of dimension $\geqslant 4$ whose Picard group is generated by its anticanonical divisor.

One can also define cylindricity and uniform cylindricity for affine varieties in the same way we did this for projective varieties. Note that [120, Definition 3.4] asks that the cylinder should be principal, that is, its complement should be a principal divisor, which is not automatic.

Remark 1.10 (cf. Question 1.8). There are cylindrical smooth affine varieties that are not uniformly cylindrical. Indeed, let $V$ be the Koras-Russell cubic threefold in $\mathbb{A}^{4}$ that is given by

$$
x_{1}+x_{1}^{2} x_{2}+x_{3}^{2}+x_{4}^{3}=0,
$$

where $x_{1}, x_{2}, x_{3}$ and $x_{4}$ are coordinates on $\mathbb{A}^{4}$. Then $V$ is a cylindrical smooth affine variety [132]. Moreover, it follows from [62, Corollary 4.5] that $(0,0,0,0)$ is fixed by any element of $\operatorname{Aut}(V)$, which implies that this point is not contained in any cylinder in $V$. Indeed, otherwise the origin would be moved by a suitable $\mathbb{G}_{\mathrm{a}}$-action on $V$, cf. Theorem 1.13 below.

Like in the projective case, every cylindrical affine variety $X$ has negative log Kodaira dimension. Moreover, a smooth affine surface contains a cylinder if and only if its $\log$ Kodaira dimension is negative [150, Ch. 2, Theorem 2.1.1], cf. [151]. However, this is no longer true in higher dimensions:

Example 1.11. Let $X$ be a smooth hypersurface in $\mathbb{P}^{n}$ of degree $n \geqslant 3$. Then $\mathbb{P}^{n} \backslash X$ is a smooth affine $n$-fold of negative Kodaira dimension that does not contain cylinders [33, 56]. 
The problem of existence of cylinders in projective varieties is closely related to unipotent actions on the affine cones over them. To illustrate this link, consider the following question:

Question 1.12 ([64, Question 2.22]). Let $V$ be the affine cone in $\mathbb{A}^{4}$ over the Fermat cubic surface, which is given by

$$
x_{1}^{3}+x_{2}^{3}+x_{3}^{3}+x_{4}^{3}=0,
$$

where $x_{1}, x_{2}, x_{3}$ and $x_{4}$ are coordinates on $\mathbb{A}^{4}$. Does $V$ admit an effective $\mathbb{G}_{\mathrm{a}}$-action?

The answer to this question is negative [38], see also [51, Theorem 7.1] for a purely algebraic proof. The geometric proof of this fact is based on the following result:

Theorem 1.13 ([120, Proposition 3.1.5]). An affine variety $V$ admits an effective $\mathbb{G}_{\mathrm{a}}$-action if and only if $V$ contains a principal effective divisor $D$ such that $V \backslash \operatorname{Supp}(D)$ is a cylinder.

Using this criterion, we can formulate the corresponding criterion for projective varieties, which requires the following refined notion of cylindricity:

Definition 1.14. Let $X$ be a projective normal variety that contains a Zariski open cylinder $U$, and let $H$ be an ample $\mathbb{Q}$-Cartier $\mathbb{Q}$-divisor on $X$. The cylinder $U$ is said to be $H$-polar if

$$
U=X \backslash \operatorname{Supp}(D)
$$

for some effective $\mathbb{Q}$-divisor $D$ on the variety $X$ such that $D \sim_{\mathbb{Q}} H$.

Now, we are in a position to state the following criterion discovered in [121], see also [46].

Theorem 1.15. Let $X$ be a projective normal variety, let $H$ be an ample Cartier divisor on it, let

$$
V=\operatorname{Spec}\left(\bigoplus_{n \geqslant 0} H^{0}\left(\mathcal{O}_{X}(n H)\right)\right) .
$$

Then $V$ admits an effective $\mathbb{G}_{\mathrm{a}}$-action $\Longleftrightarrow X$ contains an $H$-polar cylinder.

Corollary 1.16. Let $X$ be a smooth rational projective surface. Then there is an embedding $X \hookrightarrow \mathbb{P}^{n}$ such that the affine cone in $\mathbb{A}^{n+1}$ over $X$ admits an effective $\mathbb{G}_{\mathrm{a}}$-action.

Corollary 1.17. Let $X$ be a projective normal variety in $\mathbb{P}^{n}$ whose divisor class group is of rank 1 . Then the affine cone in $\mathbb{A}^{n+1}$ over $X$ admits an effective $\mathbb{G}_{\mathrm{a}}$-action $\Longleftrightarrow X$ is cylindrical.

Remark 1.18. Let $X, H$ and $V$ be as in Theorem 1.15. If $V$ is $\mathbb{Q}$-Gorenstein and admits an effective action of the additive group $\mathbb{G}_{\mathrm{a}}$, then $X$ is a Fano variety and $H \sim_{\mathbb{Q}}-\lambda K_{X}$ for some $\lambda \in \mathbb{Q}_{>0}[120$, (3.18)]. This explains our primary interest in the affine cones over Fano varieties.

The problem of existence of an effective $\mathbb{G}_{\mathrm{a}}$-action on affine varieties is interesting on its own. If an affine variety $V$ admits a non-trivial $\mathbb{G}_{\mathrm{a}}$-action and $\operatorname{dim}(V) \geqslant 2$, then $\operatorname{Aut}(V)$ 
is infinite dimensional and non-algebraic [65]. On the other hand, if it does not admit nontrivial $\mathbb{G}_{\mathrm{a}}$-actions, then $\operatorname{Aut}(V)$ contains a unique maximal torus $\mathbb{T}$, and $\operatorname{Aut}(V)$ is an extension of its centralizer by a discrete subgroup in $\mathrm{GL}_{r}(\mathbb{Z})$ (see [10] for details).

Example 1.19. Let $V$ be the Pham-Brieskorn surface in $\mathbb{A}^{3}$, which is given by

$$
x_{1}^{a_{1}}+x_{2}^{a_{2}}+x_{3}^{a_{3}}=0,
$$

where $a_{1}, a_{2}, a_{3}$ are integers such that $2 \leqslant a_{1} \leqslant a_{2} \leqslant a_{3}$, and $x_{1}, x_{2}, x_{3}$ are coordinates on $\mathbb{A}^{3}$. By [118, Lemma 4], the affine variety $V$ admits an effective $\mathbb{G}_{\mathrm{a}}$-action $\Longleftrightarrow$ $a_{1}=a_{2}=2$.

Affine varieties that do not admit effective $\mathbb{G}_{\mathrm{a}}$-actions are often called rigid $[7,8,24$, 65, 82,118]. Applying [120, Corollary 2.1.4] and [10, Proposition 4.1] to affine cones over projective varieties, we obtain the following result:

Theorem 1.20. Let $V$ be the affine cone in $\mathbb{A}^{n+1}$ over a projectively normal subvariety $X \subset \mathbb{P}^{n}$. Suppose that $V$ is rigid and $\operatorname{Aut}(X)$ is finite. Then there exists an exact sequence of groups

$$
1 \longrightarrow \mathbb{G}_{\mathrm{m}} \longrightarrow \operatorname{Aut}(V) \longrightarrow \operatorname{Aut}(X),
$$

so that $\operatorname{Aut}(V)$ is a finite extension of the torus $\mathbb{G}_{\mathrm{m}}$ by a finite subgroup in $\operatorname{Aut}(X)$.

In particular, combining this result with the negative answer to Question 1.12, we obtain:

Corollary 1.21. If $V$ is the affine hypersurface from Question 1.12, then

$$
\operatorname{Aut}(V)=\mathbb{G}_{\mathrm{m}} \times\left(\mu_{3}^{3} \rtimes \widetilde{S}_{4}\right) .
$$

Both Question 1.12 and Example 1.19 are very special cases of the following old conjecture, which has been confirmed in many cases (see [47] and Remark 3.19).

Conjecture 1.22 ([64,118]). Let $V$ the Pham-Brieskorn hypersurface in $\mathbb{A}^{n}$ with $n \geqslant 3$ given by

$$
x_{1}^{a_{1}}+x_{2}^{a_{2}}+\cdots+x_{n}^{a_{n}}=0,
$$

where $a_{1}, \ldots, a_{n}$ are integers such that $2 \leqslant a_{1} \leqslant \ldots \leqslant a_{n}$, and $x_{0}, x_{1}, \ldots, x_{n}$ are coordinates on $\mathbb{A}^{n}$. Suppose that $a_{2} \geqslant 3$. Then the affine hypersurface $V$ is rigid.

In fact, using Theorem 1.15, we can restate Question 1.12 as follows:

Question 1.23. Let $X$ be the Fermat cubic surface. Does $X$ contain $\left(-K_{X}\right)$-polar cylinder?

As we already mentioned, this question has a negative answer. Moreover, we will see later that the answer is also negative for any smooth cubic surface (cf. Theorem 2.8). This brings us to the following problem.

Problem 1.24. Describe Fano varieties that do not contain anticanonical polar cylinders. 
This problem has been solved for del Pezzo surfaces with Du Val singularities in [38, 39, 120]. However, it is still open for smooth Fano threefolds and singular del Pezzo surfaces with quotient singularities. For Fano varieties whose divisor class groups is of rank 1, Problem 1.24 is equivalent to the cylindricity problem (the problem of existence of cylinders).

Remark 1.25. One can consider Problem 1.24 for Fano varieties defined over an arbitrary possibly algebraically non-closed field. In Section 3.3, we will give a motivation for doing this.

Let us present one obstruction for the existence of anticanonical polar cylinders in Fano varieties. Recall from [29,205] that the $\alpha$-invariant of Tian of the Fano variety $X$ is the number

$$
\alpha(X)=\sup \left\{\begin{array}{l|l}
\lambda \in \mathbb{Q} & \begin{array}{l}
\text { the log pair }(X, \lambda D) \text { is log canonical } \\
\text { for any effective } \mathbb{Q} \text {-divisor } D \sim_{\mathbb{Q}}-K_{X}
\end{array}
\end{array}\right\} .
$$

This number plays an important role in K-stability of Fano varieties, since $X$ is K-stable if

$$
\alpha(X)>\frac{\operatorname{dim}(X)}{\operatorname{dim}(X)+1} .
$$

For K-stability, see the survey article [213] in this volume. On the other hand, we have the following result.

Theorem 1.26. Let $X$ be a Fano variety that has at most Kawamata log terminal singularities. If $\alpha(X) \geqslant 1$, then $X$ does not contain $\left(-K_{X}\right)$-polar cylinders.

Proof. Suppose $X$ contains a $\left(-K_{X}\right)$-polar cylinder. Then $U \cong Z \times \mathbb{A}^{1}$ for an affine variety $Z$, and

$$
U=X \backslash \operatorname{Supp}(D)
$$

for some effective $\mathbb{Q}$-divisor $D$ on $X$ such that $D \sim \mathbb{Q}-K_{X}$. Arguing as in the proof Corollary 2.7, we see that the $\log$ pair $(X, D)$ is not $\log$ canonical in this case, so that $\alpha(X)<1$.

Let us show how to use this obstruction.

Example 1.27. Let $X$ be a del Pezzo surface with Du Val singularities of degree $K_{X}^{2}=1$ such that one of the following two conditions holds:

(1) either $X$ has 2 singular points of type $A_{3}$ and 2 singular points of type $A_{1}$;

(2) or the surface $X$ has 4 singular points of type $\mathrm{A}_{2}$.

By [214, Theorem 1.2], the surface $X$ exists, and it is uniquely determined by its singularities. Moreover, it follows from [214, Table 4.1] that the pencil $\left|-K_{X}\right|$ contains exactly 4 singular fibers. They are singular fibers of types $I_{4}$ and $I_{2}$ (in the first case) or of types $I_{2}$ (in the second case). This gives $\alpha(X)=1$ by [34, Theorem 1.25], so that $X$ contains no anticanonical polar cylinders. Since the group $\mathrm{Cl}(X)$ is of rank 1 , the surface $X$ contains no cylinders at all. 
Remark 1.28. Implicitly, Theorem 1.26 has been already used by many people for quite some time. For instance, Miyanishi conjectured in [93] that the smooth locus of a del Pezzo surface with quotient singularities and Picard rank 1 admits a finite unramified covering that contains a cylinder. It turned out to be wrong. Namely, in [119, Example 21.3.3], Keel and McKernan have constructed a singular del Pezzo surface $X$ with quotient singularities such that $\rho(X)=1$ and $\alpha(X) \geqslant 1$, but its smooth locus has trivial algebraic fundamental group. Thus, its smooth locus does not admit non-trivial unramified coverings, and $X$ does not contain cylinders by Theorem 1.26.

Using Theorem 1.26, we can create many rational Fano varieties without anticanonical polar cylinders. Indeed, if $X$ and $Y$ are Fano varieties that have Kawamata log terminal singularities, then it follows from [29, Lemma 2.29] and [133, Proposition 8.11] that

$$
\alpha(X \times Y)=\min \{\alpha(X), \alpha(Y)\} .
$$

Thus, if $S$ is a general smooth del Pezzo surface with $K_{S}^{2}=1$, then $\alpha(S)=1$ by [31, Theorem 1.7], which implies that we also have $\alpha(X)=1$ for the $2 n$-dimensional smooth Fano variety

$$
X=\underbrace{S \times S \times \cdots \times S}_{n \text { times }},
$$

so that $X$ does not contain $\left(-K_{X}\right)$-polar cylinders, but $X$ is cylindrical, because $S$ is cylindrical. We can construct many similar examples using [34, 36, 37, 43, 195].

Example 1.29. Let $S$ be a general smooth del Pezzo surface with $K_{S}^{2}=1$, and let $Y$ be a general smooth hypersurface in $\mathbb{P}\left(1^{n+1}, n\right)$ of degree $2 n$ for $n \geqslant 3$. Then $\alpha(S)=1$ by [31, Theorem 1.7], and $\alpha(Y)=1$ by [195, Theorem 2] (see also [37]). Let $X=S \times Y$. Then $\operatorname{dim}(X)=2+n \geqslant 5$ and

$$
\alpha(X)=\min \{\alpha(S), \alpha(Y)\}=1,
$$

so that $X$ contains no $\left(-K_{X}\right)$-polar cylinder by Theorem 1.26. But $X$ is cylindrical.

Surprisingly, we do not know a single example of a cylindrical smooth Fano threefold that contains no anticanonical polar cylinder (cf. Examples 3.14, 3.15, 3.16 and 3.17).

Problem 1.30. Find a cylindrical smooth Fano threefold without anticanonical polar cylinder.

Note that there are Fano varieties without cylinders whose $\alpha$-invariant of Tian is smaller than 1. For instance, if $X$ is the del Pezzo surface from Example 1.2, then $\alpha(X)=$ $\frac{1}{2}$ by [34, Theorem 1.25]. On the other hand, this surface does not contain cylinders [39]. Note that it is K-polystable [161]. Surprisingly, all known K-unstable Fano varieties are also cylindrical.

Example 1.31 ([68-70,113]). Let $X$ be a smooth Fano variety of dimension $n \geqslant 2$ such that

$$
-K_{X} \sim(n-1) H
$$


where $H$ is an ample divisor such that $H^{n}=5$. Then $n \in\{2,3,4,5,6\}$, and $X$ is unique for each $n$. The divisor $H$ is very ample, and the linear system $|H|$ gives an embedding $X \hookrightarrow \mathbb{P}^{n+3}$ such that the image is a section of the Grassmannian $\operatorname{Gr}(2,5) \subset \mathbb{P}^{9}$ by a linear subspace of dimension $3+n$. Moreover, if $n \neq 2$, then $\operatorname{Pic}(X)=\mathbb{Z}[H]$. Furthermore, the following assertions hold.

- The variety $X$ contains a Zariski open subset isomorphic to $\mathbb{A}^{n}$, so that it is cylindrical. If $n \neq 5$, this follows from Example 1.1 and Theorems 3.6 and 3.20 (see also $[69,186]$ ). If $n=5$, then $X$ contains a plane $\Pi$ such that there exists the following Sarkisov link:

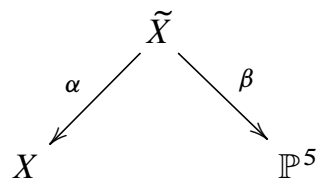

where $\alpha$ is the blowup of the plane $\Pi$, and $\beta$ is the blowup of a smooth cubic scroll in $\mathbb{P}^{5}$. This easily implies that $X$ contains a Zariski open subset isomorphic to $\mathbb{A}^{5}$.

- If $n \in\{2,3,6\}$, then $X$ is known to be K-polystable (see, for example, [30, 31, 169, 206,216]). On the other hand, if $n \in\{4,5\}$, then $X$ is K-unstable by [67].

Keeping in mind Theorem 1.26 and examples of K-stable Fano varieties without anticanonical polar cylinders (for example, smooth del Pezzo surfaces of degree 1, 2 and 3), we pose:

Conjecture 1.32. Let $X$ be a Fano variety that has at most Kawamata log terminal singularities. If $X$ does not contain $\left(-K_{X}\right)$-polar cylinders, then $X$ is $K$-polystable.

For a projective variety $X$, consider the following subset of the cone of ample $\mathbb{Q}$-divisors on $X$ :

$$
\operatorname{Amp}^{\text {cyl }}(X)=\{H \in \operatorname{Amp}(X) \mid \text { there is an } H \text {-polar cylinder on } X\} .
$$

Let us call it the cone of cylindrical ample divisors of the variety $X$. We have seen in Examples 1.2 that $\operatorname{Amp}^{\text {cyl }}(X)$ can be empty even if $X$ is a Fano variety. Thus, we can enhance Problem 1.24:

Problem 1.33. For a given Fano variety $X$, describe the cone $\operatorname{Amp}^{\mathrm{cyl}}(X)$.

This problem is not yet solved even for smooth del Pezzo surfaces. However, we know the answer for many of them (see [40]). Namely, if $X$ is a smooth del Pezzo surface such that $K_{X}^{2} \geqslant 4$, then

$$
\operatorname{Amp}^{\mathrm{cyl}}(X)=\operatorname{Amp}(X) .
$$

On the other hand, if $K_{X}^{2} \leqslant 3$, then $-K_{X} \notin \operatorname{Amp}^{\text {cyl }}(X)$. This gives an evidence for:

Conjecture 1.34. If $X$ is a Fano variety, then

$$
-K_{X} \in \operatorname{Amp}^{\text {cyl }}(X) \Longleftrightarrow \operatorname{Amp}^{\text {cyl }}(X)=\operatorname{Amp}(X) .
$$


Let us describe the structure of this survey. In Section 2 we review results about polar cylinders in rational surfaces. In Section 3, we describe results about cylinders in smooth Fano threefolds, smooth Fano fourfolds, and del Pezzo fibrations. In Section 4, we survey results on three topics that are closely related to the main topic of this survey: flexibility of affine varieties with a special accent on the flexibility of affine cones over Fano varieties, cylinders in the complements to hypersurfaces in weighted projective spaces, and compactifications of $\mathbb{C}^{n}$. Finally, in Appendix A, we present some results about singularities of two-dimensional log pairs, which are used in Section 2 to prove the absence of polar cylinders in some del Pezzo surfaces.

\section{Notations}

Throughout this paper, we will use the following notation:

- $\mu_{n}$ is a cyclic subgroup of order $n$;

- $\mathbb{G}_{\mathrm{a}}$ is a one-dimensional unipotent additive group;

- $\mathbb{G}_{\mathrm{m}}$ is a one-dimensional algebraic torus;

- $\mathbb{F}_{n}$ is the Hirzebruch surface;

- $\quad \mathbb{P}^{n}$ is the $n$-dimensional projective space over $\mathbb{k}$;

- $\quad \mathbb{A}^{n}$ is the $n$-dimensional affine space over $\mathbb{k}$;

- $\mathbb{P}\left(a_{1}, \ldots, a_{n}\right)$ is the weighted projective space;

- for a variety $X$, we denote by $\rho(X)$ the rank of its Picard group.

\section{Cylinders in del Pezzo surfaces}

In this section, we review results about cylinders in del Pezzo surfaces. A del Pezzo surface means here a two-dimensional Fano variety with at most quotient singularities. Recall that a smooth del Pezzo surface is either $\mathbb{P}^{1} \times \mathbb{P}^{1}$, or a blowup of $\mathbb{P}^{2}$ in at most 8 points such that

- at most 2 points are contained in a line;

- at most 5 points are contained in a conic;

- there is no singular cubic in $\mathbb{P}^{2}$ that contains 8 points and is singular in one of them.

A Gorenstein del Pezzo surface is a del Pezzo surface whose anticanonical divisor is Cartier, equivalently a del Pezzo surface with only Du Val singularities. Such surface is either a quadric, or its minimal resolution of singularities can be obtained by blowing up $\mathbb{P}^{2}$ in at most 8 points such that at most 3 of them are contained in a line, and at most 6 of them are contained in a conic.

First, let us go over basic facts about cylinders in rational surfaces. 


\subsection{Cylinders in rational surfaces}

Observe that every smooth rational surface is cylindrical. This immediately follows from the fact that $\mathbb{P}^{2}$ contains a cylinder and the following:

Lemma 2.1. Let $C$ be an irreducible curve in $\mathbb{F}_{n}$ that is a section of the natural projection $\mathbb{F}_{n} \rightarrow \mathbb{P}^{1}$, and let $F_{1}, \ldots, F_{r}$ be fibers of this projection, where $r \geqslant 1$. Then

$$
\mathbb{F}_{n} \backslash\left(C \cup F_{1} \cup \cdots \cup F_{r}\right)
$$

is a cylinder.

Proof. Performing appropriate elementary birational transformations, we may assume that $C^{2}=0$, so that $n=0$. In this case, the required assertion is obvious.

However, as we have seen already in Example 1.2, there are singular rational surfaces that contain no cylinders. Let us explain how to find many such rational surfaces and provide an obstruction for the existence of cylinders (see Remark 2.3 below), which will be used in Section 2.2 to show the absence of anticanonical polar cylinders in smooth del Pezzo surfaces of degree 1, 2 and 3.

Let $S$ be a rational surface with quotient singularities and suppose that $S$ contains a cylinder $U$. Then $U$ is a Zariski open subset in $S$ such that $U \cong \mathbb{A}^{1} \times Z$ for some affine curve $Z$. We then have the following commutative diagram

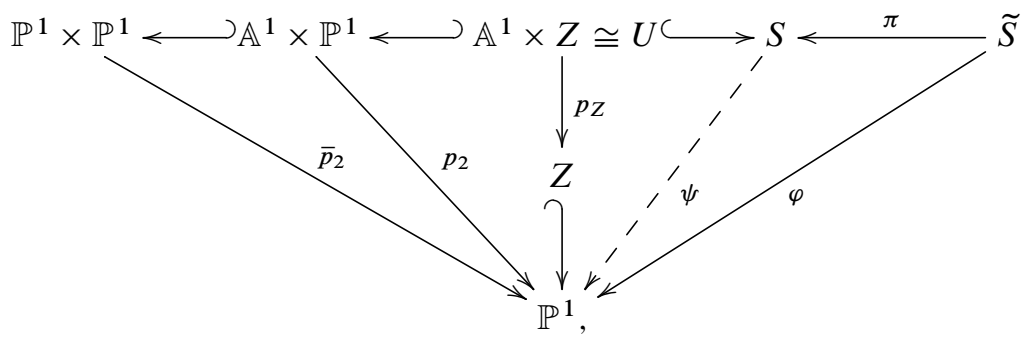

where $p_{Z}, p_{2}$ and $\bar{p}_{2}$ are the natural projections to the second factors, $\psi$ is the rational map induced by $p_{Z}, \pi$ is a birational morphism resolving the indeterminacy of $\psi$ and $\varphi$ is a morphism. By construction, a general fiber of $\varphi$ is $\mathbb{P}^{1}$. Let $C_{1}, \ldots, C_{n}$ be the irreducible curves in $S$ such that

$$
S \backslash U=\bigcup_{i=1}^{n} C_{i} .
$$

The curves $C_{1}, \ldots, C_{n}$ generate the divisor class group $\mathrm{Cl}(S)$ of the surface $S$, because $\mathrm{Cl}(U)=0$. In particular, one has

$$
\operatorname{rank} \mathrm{Cl}(S) \leqslant n
$$

Let $E_{1}, \ldots, E_{r}$ all be exceptional curves of the morphism $\pi$ (if any), and let

$$
\Gamma=\mathbb{P}^{1} \times \mathbb{P}^{1} \backslash \mathbb{A}^{1} \times \mathbb{P}^{1} .
$$


Denote by $\widetilde{C}_{1}, \ldots, \widetilde{C}_{n}$ and $\widetilde{\Gamma}$ the proper transforms $\widetilde{S}$ of the curves $C_{1}, \ldots, C_{n}$ and $\Gamma$, respectively. Then $\widetilde{\Gamma}$ is a section of the conic bundle $\varphi$, and $\widetilde{\Gamma}$ is one of the curves $\widetilde{C}_{1}, \ldots, \widetilde{C}_{n}$ and $E_{1}, \ldots, E_{r}$. Moreover, all other curves among $\widetilde{C}_{1}, \ldots, \widetilde{C}_{n}$ and $E_{1}, \ldots, E_{r}$ are components of some fibers of $\varphi$. Thus, we may assume that either $\widetilde{\Gamma}=\widetilde{C}_{1}$ or $\widetilde{\Gamma}=E_{r}$. Then $\psi$ is a morphism $\Longleftrightarrow \widetilde{\Gamma}=\widetilde{C}_{1}$.

Let $\lambda_{1}, \ldots, \lambda_{n}$ be arbitrary rational numbers, and let $D=\lambda_{1} C_{1}+\cdots+\lambda_{n} C_{n}$. Then

$$
K_{\tilde{S}}+\sum_{i=1}^{n} \lambda_{i} \widetilde{C}_{i}+\sum_{i=1}^{r} \mu_{i} E_{i} \sim_{\mathbb{Q}} \pi^{*}\left(K_{S}+D\right)
$$

for some real numbers $\mu_{1}, \ldots, \mu_{r}$. Let $\widetilde{F}$ be a general fiber of $\varphi$. Then $K_{\tilde{S}} \cdot \widetilde{F}=-2$ by the adjunction formula. Put $F=\pi(\widetilde{F})$. If $\widetilde{\Gamma}=E_{r}$, then

$$
-2+\mu_{r}=\left(K_{\tilde{S}}+\sum_{i=1}^{n} \lambda_{i} \widetilde{C}_{i}+\sum_{i=1}^{r} \mu_{i} E_{i}\right) \cdot \widetilde{F}=\pi^{*}\left(K_{S}+D\right) \cdot \widetilde{F}=\left(K_{S}+D\right) \cdot F .
$$

Similarly, if $\widetilde{\Gamma}=C_{1}$, then

$$
-2+\lambda_{1}=\left(K_{\tilde{S}}+\sum_{i=1}^{n} \lambda_{i} \widetilde{C}_{i}+\sum_{i=1}^{r} \mu_{i} E_{i}\right) \cdot \widetilde{F}=\pi^{*}\left(K_{S}+D\right) \cdot \widetilde{F}=\left(K_{S}+D\right) \cdot F .
$$

On the other hand, if $K_{S}+D$ is pseudo-effective, then $\left(K_{S}+D\right) \cdot F \geqslant 0$.

Remark 2.3. We are therefore able to draw the following conclusions:

- if $K_{S}+D$ is pseudo-effective, then $(S, D)$ is not log canonical;

- if $K_{S}+D$ is pseudo-effective and $\lambda_{i}<2$ for each $i \in\{1, \ldots, n\}$, then $\psi$ is not a morphism.

Corollary 2.4. A rational surface with quotient singularities and pseudo-effective canonical divisor cannot contain any cylinder.

Now we present two examples of rational singular surfaces with nef canonical divisors, which do not contain cylinders by Corollary 2.4. For more examples, see [103, 142, 143, $163,164,166,167,211]$.

Example 2.5 (cf. [162]). Let $E$ be the Fermat cubic curve in $\mathbb{P}^{2}$. Take $\sigma \in \operatorname{Aut}(E)$ of order 6 that fixes a point in $E$. Let $S=E \times E /\langle\sigma\rangle$, where $\sigma$ acts on $E \times E$ diagonally. Then $S$ is rational. Moreover, it has quotient singularities and $6 K_{S} \sim 0$. Then $S$ contains no cylinder by Corollary 2.4 .

Example 2.6 ([129]). Let $a_{0}, a_{1}, a_{2}, a_{3}, w_{0}, w_{1}, w_{2}, w_{3}$ be positive integers such that

- $a_{0} \geqslant 4, a_{1} \geqslant 4, a_{2} \geqslant 4, a_{3} \geqslant 4$;

- $a_{0} w_{0}+w_{1}=a_{1} w_{1}+w_{2}=a_{2} w_{2}+w_{3}=a_{3} w_{3}+w_{0}$;

- $\operatorname{gcd}\left(w_{0}, w_{2}\right)=1, \operatorname{gcd}\left(w_{1}, w_{3}\right)=1$. 
From the first condition above we obtain

$$
\left\{\begin{array}{l}
w_{0}=a_{1} a_{2} a_{3}-a_{2} a_{3}+a_{3}-1, \\
w_{1}=a_{0} a_{2} a_{3}-a_{0} a_{3}+a_{0}-1, \\
w_{2}=a_{0} a_{1} a_{3}-a_{0} a_{1}+a_{1}-1, \\
w_{3}=a_{0} a_{1} a_{2}-a_{1} a_{2}+a_{2}-1 .
\end{array}\right.
$$

Let $S$ be the hypersurface in $\mathbb{P}\left(w_{0}, w_{1}, w_{2}, w_{3}\right)$ defined by the following equation:

$$
x_{0}^{a_{0}} x_{1}+x_{1}^{a_{1}} x_{2}+x_{2}^{a_{2}} x_{3}+x_{3}^{a_{3}} x_{0}=0,
$$

where $x_{0}, x_{1}, x_{2}$ and $x_{3}$ are coordinates of weights $w_{0}, w_{1}, w_{2}, w_{3}$, respectively. Then

$$
K_{S}=\mathcal{O}_{S}\left(a_{0} a_{1} a_{2} a_{3}-w_{0}-w_{1}-w_{2}-w_{3}-1\right)
$$

and $a_{0} a_{1} a_{2} a_{3}-w_{0}-w_{1}-w_{2}-w_{3}-1>0$, so that $K_{S}$ is ample. But $S$ is rational by [129, Theorem 39]. By Corollary 2.4, the surface $S$ cannot contain any cylinder.

We are mostly interested in cylinders in del Pezzo surfaces. Applying our Remark 2.3 to them, we obtain the following special case of Theorem 1.26, which we already applied in Example 1.27.

Corollary 2.7. Suppose that $-K_{S}$ is ample, and $U$ is a $\left(-K_{S}\right)$-polar cylinder. Then

$$
\alpha(S)<1 \text {. }
$$

Proof. There exists an effective $\mathbb{Q}$-divisor $D^{\prime}$ on the surface $S$ such that $D^{\prime} \sim \mathbb{Q}-K_{S}$ and

$$
D^{\prime}=\sum_{i=1}^{n} a_{i} C_{i},
$$

for some positive rational numbers $a_{1}, \ldots, a_{n}$. Let $D=D^{\prime}$. Then $K_{S}+D \sim_{\mathbb{Q}} 0$ is pseudo-effective, so that $(S, D)$ is not $\log$ canonical by Remark 2.3, which implies that $\alpha(S)<1$.

Now, we state main result of this section, which implies negative answer to Question 1.12.

Theorem 2.8 ([38, 39, 120, 123]). Let $S$ be a del Pezzo surface that has at most Du Val singularities. Then $S$ does not contain $\left(-K_{S}\right)$-polar cylinders exactly when:

- $K_{S}^{2}=1$ and $S$ allows at most singular points of types $\mathrm{A}_{1}, \mathrm{~A}_{2}, \mathrm{~A}_{3}, \mathrm{D}_{4}$ if any;

- $K_{S}^{2}=2$ and $S$ allows at most singular points of type $\mathrm{A}_{1}$ if any;

- $K_{S}^{2}=3$ and $S$ is smooth.

Corollary 2.9. A smooth del Pezzo surface $S$ contains a $\left(-K_{S}\right)$-polar cylinder $\Longleftrightarrow$ $K_{S}^{2} \geqslant 4$. 
In the next two subsections, we will explain how to prove Theorem 2.8. Now let us use this result to find all del Pezzo surfaces with Du Val singularities that contain no cylinder.

Theorem 2.10 ([17, Theorem 1.6]). Let $S$ be a del Pezzo surface that has Du Val singularities. Then $S$ contains no cylinder $\Longleftrightarrow$ it is one of the surfaces described in Examples 1.2 and 1.27 .

Proof. If $S$ is one of the surfaces from Examples 1.2 and 1.27, then $\rho(S)=1$, so that it does not contain cylinders by Theorem 2.8. To prove the converse assume that $S$ contains no cylinder. Let us show that $S$ is one of the singular del Pezzo surfaces described in Examples 1.2 and 1.27. If $\rho(S)=1$, this follows from Theorem 2.8 and [214, Theorem 1.2].

We may assume that $\rho(S) \geqslant 2$. Let us seek for a contradiction. Since every smooth rational surface contains a cylinder, we see that $S$ is singular. Then $K_{S}^{2} \leqslant 2$ by Theorem 2.8.

Let $\pi: S \rightarrow Y$ be the contraction of an extremal ray of the Mori cone $\overline{\mathrm{NE}}(S)$ of the surface $S$. Then it follows from [157] that one of the following cases hold:

- $\quad$ either $\pi$ is a conic bundle, $Y=\mathbb{P}^{1}$ and $\rho(S)=2$;

- $\quad$ or $\pi$ is birational, $Y$ is a del Pezzo surface with Du Val singularities, $\rho(Y)=\rho(S)+1$; the morphism $\pi$ is a weighted blowup of a smooth point in $Y$ with weights $(1, k)$ for $k \geqslant 1$, and $K_{Y}^{2}=K_{S}^{2}+k$.

Suppose that $\pi$ is a conic bundle. Then we have the following commutative diagram:

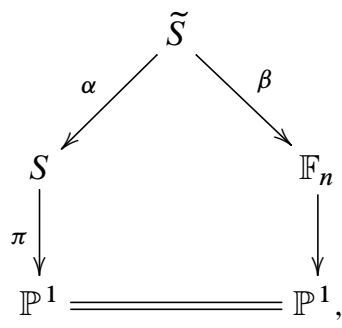

where $\alpha$ is a minimal resolution of singularities, $\beta$ is a birational map, and $\mathbb{F}_{n} \rightarrow \mathbb{P}^{1}$ is a natural projection. On the other hand, it follows from Tsen's theorem that $S$ contains a smooth irreducible curve $Z$ that is a section of the conic bundle $\pi$. Let $C$ be its proper transform on $\mathbb{F}_{n}$. Then

$$
S \backslash\left(Z \cup T_{1} \cup \cdots \cup T_{r}\right) \cong S \backslash\left(C \cup F_{1} \cup \cdots \cup F_{r}\right),
$$

where $T_{1}, \ldots, T_{r}$ are fibers of $\pi$ that contain singular points of the surface $S$, and $F_{1}, \ldots, F_{r}$ are fibers of the projection $\mathbb{F}_{n} \rightarrow \mathbb{P}^{1}$ over the points $\pi\left(T_{1}\right), \ldots, \pi\left(T_{r}\right)$, respectively. Then $S$ contains a cylinder by Lemma 2.1, which is a contradiction.

We see that $\pi$ is birational. Let $E$ be the $\pi$-exceptional curve. If $Y$ contains a cylin$\operatorname{der} U$, then it also contains a cylinder $U^{\prime} \subset U$ such that $\pi(E) \notin U^{\prime}$, so that its preimage 
in $S$ is a cylinder as well. Thus, the surface $Y$ does not contain cylinders. Then $Y$ is singular and $K_{Y}^{2} \leqslant 2$ by Theorem 2.8 .

We see that $K_{Y}^{2}=2$ and $\pi$ is a blowup of a smooth point in $Y$. If $\rho(Y) \geqslant 2$, then we can apply the same arguments to $Y$ to show that it contains a cylinder. Hence, we conclude that $\rho(Y)=1$. On the other hand, all singularities of the surface $Y$ are ordinary double points by Theorem 2.8. We see that $K_{Y}^{2}=2$ and $Y$ has 7 singular points of type $\mathrm{A}_{1}$. But such a surface does not exist.

Let us conclude this subsection by presenting few results about polar cylinders in arbitrary rational surfaces. To do this, fix an ample $\mathbb{Q}$-divisor $H$ on the surface $S$. If $S$ contains an $H$-polar cylinder, we say that $H$ is cylindrical. The cylindrical ample $\mathbb{Q}$-divisors on $S$ form a cone, which we denoted earlier by $\operatorname{Amp}^{\text {cyl }}(S)$. To investigate this cone, consider the following number:

$$
\mu_{H}=\inf \left\{\lambda \in \mathbb{R}_{>0} \mid \text { the } \mathbb{R} \text {-divisor } K_{S}+\lambda H \text { is pseudo-effective }\right\} .
$$

Remark 2.11. The number $\mu_{H}$ is known as the Fujita invariant of the divisor $H$, because it was implicitly used by Fujita in [71-74]. It plays an essential role in Manin's conjecture (see $[15,98])$.

Let $\Delta_{H}$ be the smallest extremal face of the Mori cone $\overline{\mathbb{N E}}(S)$ that contains the divisor $K_{S}+\mu_{H} H$. Put $r_{H}=\operatorname{dim}\left(\Delta_{H}\right)$. Observe that $r_{H}=0$ if and only if $S$ is a del Pezzo surface and $\mu_{H} H \sim \mathbb{Q}-K_{S}$.

Theorem 2.12 ([45]). Suppose that $S$ is smooth, $r_{H}+K_{S}^{2} \leqslant 3$, and the self-intersection of every smooth rational curve in $S$ is at least -1 . Then $S$ does not contain $H$-polar cylinders.

Note that if $S$ is smooth del Pezzo surface, then the self-intersection of every smooth rational curve in $S$ is at least -1 . Moreover, it follows from [52, Proposition 2.4] that this condition also holds if $S$ is obtained by blowing up $\mathbb{P}^{2}$ at any number of points in general position.

Corollary 2.13 ([40]). If $S$ is a smooth del Pezzo surface and $r_{H}+K_{S}^{2} \leqslant 3$, then $H \notin$ $\operatorname{Amp}^{\mathrm{cyl}}(S)$.

On the other hand, we have the following complementary result:

Theorem 2.14 ([40,146]). Suppose that $S$ is a smooth rational surface. If $K_{S}^{2} \geqslant 4$, then

$$
\operatorname{Amp}^{\mathrm{cyl}}(S)=\operatorname{Amp}(S) .
$$

If $K_{S}^{2}=3$ and $-K_{S}$ is not ample, then $\operatorname{Amp}^{\text {cyl }}(S)=\operatorname{Amp}(S)$. If $K_{S}^{2}=3$ and $-K_{S}$ is ample, then

$$
\operatorname{Amp}^{\text {cyl }}(S)=\operatorname{Amp}(S) \backslash \mathbb{Q}>0\left[-K_{S}\right] .
$$

If $S$ is a smooth rational surface and $K_{S}^{2} \leqslant 2$, then $\operatorname{Amp}^{\text {cyl }}(S)$ is poorly understood (see [40]). 


\subsection{Absence of polar cylinders}

Now, we show that smooth del Pezzo surfaces of degree $\leqslant 3$ does not contain any anticanonical polar cylinders, which is one way implication of Corollary 2.9. For singular del Pezzo surfaces of degree $\leqslant 2$ with types of singular points listed in Theorem 2.8, the same implication can be verified in a similar way (see [39] for the details).

Let $S$ be a smooth del Pezzo surface of degree $K_{S}^{2}=d \leqslant 3$, and let $D$ be an effective $\mathbb{Q}$-divisor on the surface $S$, i.e., we have

$$
D=\sum_{i=1}^{r} a_{i} C_{i}
$$

where every $C_{i}$ is an irreducible curve on $S$, and every $a_{i}$ is a non-negative rational number. Suppose that $D \sim \mathbb{Q}-K_{S}$. If $d \in\{2,3\}$, then each $a_{i}$ does not exceed 1 by Lemmas A.9 and A.10. Similarly, if $d=1$, we have

$$
1=d=K_{S}^{2}=D \cdot\left(-K_{S}\right)=\sum_{i=1}^{r} a_{i} C_{i} \cdot\left(-K_{S}\right) \geqslant a_{i} C_{i} \cdot\left(-K_{S}\right),
$$

which immediately implies that $a_{i} \leqslant 1$ for each $i$.

Theorem 2.15. Let $P$ be a point in $S$. Suppose that the log pair $(S, D)$ is not log canonical at $P$. Then there exists a curve $T \in\left|-K_{S}\right|$ such that

- the curve $T$ is singular at $P$;

- the log pair $(S, T)$ is not log canonical at $P$;

- $\operatorname{Supp}(T) \subseteq \operatorname{Supp}(D)$.

Proof. We consider the cases $d=1, d=2$, and $d=3$, separately. See the proof of [38, Theorem 1.12] for an alternative proof in the case $d=3$.

Suppose that $K_{S}^{2}=1$. Let $C$ be a curve in $\left|-K_{S}\right|$ that passes through $P$. Then $C$ is irreducible. If $C$ is not contained in the support of $D$, then it follows from Lemma A.3 that

$$
1=d \geqslant K_{S}^{2}=D \cdot C \geqslant \operatorname{mult}_{P}(D)>1 .
$$

This shows that $C \subset \operatorname{Supp}(D)$. If $(S, C)$ is not $\log$ canonical at $P$, then we can put $T=C$ and we are done. Thus, we may assume that $(S, C)$ is $\log$ canonical at $P$. Then Remark A.2 implies the existence of an effective $\mathbb{Q}$-divisor $D^{\prime}$ such that $D^{\prime} \sim \mathbb{Q}-K_{S}$, the curve $C$ is not contained in the support of $D^{\prime}$, and $\left(S, D^{\prime}\right)$ is not $\log$ canonical at $P$. Now Lemma A.3 implies that

$$
1=d \geqslant K_{S}^{2}=D^{\prime} \cdot C \geqslant \operatorname{mult}_{P}\left(D^{\prime}\right)>1,
$$

which is absurd.

Now, we suppose that $K_{S}^{2}=2$. In this case there exists a double cover $\tau: S \rightarrow \mathbb{P}^{2}$ branched over a smooth quartic curve $C$. Moreover, we have

$$
D \sim \mathbb{Q}-K_{S} \sim \tau^{*}(L),
$$


where $L$ is a line in $\mathbb{P}^{2}$. By Lemma A.10, we have $\tau(P) \in C$. Now let us choose $L$ to be the tangent line to $C$ at the point $\tau(P)$, and let $R$ be the curve in $\left|-K_{S}\right|$ such that $\tau(R)=L$. Then $\operatorname{mult}_{P}(R)=2$. If $R$ is irreducible and is not contained in the support of $D$, then Lemma A.3 gives

$$
2=d \geqslant K_{S}^{2}=D \cdot R \geqslant \operatorname{mult}_{P}(D) \operatorname{mult}_{P}(R) \geqslant 2 \operatorname{mult}_{P}(D)>2 .
$$

Note that either $R$ is irreducible or $R$ consists of two (-1)-curves that both pass through $P$. Therefore, if one component of the curve $R$ is not contained in the support of the divisor $D$, then we obtain a contradiction in a similar way by intersecting $D$ with this irreducible component. Thus, we may assume that all irreducible component of the curve $R$ are contained in $\operatorname{Supp}(D)$. Now we can use Remark A.2 as in the case $d=1$ to conclude that $(S, R)$ is not $\log$ canonical at $P$. Hence, we can let $T=R$.

Finally, we suppose that $K_{S}^{2}=3$. Then $S$ is a smooth cubic surface in $\mathbb{P}^{3}$, and $-K_{S}$ is rationally equivalent to its hyperplane section. Let $T_{P}$ be the intersection of the surface $S$ with the hyperplane that is tangent to $S$ at the point $P$. Then $T_{P}$ is a reduced cubic curve that is singular at $P$. If $\left(S, T_{P}\right)$ is not $\log$ canonical at $P$ and $\operatorname{Supp}\left(T_{P}\right) \subseteq \operatorname{Supp}(D)$, we can let $T=T_{P}$ and we are done. Therefore, we may assume that at least one of the following two conditions hold:

(1) the $\log$ pair $\left(S, T_{P}\right)$ is $\log$ canonical at $P$;

(2) $\operatorname{Supp}(D)$ does not contain at least one irreducible components of the curve $T_{P}$.

To obtain a contradiction, we may assume by Remark A.2 that at least one irreducible component of the curve $T_{P}$ is not contained in $\operatorname{Supp}(D)$.

If $L_{P}$ is a line that passes through $P$, then $L_{P} \subseteq \operatorname{Supp}(D)$, since otherwise we would get

$$
1 \geqslant D \cdot L_{P} \geqslant \operatorname{mult}_{P}(D) \operatorname{mult}_{P}\left(L_{P}\right) \geqslant \operatorname{mult}_{P}(D)>1
$$

by Lemma A.3. Thus, we see that $\operatorname{mult}_{P}\left(T_{P}\right)=2$.

Let $f: \widetilde{S} \rightarrow S$ be the blowup of the point $P$, let $E$ be the exceptional curve of the blowup $f$, and let $\widetilde{D}$ be the proper transform on $\widetilde{S}$ of the $\mathbb{Q}$-divisor $D$. Then

$$
\operatorname{mult}_{P}(D)>1
$$

by Lemma A.3. Moreover, if follows from Lemma A.5 that the log pair

$$
\left(\widetilde{S}, \widetilde{D}+\left(\operatorname{mult}_{P}(D)-1\right) E\right)
$$

is not $\log$ canonical at some point $Q \in E$. Moreover, there is a commutative diagram

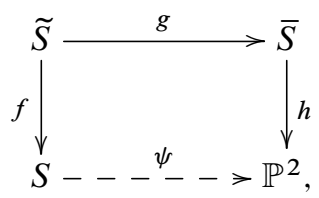


where $\psi$ is a projection from $P$, the morphism $g$ is a contraction of the proper transforms of all lines in $S$ that pass through $P$, and $h$ is a double cover branched over a quartic curve. This quartic curve has at most two ordinary double points, because mult ${ }_{P}\left(T_{P}\right) \neq 3$.

Let $\widetilde{T}_{P}$ be the proper transform on $\widetilde{S}$ of the curve $T_{P}$. Then $Q \in E \cap \widetilde{T}_{P}$ by Lemma A.10. Note that $T_{P}$ is one of the following curves: an irreducible cubic curve, a union of a conic and a line, a union of three lines. Let us consider these cases separately.

Suppose that $T_{P}$ is a union of a conic and a line, so that $T_{P}=L_{P}+C_{P}$, where $L_{P}$ is a line, and $C_{P}$ is an irreducible conic. Then $L_{P} \subset \operatorname{Supp}(D)$, so that $C_{P}$ is not contained in $\operatorname{Supp}(D)$. Thus, we write $D=a L_{P}+\Omega$, where $a \in \mathbb{Q}>0$, and $\Omega$ is an effective $\mathbb{Q}$-divisor on $S$ whose support contains none of the curves $L_{P}$ and $C_{P}$. Put $m=\operatorname{mult}_{P}(\Omega)$. Then $\operatorname{mult}_{P}(D)=m+a$ and

$$
2-2 a=\Omega \cdot C_{P} \geqslant m,
$$

which gives $m+2 a \leqslant 2$. Similarly, we obtain $1+a \geqslant m$ by using

$$
1+a=L_{P} \cdot D=\Omega \cdot L_{P} \geqslant m \text {. }
$$

Denote by $\widetilde{C}_{P}$ the proper transform of the conic $C_{P}$ on the surface $\widetilde{S}$, denote by $\widetilde{L}_{P}$ the proper transform of the line $L_{P}$ on the surface $\widetilde{S}$, and denote by $\widetilde{\Omega}$ the proper transform of the divisor $\Omega$ on the surface $\widetilde{S}$. Put $\tilde{m}=\operatorname{mult}_{Q}(\widetilde{\Omega})$. Then the $\log$ pair

$$
\left(\tilde{S}, a \tilde{L}_{P}+\widetilde{\Omega}+(m+a-1) E\right)
$$

is not $\log$ canonical at $P$. Now, applying Lemma A.3 to this log pair, we obtain

$$
2 a+m+\tilde{m}>2 .
$$

On the other hand, if $Q \in \widetilde{C}_{P}$, then

$$
2-2 a-m=\widetilde{\Omega} \cdot \widetilde{C}_{P} \geqslant \tilde{m},
$$

so that $Q \notin \widetilde{C}_{P}$. Since $Q \in \widetilde{T}_{P}$, we see that $Q \in \widetilde{L}_{P}$. Then we have

$$
1+a-m=\tilde{\Omega} \cdot \widetilde{L}_{P} \geqslant \tilde{m}
$$

so that $2 \geqslant 1+a \geqslant m+\tilde{m} \geqslant 2 \tilde{m}$, which gives $\tilde{m} \leqslant 1$. Thus, we can apply Theorem A.8 to the log pair (2.16) at the point $Q$. This gives

or

$$
\begin{aligned}
m & =\widetilde{\Omega} \cdot E \geqslant(\widetilde{\Omega} \cdot E)_{Q}>2(2-a-m) \\
1+a-m & =\widetilde{\Omega} \cdot \widetilde{L} \geqslant(\widetilde{\Omega} \cdot \widetilde{L})_{Q}>2(1-a),
\end{aligned}
$$

so that we get $3 a+m>3$ or $2 a+m>2$, which is impossible since $a \leqslant 1$ and $m+2 a \leqslant 2$.

Therefore, we conclude that the curve $T_{P}$ a union of three lines. Hence, we have $T_{P}=L_{1}+L_{2}+L_{3}$, where $L_{1}, L_{2}, L_{3}$ are lines in $S$ such that $P=L_{1} \cap L_{2}$ and $P \notin L_{3}$. Then $L_{1} \subset \operatorname{Supp}(D) \supset L_{2}$. Therefore, we can write $D=a_{1} L_{1}+a_{2} L_{2}+\Delta$, 
where $a_{1}$ and $a_{2}$ are some positive rational numbers, and $\Delta$ is an effective $\mathbb{Q}$-divisor whose support does not contain $L_{1}$ and $L_{2}$. Put $\mathbf{m}=\operatorname{mult}_{P}(\Delta)$. Then

$$
\mathbf{m} \leqslant \Delta \cdot L_{1}=\left(H-a_{1} L_{1}-a_{2} L_{2}\right) \cdot L_{1}=1+a_{1}-a_{2},
$$

because $L_{1} \cdot L_{2}=1$ and $L_{1}^{2}=-1$ on the surface $S$. Similarly, we see that

$$
\mathbf{m} \leqslant \Delta \cdot L_{2}=\left(H-a_{1} L_{1}-a_{2} L_{2}\right) \cdot L_{2}=1-a_{1}+a_{2} .
$$

This gives $\mathbf{m} \leqslant 1$. Thus, we can apply Theorem A.8 to the $\log$ pair $(S, D)$ at the point $P$. Then

or

$$
\begin{aligned}
& 1+a_{1}-a_{2}=\Delta \cdot L_{1} \geqslant\left(\Delta \cdot L_{1}\right)_{P}>2\left(1-a_{2}\right) \\
& 1-a_{1}+a_{2}=\Delta \cdot L_{2} \geqslant\left(\Delta \cdot L_{2}\right)_{P}>2\left(1-a_{1}\right),
\end{aligned}
$$

which implies that $a_{1}+a_{2}>1$. On the other hand, we have

$$
0 \leqslant \Delta \cdot L_{3}=\left(H-a_{1} L_{1}-a_{2} L_{2}\right) \cdot L_{3}=1-a_{1}-a_{2},
$$

which implies that $a_{1}+a_{2} \leqslant 1$. The obtained contradiction completes the solution.

We now claim that a smooth del Pezzo surface of degree $d \leqslant 3$ cannot contain a $\left(-K_{S}\right)$ cylinder. If $d \leqslant 2$, the claim is [123, Proposition 5.1]. Similarly, if $d=3$, then the claim is [38, Theorem 1.7]. Let us show how to derive the claim from Theorem 2.15 and Remark 2.3.

Suppose that $S$ contains a $\left(-K_{S}\right)$-polar cylinder $U$. Then

$$
S \backslash U=C_{1} \cup \cdots \cup C_{n}
$$

for some irreducible curves $C_{1}, \ldots, C_{n}$ in $S$, and there are positive rational numbers $\lambda_{1}, \ldots, \lambda_{n}$ such that

$$
\sum_{i=1}^{n} \lambda_{i} C_{i} \sim_{\mathbb{Q}}-K_{S} .
$$

Put $D=\lambda_{1} C_{1}+\cdots+\lambda_{n} C_{n}$. Then $(S, D)$ is not log canonical at some point $P \in S$ by Remark 2.3. Hence, by Theorem 2.15, there exists a curve $T \in\left|-K_{S}\right|$ such that

- the log pair $(S, T)$ is not $\log$ canonical at $P$; and

- $\operatorname{Supp}(T) \subseteq \operatorname{Supp}(D)$.

Then $D \neq T$, because $n>3$ by (2.2), and $T$ does not have more than $d \leqslant 3$ irreducible components. Thus, there exists a rational number $\mu>0$ such that $(1+\mu) D-\mu T$ is effective, and its support does not contain at least one irreducible component of the curve $T$. Then $(S,(1+\mu) D-\mu T)$ is not $\log$ canonical at $P$ by Remark 2.3, which contradicts to Theorem 2.15, since

$$
(1+\mu) D-\mu T \sim \mathbb{Q}-K_{S}
$$




\subsection{Construction of polar cylinders}

Now, we show how to construct anticanonical polar cylinders in singular del Pezzo surfaces with Du Val singularities. We start with the following lemma.

Lemma 2.17 ([120, Theorem 3.19]). Let $S$ be a smooth del Pezzo surface. Suppose that $K_{S}^{2} \geqslant 4$. Then the surface $S$ contains a $\left(-K_{S}\right)$-polar cylinder.

Proof. We may assume that $S \neq \mathbb{P}^{1} \times \mathbb{P}^{1}$. Then there exists a birational map $\sigma: S \rightarrow \mathbb{P}^{2}$ that blows up $k \leqslant 5$ distinct points. Let $E_{1}, \ldots, E_{k}$ be the $\sigma$-exceptional curves, let $C$ be an irreducible conic in $\mathbb{P}^{2}$ that contains all points $\sigma\left(E_{1}\right), \ldots, \sigma\left(E_{k}\right)$, and let $L$ be a line in $\mathbb{P}^{2}$ that is tangent to the conic $C$ at some point that is different from $\sigma\left(E_{1}\right), \ldots, \sigma\left(E_{k}\right)$. Denote by $\widetilde{C}$ and $\widetilde{L}$ the proper transforms on $S$ of the curves $C$ and $L$, respectively. Then

$$
-K_{S} \sim \sigma^{*}\left(-K_{\mathbb{P}^{2}}\right)-\sum_{i=1}^{k} E_{i} \sim_{\mathbb{Q}}(1+\varepsilon) \widetilde{C}+(1-2 \varepsilon) \tilde{L}+\varepsilon \sum_{i=1}^{k} E_{i}
$$

for every positive $\varepsilon<\frac{1}{2}$. On the other hand, we have

$$
S \backslash\left(\widetilde{C} \cup \tilde{L} \cup E_{1} \cup \cdots \cup E_{k}\right) \cong \mathbb{P}^{2} \backslash(C \cup L) \cong\left(\mathbb{A}^{1} \backslash\{0\}\right) \times \mathbb{A}^{1},
$$

so that the surface $S$ contains a $\left(-K_{S}\right)$-polar cylinder.

Now let us present an example of a singular del Pezzo surface of degree 2 that has one singular point of type $\mathrm{A}_{2}$ and contains an anticanonical polar cylinder.

Example 2.18. Let $h: \hat{S} \rightarrow \mathbb{P}^{2}$ be a composition of 10 blowups, let $E_{1}, \ldots, E_{10}$ be the exceptional curves of the birational morphism $h$, let $L_{1}$ and $L_{2}$ be two distinct lines in $\mathbb{P}^{2}$, and let $\hat{L}_{1}$ and $\widehat{L}_{2}$ be their proper transforms on $\hat{S}$, respectively. Now, let us choose $h$ such that the intersections of these twelve curves are depicted as follows:

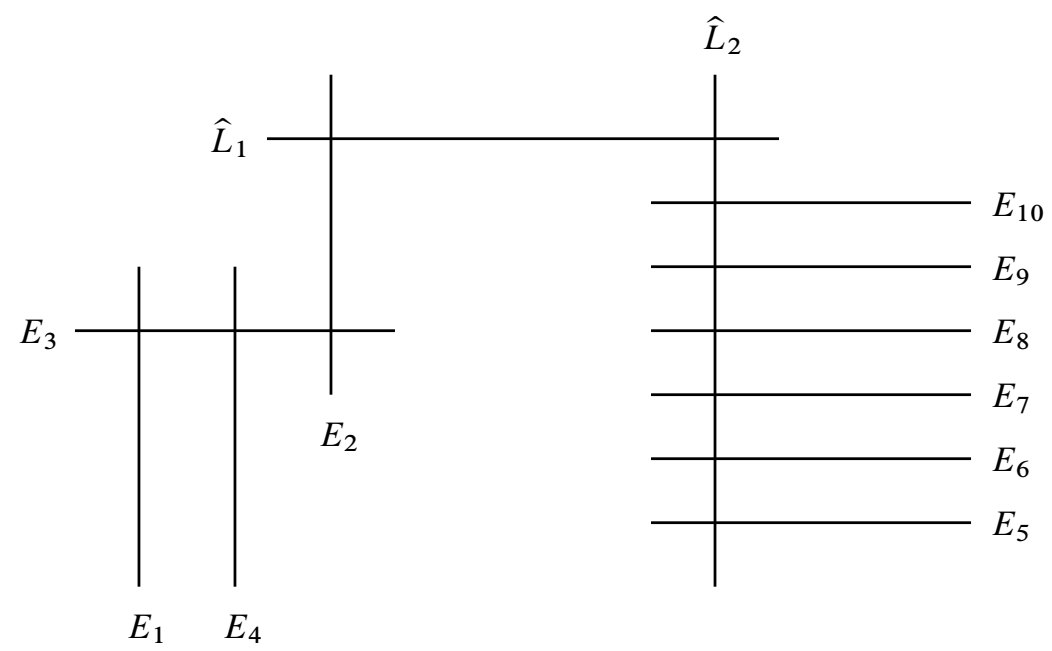


Note that the 10 blowups are arranged in the order indicated by the indices of their exceptional curves $E_{i}$. To describe the intersection form of the curves $\widehat{L}_{1}, \widehat{L}_{2}, E_{1}, \ldots, E_{10}$, observe that

$\widehat{L}_{1}^{2}=-1, \quad \widehat{L}_{2}^{2}=-5, \quad E_{1}^{2}=-3, \quad E_{2}^{2}=-2, \quad E_{3}^{2}=-2, \quad E_{4}^{2}=\cdots=E_{10}^{2}=-1$.

Let $g: \hat{S} \rightarrow \widetilde{S}$ be the contraction of the curves $\widehat{L}_{1}, E_{2}, E_{3}$, and let $\widetilde{L}_{2}, \widetilde{E}_{1}, \widetilde{E}_{4}, \ldots, \widetilde{E}_{10}$ be the proper transforms on $\widetilde{S}$ of the curves $\widehat{L}_{2}, \widehat{E}_{1}, \widehat{E}_{4}, \ldots, \widehat{E}_{10}$, respectively. Then $\widetilde{S}$ is smooth and $K_{\widetilde{S}}^{2}=2$. Moreover, the divisor $-K_{\widetilde{S}}$ is nef. To show this, fix an arbitrary positive rational number $\epsilon<\frac{1}{3}$, let $D_{\widehat{S}}$ be the following $\mathbb{Q}$-divisor:

$$
\begin{aligned}
(2-\epsilon) \hat{L}_{1}+(1+\epsilon) \hat{L}_{2}+(1-\epsilon) E_{1}+(2-2 \epsilon) E_{2}+(2-3 \epsilon) E_{3}+(1-3 \epsilon) E_{4} \\
+\epsilon\left(E_{5}+E_{6}+E_{7}+E_{8}+E_{9}+E_{10}\right)
\end{aligned}
$$

and denote by $D_{\widetilde{S}}$ its proper transform on $\widetilde{S}$. Then $D_{\widehat{S}}$ is effective, $D_{\widehat{S}} \sim_{\mathbb{Q}}-K_{\widehat{S}}$ and $D_{\tilde{S}} \sim \mathbb{Q}-K_{\tilde{S}}$. Moreover, we have $\widetilde{L}_{2}^{2}=\widetilde{E}_{1}=-2, \widetilde{E}_{4}^{2}=0$ and $\widetilde{E}_{5}^{2}=\cdots=\widetilde{E}_{10}^{2}=-1$, so that

$$
-K_{\widetilde{S}} \cdot \widetilde{L}_{2}=-K_{\widetilde{S}} \cdot \widetilde{E}_{1}=0,-K_{\widetilde{S}} \cdot \widetilde{E}_{4}=2,-K_{\widetilde{S}} \cdot \widetilde{E}_{5}=\cdots=-K_{\widetilde{S}} \cdot \widetilde{E}_{10}=1 .
$$

This shows that $-K_{\widetilde{S}}$ is nef. Moreover, we also see that $\widetilde{L}_{2}$ and $\widetilde{E}_{1}$ are the only $(-2)$ curves in $\widetilde{S}$. Let $f: \widetilde{S} \rightarrow S$ be the birational contraction of these two (-2)-curves. Then $S$ is a del Pezzo surface with one singular point of type $\mathrm{A}_{2}$ such that $K_{S}^{2}=2$. Let $D_{S}=$ $f \circ g\left(D_{\widetilde{S}}\right)$. Then $D_{S} \sim_{\mathbb{Q}}-K_{S}$ and

$$
S \backslash \operatorname{Supp}\left(D_{S}\right) \cong \mathbb{P}^{2} \backslash \operatorname{Supp}\left(D_{\mathbb{P}^{2}}\right) \cong \mathbb{A}^{1} \times\left(\mathbb{A}^{1} \backslash\{0\}\right),
$$

so that $S$ contains $\left(-K_{S}\right)$-polar cylinder.

One can use the construction in Example 2.18 to construct an anticanonical polar cylinder in every del Pezzo surface of degree 2 that has a single singular point of type $\mathrm{A}_{2}$ (see [39, §4.3]). Similarly, we can prove the existence part of Theorem 2.8. However, there is an alternative proof, which is more algebraic. Let us describe it following [33].

Let $S$ be a singular del Pezzo surface of degree $K_{S}^{2} \leqslant 3$ that has at most Du Val singularities, and let $P$ be its singular point. Suppose, in addition, that the following conditions hold:

- the singular point $P$ is not of type $\mathrm{A}_{1}$ if $K_{S}^{2}=2$;

- the singular point is not of types $\mathrm{A}_{1}, \mathrm{~A}_{2}, \mathrm{~A}_{3}, \mathrm{D}_{4}$ if $K_{S}^{2}=1$.

Now, let us prove that $S$ contains a $\left(-K_{S}\right)$-polar cylinder (cf. Theorem 2.8 ).

Denote by $\mathbb{P}$ the three-dimensional weighted projective space in which $S$ sits as a hypersurface. Note that $\mathbb{P}=\mathbb{P}^{3}$ (respectively, $\mathbb{P}(1,1,1,2), \mathbb{P}(1,1,2,3)$ ) if $K_{S}^{2}=3$ (respectively, $K_{S}^{2}=2, K_{S}^{2}=1$ ). For the quasi-homogeneous coordinate system for $\mathbb{P}$, we use $[x: y: z: w]$. By a coordinate change, we may assume that $P=[1: 0: 0: 0]$. Then the equation of $S$ can be described as follows: 
- if $K_{S}^{2}=3$, then $S$ is given by

$$
x f_{2}(y, z, w)+f_{3}(y, z, w)=0,
$$

where $f_{2}$ and $f_{3}$ are polynomials of degrees 2 and 3, respectively;

- if $K_{S}^{2}=2$, then $S$ is given by

$$
w^{2}+x\left(a y w+f_{3}(y, z)\right)+f_{4}(y, z)=0,
$$

where $f_{3}$ and $f_{4}$ are polynomials of degrees 3 and 4 , respectively, and $a \in \mathbb{k}$;

- if $K_{S}^{2}=1$, then $S$ is given by

or

$$
\begin{array}{r}
w^{2}+x\left(a y^{2} w+f_{5}(y, z)\right)+f_{6}(y, z)=0 \\
w^{2}+x\left(z w+f_{5}(y, z)\right)+f_{6}(y, z)=0,
\end{array}
$$

where $f_{5}$ and $f_{6}$ are polynomials of degrees 5 and 6 , respectively, and $a \in \mathbb{k}$.

Let $\Pi$ be the hyperplane in $\mathbb{P}$ defined by $x=0$, and let $\pi: S \rightarrow \Pi$ be the map given by

$$
[x: y: z: w]=[0: y: z: w] \text {. }
$$

The hyperplane $\Pi$ is isomorphic to $\mathbb{P}^{2}, \mathbb{P}(1,1,2), \mathbb{P}(1,2,3)$ according to $K_{S}^{2}=3,2,1$, respectively. We denote by $g(y, z, w)$ the coefficient of $x$ in each of equations (2.19), (2.20), (2.21) and (2.22). Namely, if $K_{S}^{2}=3$, then $g(y, z, w)=f_{2}(y, z, w)$. Similarly, if $K_{S}^{2}=2$, then

$$
g(y, z, w)=a y w+f_{3}(y, z) .
$$

Finally, if $K_{S}^{2}=1$, then $g(y, z, w)=z w+f_{5}(y, z)$ or

$$
g(y, z, w)=a y^{2} w+f_{5}(y, z) .
$$

Let $D$ be the divisor on $S$ that is cut out by $g(y, z, w)=0$. If $K_{S}^{2}=3$, then $D$ consists of the lines that contains $P$. There are at most six such lines and they are defined in $\mathbb{P}^{3}$ by

$$
\left\{\begin{array}{l}
g(y, z, w)=0, \\
f_{3}(y, z, w)=0 .
\end{array}\right.
$$

Similarly, if $K_{S}^{2}=2$, then the divisor $D$ consists of at most six curves passing through the point $P$. They are defined in $\mathbb{P}(1,1,1,2)$ by

$$
\left\{\begin{array}{l}
g(y, z, w)=0 \\
w^{2}+f_{4}(y, z)=0 .
\end{array}\right.
$$

Finally, if $K_{S}^{2}=1$, then the divisor $D$ consists of at most five curves passing through the point $P$, which are defined in $\mathbb{P}(1,1,2,3)$ by

$$
\left\{\begin{array}{l}
g(y, z, w)=0, \\
w^{2}+f_{6}(y, z)=0 .
\end{array}\right.
$$


In each case, the number of curves in $D$ is the same as the number of points determined by the corresponding system of equations in $\Pi$. We denote these curves by $L_{1}, \ldots, L_{r}$ in each case. The map $\pi$ contracts each curve $L_{i}$ to a point on $\Pi$.

The equations (2.19), (2.20), (2.21) and (2.22) immediately imply that $\pi$ is a birational map. Moreover, it induces an isomorphism

$$
\tilde{\pi}: S \backslash\left(L_{1} \cup \cdots \cup L_{r}\right) \cong \operatorname{Im}(\tilde{\pi}) \subset \Pi \text {. }
$$

Let $\ell$ be the curve on $\Pi$ defined by $g(y, z, w)=0$. Then $\ell$ can be reducible or nonreduced.

Lemma 2.23. Suppose that $K_{S}^{2}=3$. Then there is a hyperplane section $H$ of the surface $S$ such that the complement $S \backslash\left(H \cup L_{1} \cup \cdots \cup L_{r}\right)$ is a $\left(-K_{S}\right)$-polar cylinder.

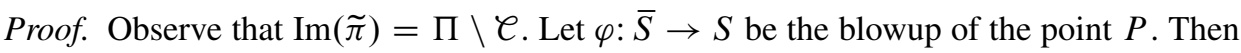
there exists a commutative diagram

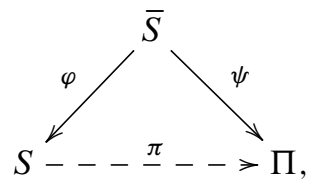

where $\psi$ is the birational morphism that contracts the proper transforms of the lines $L_{1}, \ldots, L_{r}$. Let $E$ be the exceptional curve of the blowup $\varphi$. Then $\psi(E)=\ell$, and $\mathcal{C}$ contains each point $\pi\left(L_{i}\right)$.

If $P$ is an ordinary double point of the cubic surface $S$, then the curve $\ell$ is a smooth conic. Similarly, if $P$ is a singular point of type $\mathrm{A}_{n}$ for $n \geqslant 2$, then $\ell$ splits as a union of two distinct lines. Finally, if $P$ is either of type $\mathrm{D}_{n}$ or of type $\mathrm{E}_{6}$, then $\zeta$ is a double line.

If $\ell$ is smooth, let $\ell$ be a general line in $\Pi$ that is tangent to $\ell$. If $\ell$ is singular, let $\ell$ be a general line in $\Pi$ that passes through a singular point of the conic $\ell$. By a suitable coordinate change, we may assume that $\ell$ is defined by $x=y=0$. Let $H$ be the curve in $S$ cut out by $y=0$. Then

$$
\begin{aligned}
S \backslash\left(H \cup L_{1} \cup \cdots \cup L_{r}\right) & \cong \Pi \backslash(\ell \cup \ell) \\
& \cong\left\{\begin{array}{l}
\left(\mathbb{A}^{1} \backslash\{0,1\}\right) \times \mathbb{A}^{1} \quad \text { if } \ell \text { is a union of two distinct lines, } \\
\left(\mathbb{A}^{1} \backslash\{0\}\right) \times \mathbb{A}^{1} \quad \text { otherwise. }
\end{array}\right.
\end{aligned}
$$

Therefore, $S \backslash\left(H \cup L_{1} \cup \cdots \cup L_{r}\right)$ is a cylinder. But $H+D \sim-3 K_{S}$ and

$$
L_{1} \cup \cdots \cup L_{r}=\operatorname{Supp}(D) .
$$

Thus, the complement $S \backslash\left(H \cup L_{1} \cup \cdots \cup L_{r}\right)$ is a $\left(-K_{S}\right)$-polar cylinder.

To deal with the cases $K_{S}^{2}=1$ and $K_{S}^{2}=2$, let $\ell_{y}$ be the curve in $\mathbb{P}$ that is given by $x=y=0$, and let $H_{y}$ be the curve in the surface $S$ that is cut out by $y=0$. 
Lemma 2.24. If $K_{S}^{2}=2$ or if $K_{S}^{2}=1$ and the surface $S$ is defined by equation (2.21), then the complement $S \backslash\left(H_{y} \cup L_{1} \cup \cdots \cup L_{r}\right)$ is a $\left(-K_{S}\right)$-polar cylinder.

Proof. Observe that the morphism $\tilde{\pi}$ gives an isomorphism

$$
S \backslash\left(H_{y} \cup L_{1} \cup \cdots \cup L_{r}\right) \cong \Pi \backslash\left(\mathcal{C} \cup \ell_{y}\right) .
$$

But $\pi$ maps $S \backslash H_{y}$ onto $\Pi \backslash \ell_{y} \cong \mathbb{A}^{2}$. Thus, if $K_{S}^{2}=2$, then $S \backslash\left(H_{y} \cup L_{1} \cup \cdots \cup L_{r}\right)$ is isomorphic to the complement in $\mathbb{A}^{2}$ of the curve defined by

$$
a w+f_{3}(1, z)=0
$$

Similarly, if $K_{S}^{2}=1$ and $S$ is defined by (2.21), then $S \backslash\left(H_{y} \cup L_{1} \cup \cdots \cup L_{r}\right)$ is isomorphic to the complement in $\mathbb{A}^{2}$ of the curve defined by

$$
a w+f_{5}(1, z)=0
$$

Therefore, in both cases, the complement $S \backslash\left(H_{y} \cup L_{1} \cup \cdots \cup L_{r}\right)$ is a cylinder. Now, arguing as in the proof of Lemma 2.23, we see that $S \backslash\left(H_{y} \cup L_{1} \cup \cdots \cup L_{r}\right)$ is a $\left(-K_{S}\right)$ polar cylinder.

Finally, to deal with the remaining case, let $\ell_{z}$ be the curve in $\mathbb{P}$ that is given by $x=z=0$, and let $H_{z}$ be the hyperplane section of $S$ that is cut by $z=0$.

Lemma 2.25. Suppose that $K_{S}^{2}=1$ and the del Pezzo surface $S$ is given by equation (2.22). Then the complement $S \backslash\left(H_{z} \cup L_{1} \cup \cdots \cup L_{r}\right)$ is a $\left(-K_{S}\right)$-polar cylinder.

Proof. Observe that the morphism $\tilde{\pi}$ gives an isomorphism

$$
S \backslash\left(H_{z} \cup L_{1} \cup \cdots \cup L_{r}\right) \cong \Pi \backslash\left(\mathcal{C} \cup \ell_{z}\right) .
$$

But $\pi$ maps $S \backslash H_{z}$ onto $\Pi \backslash \ell_{z}$. Then $\Pi \backslash\left(\mathcal{U} \cup \ell_{z}\right)$ is the complement of the curve defined by

$$
w+f_{5}(y, 1)=0
$$

in $\Pi \backslash \ell_{z} \cong \mathbb{A}^{2} / \boldsymbol{\mu}_{2}$, where the $\boldsymbol{\mu}_{2}$-action is given by $(y, w) \mapsto(-y,-w)$.

Since $f_{5}(y, 1)$ is an odd polynomial in $y$, the isomorphism $\mathbb{A}^{2} \rightarrow \mathbb{A}^{2}$ defined by

$$
(y, w) \mapsto\left(y, w+f_{5}(y, 1)\right)
$$

is $\mu_{2}$-equivariant and gives an isomorphism between the complement $\Pi \backslash\left(\mathcal{C} \cup \ell_{z}\right)$ and the complement in $\mathbb{A}^{2} / \mu_{2}$ of the image of the curve defined by $w=0$, which is isomorphic to $\mathbb{A}^{1} \backslash\{0\} \times \mathbb{A}^{1}$.

We see that $S \backslash\left(H_{z} \cup L_{1} \cup \cdots \cup L_{r}\right)$ is a cylinder. Now, arguing as in the proof of Lemma 2.23, we conclude that $S \backslash\left(H_{z} \cup L_{1} \cup \cdots \cup L_{r}\right)$ is a $\left(-K_{S}\right)$-polar cylinder. 


\section{Cylinders in higher-dimensional varieties}

In this section, we describe known results about cylinders in smooth Fano threefolds and fourfolds, and varieties fibred into del Pezzo surfaces. Let us say few words about Fano varieties [107, 113, 159].

Let $V$ be a smooth Fano variety of dimension $n \geqslant 3$. The number $\left(-K_{V}\right)^{n}$ is known as the degree of the Fano variety $V$. Put

$$
\iota(V)=\max \left\{t \in \mathbb{N} \mid-K_{V} \sim t H \text { for } H \in \operatorname{Pic}(V)\right\} .
$$

Then $\iota(V)$ is known as the (Fano) index of the variety $V$. It is well known that $1 \leqslant \iota(V) \leqslant$ $n+1$. Moreover, one has

$$
\iota(V)=n+1 \Longleftrightarrow V \cong \mathbb{P}^{n}
$$

Similarly, we have $\iota(V)=n$ if and only if $V$ is a quadric $($ see $[113,125])$.

Remark 3.1 ([68-70,113]). Suppose that $\iota(V)=n-1$. Then

$$
-K_{V} \sim(n-1) H
$$

for some ample divisor $H \in \operatorname{Pic}(V)$. In this case, the variety $V$ is usually called a del Pezzo variety. If $\rho(V)=1$, then there are just the following possibilities:

- $\quad H^{n}=1$ and $V=V_{6}$ is a weighted hypersurface in $\mathbb{P}\left(1^{n}, 2,3\right)$ of degree 6;

- $\quad H^{n}=2$ and $V=V_{4}$ is a weighted hypersurface in $\mathbb{P}\left(1^{n+1}, 2\right)$ of degree 4;

- $\quad H^{n}=3$ and $V=V_{3}$ is a cubic hypersurface in $\mathbb{P}^{n+1}$;

- $H^{n}=4$ and $V=V_{2 \cdot 2}$ is a complete intersection of two quadrics in $\mathbb{P}^{n+2}$;

- $H^{n}=5, n \in\{3,4,5,6\}$ and $V$ is described in Example 1.31.

If $\operatorname{dim}(V)=3$ and $\rho(V)=1$, then the values of the Hodge number $h^{1,2}(V)$ are given in the following table:

\begin{tabular}{cccccc}
\hline$H^{3}$ & 1 & 2 & 3 & 4 & 5 \\
\hline$h^{1,2}(V)$ & 21 & 10 & 5 & 2 & 0 \\
\hline
\end{tabular}

Let us prove cylindricity of any higher-dimensional smooth intersection of two quadrics.

Lemma 3.2 ([120]). Let $V$ be a smooth complete intersection of two quadric hypersurfaces in $\mathbb{P}^{n+2}$. Then $V$ is cylindrical.

Proof. Let $\ell$ be a line in $V$, let $D$ be an irreducible divisor in $X$ swept out by lines meeting $\ell$, let $\sigma: \widetilde{V} \rightarrow V$ be the blowup of the line $\ell$, let $E$ be its exceptional divisor, 
and let $\widetilde{D}$ be the proper transform on $\widetilde{V}$ of the divisor $D$. There exists the following commutative diagram:

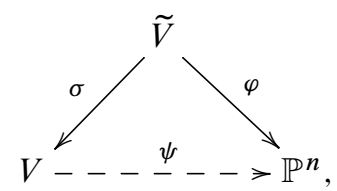

where $\varphi$ is a birational morphism that contracts $\widetilde{D}$, and $\psi$ is the projection from $\ell$. Thus, we have

$$
V \backslash D \cong \mathbb{P}^{n} \backslash \varphi(E) .
$$

But $\varphi(E)$ is a quadric that contains a one-parameter family of linear subspaces of dimension $n-2$. Hence, this quadric is singular, so that $\mathbb{P}^{n} \backslash \varphi(E)$ contains a cylinder.

Smooth Fano varieties of dimension $n \geqslant 3$ and index $n-2$ are known as Fano-Mukai varieties. If $V$ is a Fano-Mukai variety and $H \in \operatorname{Pic}(V)$ such that $-K_{V} \sim(n-2) H$, then the number

$$
\mathrm{g}(V)=\frac{1}{2} H^{n}+1
$$

is integral and is called the genus of the Fano-Mukai variety $V$. The possible values of the genus are given in the following table:

\begin{tabular}{cccccccc}
\hline $\mathrm{g}(V)$ & $2 \leqslant \mathrm{~g}(V) \leqslant 5$ & 6 & 7 & 8 & 9 & 10 & 12 \\
\hline $\operatorname{dim}(V)$ & any & $\leqslant 6$ & $\leqslant 10$ & $\leqslant 8$ & $\leqslant 6$ & $\leqslant 5$ & 3 \\
\hline
\end{tabular}

Moreover, the following result has been recently proved in [138].

Theorem 3.3. Let $V$ be a smooth Fano-Mukai variety such that $\rho(V)=1$ and $\mathrm{g}(V) \in$ $\{7,8,9,10\}$. Suppose that $\operatorname{dim}(V) \geqslant 5$. Then $V$ is cylindrical.

In Subsection 3.1, we will outline several known results about cylindrical smooth Fano threefolds. Then, in Subsection 3.2, we will present constructions of cylinders in some smooth Fano fourfolds. In particular, we will explain how to prove the following result:

Theorem 3.4. For every $g \in\{7,8,9,10\}$, there is a cylindrical Fano-Mukai fourfold of genus $g$.

Finally, in Subsection 3.3, we will present results about cylinders in Mori fibrations.

\subsection{Cylindrical Fano threefolds}

Let $X$ be a smooth Fano variety that has dimension three. Then $X$ belongs to one of 105 families, which have been explicitly described in [105-107, 110, 153-156]. Their automorphism groups have been studied in $[139,140,160,179,189]$. In particular, we have the following theorem. 
Theorem 3.5. Let $X$ be a smooth Fano threefold such that $\rho(X)=1$ and $\operatorname{Aut}(X)$ is infinite. Then $X$ and $\operatorname{Aut}(X)$ can be described as follows:

(1) $X=\mathbb{P}^{3}$ and $\operatorname{Aut}(X) \cong \mathrm{PGL}_{4}(\mathbb{k})$;

(2) $X$ is a smooth quadric in $\mathbb{P}^{4}$ and $\operatorname{Aut}(X) \cong \operatorname{PSO}_{5}(\mathbb{k})$;

(3) $X$ is the quintic del Pezzo threefold described in Example 1.31 and $\operatorname{Aut}(X) \cong$ $\mathrm{PGL}_{2}(\mathbb{k})$;

(4) $X$ is one of the following Fano threefolds in $\mathbb{P}^{13}$ of degree 22 and genus 12 :

(a) the Mukai-Umemura threefold $X_{22}^{\mathrm{mu}}$ with $\operatorname{Aut}\left(X_{22}^{\mathrm{mu}}\right) \cong \mathrm{PGL}_{2}(\mathbb{k})$;

(b) the unique special threefold $X_{22}^{\mathrm{a}}$ with $\operatorname{Aut}\left(X_{22}^{\mathrm{a}}\right) \cong \mathbb{G}_{\mathrm{a}} \rtimes \mu_{4}$;

(c) a threefold $X_{22}^{\mathrm{m}}$ in one-parameter family with $\operatorname{Aut}\left(X_{22}^{\mathrm{m}}\right) \cong \mathbb{G}_{\mathrm{m}} \rtimes \mu_{2}$.

Before we describe some cylindrical smooth Fano threefolds, observe that we have the following implications:

$X$ contains $\left(-K_{X}\right)$-polar cylinder $\Longrightarrow X$ is cylindrical $\Longrightarrow X$ is rational.

Moreover, the rationality problem for smooth Fano threefolds is almost completely solved (see [113]). In particular, for general member of every family, we know whether it is rational or irrational. It is expected that the same answer holds for every smooth member in each family.

If $\iota(X) \geqslant 3$, then either $X \cong \mathbb{P}^{3}$ or $X$ is a smooth quadric in $\mathbb{P}^{4}$, so that $X$ is cylindrical.

If $\iota(X)=2$, then $-K_{X} \sim 2 H$ for $H \in \operatorname{Pic}(X)$, and we have the following possibilities:

- $\quad H^{3}=1$ and $X=V_{1}$ is a sextic hypersurface in $\mathbb{P}(1,1,1,2,3)$;

- $H^{3}=2$ and $X=V_{2}$ is quartic hypersurface in $\mathbb{P}(1,1,1,1,2)$;

- $H^{3}=3$ and $X=V_{3}$ is a cubic hypersurface in $\mathbb{P}^{4}$;

- $H^{3}=4$ and $X=V_{4}$ is an intersection of two quadrics in $\mathbb{P}^{5}$;

- $H^{3}=5$ and $X=V_{5}$ is the quintic del Pezzo threefold described in Example 1.31;

- $H^{3}=6$ and $X$ is a divisor in $\mathbb{P}^{2} \times \mathbb{P}^{2}$ of degree $(1,1)$;

- $H^{3}=6$ and $X=\mathbb{P}^{1} \times \mathbb{P}^{1} \times \mathbb{P}^{1}$;

- $H^{3}=7$ and $X=V_{7}$ is a blowup of $\mathbb{P}^{3}$ at a point.

In this case, if $H^{3} \leqslant 3$, then $X$ is irrational (see [6,42,48,87,88,210]), so that it is not cylindrical. On the other hand, if $H^{3} \geqslant 4$, then $X$ contains a $\left(-K_{X}\right)$-polar cylinder. Indeed, if $H^{3}=4$, this follows from Lemma 3.2. If $H^{3} \geqslant 6$, this is obvious. Finally, if $H^{3}=5$, this follows from the following theorem.

Theorem 3.6. Let $V_{5}$ be the quintic del Pezzo threefold in $\mathbb{P}^{6}$ that is described in Example 1.31. Then $V_{5}$ contains a hyperplane section $H$ such that $V_{5} \backslash H \cong \mathbb{A}^{3}$. 
Proof. Let us give two constructions of the required hyperplane section. First, let $L$ be a line in $X$. Let $\alpha: \widetilde{V}_{5} \rightarrow V_{5}$ be the blowup of the line $L$. Then we have the following commutative diagram:

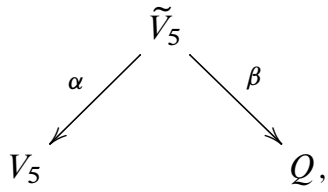

where $Q$ is a smooth quadric in $\mathbb{P}^{4}$, and $\beta$ is the blowup of a twisted cubic curve $C$ contained in $Q$. Let $H_{C}$ be the unique hyperplane section of $Q$ that contains $C$, and let $H_{L}$ be the unique hyperplane section of $V_{5}$ that is singular along $L$. Then $H_{L}$ is the proper transform of the $\beta$-exceptional surface, and $H_{C}$ is the proper transform of the $\alpha$-exceptional surface. Note that $H_{L}$ is swept out by the lines that intersects the line $L$. Moreover, it follows from $[113,140]$ that

$$
\mathcal{N}_{L / V_{5}} \cong \begin{cases}\mathcal{O}_{L} \oplus \mathcal{O}_{L} & L \text { is a line of type }(0,0), \\ \mathcal{O}_{L}(1) \oplus \mathcal{O}_{L}(-1) & L \text { is a line of type }(1,-1)\end{cases}
$$

The lines in $V_{5}$ are parametrized by $\mathbb{P}^{2}$, and the lines of the type $(1,-1)$ are parametrized by a smooth conic in this plane (see [80,107,140]). Furthermore, the surface $H_{C}$ is smooth if and only if $L$ is a line of type $(1,-1)$. Thus, if we choose $L$ to be a line of type $(1,-1)$ and put $H=H_{L}$, then $V_{5} \backslash H \cong Q \backslash H_{C} \cong \mathbb{A}^{3}$, as required.

To present the second construction, let $P$ be a point in $V_{5}$. Recall that $\operatorname{Aut}\left(V_{5}\right) \cong$ $\mathrm{PGL}_{2}(\mathbb{k})$. Moreover, it follows from [44,80,107, 140,160] that $\operatorname{Aut}\left(V_{5}\right)$ has exactly three orbits on $V_{5}$ :

(1) a closed one-dimensional orbit $\ell$, which is a twisted rational sextic curve in $\mathbb{P}^{6}$;

(2) a two-dimensional orbit $\stackrel{\circ}{\AA}$ whose closure is a surface $S \sim-K_{V}$ which is singular along $e$;

(3) an open orbit $V_{5} \backslash S$.

Furthermore, let $k_{P}$ be the number of lines in $V_{5}$ passing through $P$. Then

$$
k_{P}= \begin{cases}1 & \text { if } P \in \mathcal{C} \\ 2 & \text { if } S \backslash \mathcal{C} \\ 3 & \text { if } V_{5} \backslash \delta\end{cases}
$$

Observe also that $S$ is swept out by the lines of type $(1,-1)$.

Let $\sigma: \widehat{V}_{5} \rightarrow V_{5}$ be the blowup of the point $P$. Then it follows from [81] that there exists the following Sarkisov link:

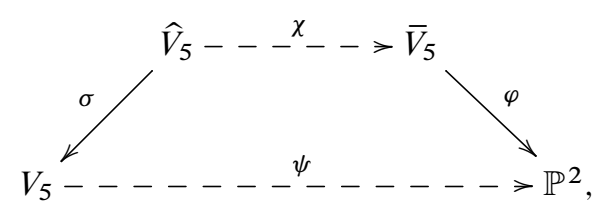


where $\chi$ is a composition of flops of the proper transforms of lines in $V_{5}$ that pass through $P$, the morphism $\varphi$ is a $\mathbb{P}^{1}$-bundle, and $\psi$ is given by the linear system of hyperplane sections that are singular at the point $P$. Now we suppose that $P \in \mathcal{C}$.

Let $E$ be the $\sigma$-exceptional surface, and let $\bar{E}$ be its proper transform on the threefold $\bar{V}_{5}$. Then $\bar{E}$ is a del Pezzo surface of degree 6 with at most Du Val singularities, and its singular locus consists of one singular point of type $\mathrm{A}_{2}$. Moreover, the $\mathbb{P}^{1}$-bundle $\varphi: \bar{V}_{5} \rightarrow \mathbb{P}^{2}$ induces a birational map $\bar{E} \rightarrow \mathbb{P}^{2}$ that contracts a single curve $\Gamma \subset \bar{E}$ to a point in $\mathbb{P}^{2}$.

Let $\mathcal{L}$ be a line in $\mathbb{P}^{2}$ that passes through the point $\varphi(\Gamma)$, let $\bar{H}$ be its preimage in $\bar{V}_{5}$ via $\varphi$, let $\hat{H}$ be its proper transform on $\widehat{V}_{5}$, and let $H=\sigma(\hat{H})$. Then

$$
V_{5} \backslash H \cong \bar{V}_{5} \backslash(\bar{E} \cup \bar{H})
$$

and $H$ is a hyperplane section of the threefold $V_{5}$ that is singular at $P$. Furthermore, one can show that the surface $H$ is smooth away from $P$, and $H$ has Du Val singularity of type $\mathrm{A}_{4}$ at this point. Then the $\mathbb{P}^{1}$-bundle $\varphi$ induces a morphism $\bar{V}_{5} \backslash(\bar{E} \cup \bar{H}) \rightarrow$ $\mathbb{P}^{2} \backslash \mathcal{L}$ that is an $\mathbb{A}^{1}$-bundle over $\mathbb{A}^{2}$. This implies that $V_{5} \backslash H \cong \bar{V}_{5} \backslash(\bar{E} \cup \bar{H}) \cong \mathbb{A}^{3}$, as required.

Now, we assume that $\iota(X)=1$. This leaves us 95 families of smooth Fano threefolds $[113,153]$. If $\rho(X)=1, \iota(X)=1$ and $\mathrm{g}(X) \leqslant 6$, then we have the following possibilities:

(1) $\mathrm{g}(X)=2$ and $X$ is a sextic hypersurface in $\mathbb{P}\left(1^{4}, 3\right)$;

(2) $\mathrm{g}(X)=3$ and $X$ is an intersection of a quadric and a quartic in $\mathbb{P}\left(1^{5}, 2\right)$;

(3) $\mathrm{g}(X)=4$ and $X$ is a complete intersection of a quadric and a cubic in $\mathbb{P}^{5}$;

(4) $\mathrm{g}(X)=5$ and $X$ is a complete intersection of three quadrics in $\mathbb{P}^{6}$;

(5) $\mathrm{g}(X)=6$ and $X$ is a section of the cone in $\mathbb{P}^{8}$ over the smooth quintic del Pezzo fourfold described in Example 1.31 by a quadric and a hyperplane.

All of these deformation families are irreducible. General members of the family (2) are smooth quartic hypersurfaces in $\mathbb{P}^{4}$, and special members are double covers of the quadric threefold branched over octic surfaces. Similarly, general members of the family (5) are sections of the smooth quintic del Pezzo fourfold in $\mathbb{P}^{7}$ by quadrics, and special members are double covers of the smooth quintic del Pezzo threefold branched over anticanonical surfaces.

In the first two cases, the Fano threefold $X$ is known to be irrational even if we allow mild isolated singularities [35, 104, 108, 109, 141, 148, 190, 203]. In the case (4), the threefold $X$ is also irrational [16]. General threefolds of the families (3) and (5) are irrational $[16,100,112,114,191]$, and every smooth member is also expected to be irrational. Therefore, in all these cases, the threefold $X$ is either non-cylindrical or it is expected to be irrational and, thus, non-cylindrical.

Remark 3.7. Let $V_{5}$ be the smooth quintic del Pezzo threefold, see Example 1.31, and let $\pi: X \rightarrow V_{5}$ be a double cover branched over a surface $S \in\left|-K_{V_{5}}\right|$. If $S$ has an isolated 
ordinary double point, then $X$ is rationally connected [215], it is $\mathbb{Q}$-factorial [50], and it follows from [183] that there exists the following Sarkisov link:

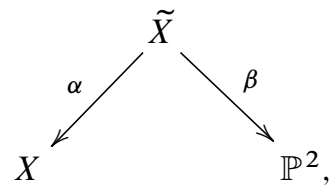

where $\alpha$ is the blow up of the singular point of $X$, and $\beta$ is a standard conic bundle, whose discriminant curve has degree 6 . Hence, in this case, the threefold $X$ is irrational by [202, Theorem 10.2]. Now, using [128, Theorem IV.1.8.3], we conclude that $X$ is also irrational if $S$ is a very general surface in the linear system $\left|-K_{V_{5}}\right|$.

If $\rho(X)=1, \iota(X)=1$ and $\mathrm{g}(X) \geqslant 7$, then $\mathrm{g}(X) \in\{7,8,9,10,12\}$. Moreover, if $\mathrm{g}(X)=8$, then the threefold $X$ is birational to a smooth cubic hypersurface in $\mathbb{P}^{4}$ (see, for example, [108, 113, 204]), so that it is irrational [48]. On the other hand, we know that $X$ is rational if

$$
\mathrm{g}(X) \in\{7,9,10,12\} .
$$

In these cases, the divisor $-K_{X}$ is very ample, and $\left|-K_{X}\right|$ gives an embedding $X \hookrightarrow$ $\mathbb{P}^{\mathrm{g}(X)+1}$. Moreover, all the known constructions of cylinders in $X$ use the double projection from a line in $X$ (see [110]). Recall from [113,122,178] that $X$ can contain two types of lines depending on their normal bundles. Namely, for a line $\ell \subset X$, we have the following two possibilities:

$$
\mathcal{N}_{\ell / X} \cong \begin{cases}\mathcal{O}_{\ell} \oplus \mathcal{O}_{\ell}(-1) & \ell \text { is of type }(0,-1), \\ \mathcal{O}_{\ell}(1) \oplus \mathcal{O}_{\ell}(-2) & \ell \text { is of type }(1,-2) .\end{cases}
$$

If $X$ is a sufficiently general member of one of these three families of smooth Fano threefolds, then $X$ does not contain lines of type $(1,-2)$. Moreover, one can show that the threefolds containing lines of type $(1,-2)$ form a codimension one subset in the corresponding moduli spaces. On the other hand, we have the following result:

Theorem 3.8 ([122, Theorem 0.1]). Suppose that $\rho(X)=1, \iota(X)=1$, and $\mathrm{g}(X)=9$ or $\mathrm{g}(X)=10$. If $X$ contains a line of type $(1,-2)$, then $X$ is cylindrical.

Proof. Let $\ell$ be a line in the Fano threefold $X$, and let $\sigma: \tilde{X} \rightarrow X$ be the blowup of the line $\ell$. Then it follows from $[107,110,113,180]$ that there is the Sarkisov link:

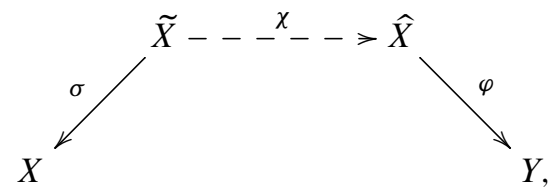

where $Y$ is a smooth Fano threefold described below, the morphism $\varphi$ is the blowup of a smooth rational curve $\Gamma$, and $\chi$ is a composition of flops of the proper transforms of the lines that meet $\ell$. Moreover, we have the following options: 
- if $\mathrm{g}(X)=9$, then $Y=\mathbb{P}^{3}$, and $\Gamma$ is a curve of degree 7 and genus 3;

- if $\mathrm{g}(X)=10$, then $Y$ is a smooth quadric in $\mathbb{P}^{4}$, and $\Gamma$ is a curve of degree 7 and genus 2 .

Let $E$ be the $\sigma$-exceptional surface, let $\widehat{E}$ be its proper transform on $\widehat{X}$, and let $\mathcal{S}=$ $\varphi(\widehat{E})$. Then $\mathcal{S}$ is a (maybe singular or non-normal) del Pezzo surface of degree $\mathrm{g}(X)-3$ that contains $\Gamma$. Similarly, let $S$ be the proper transform of the $\varphi$-exceptional surface on the Fano threefold $X$. Then $S$ is a hyperplane section of $X$ such that $\operatorname{mult}_{\ell}(S)=3$. Using this, we conclude that

$$
X \backslash S \cong Y \backslash \mathcal{S} \text {. }
$$

Moreover, if $\ell$ is a line of type $(1,-2)$, then the surface $\mathcal{S}$ is not normal. This implies that the complement $Y \backslash \mathcal{S}$ contains a cylinder, so that $X$ is cylindrical.

In fact, we believe that the following is true:

Conjecture 3.9. Let $X$ be a very general smooth Fano threefold such that $\rho(X)=1$, $\iota(X)=1$, and $\mathrm{g}(X)=9$ or $\mathrm{g}(X)=10$. Then $X$ is not cylindrical.

Using a similar Sarkisov link as in the proof of Theorem 3.8, we obtain the following: Theorem 3.10 ([120]). Suppose that $\rho(X)=1, \iota(X)=1$ and $g(X)=12$. Then $X$ is cylindrical.

Proof. Let $\ell$ be a line in $X$. Then there exists a unique surface $S \in\left|-K_{X}\right|$ such that mult $_{\ell}(S)=3$. Moreover, it follows from $[107,110,113,180]$ that there exists the following Sarkisov link:

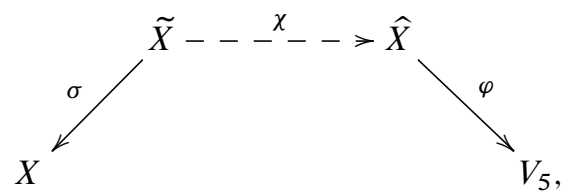

where $\sigma$ is the blowup of the line $\ell$, the variety $V_{5}$ is a smooth quintic del Pezzo threefold in $\mathbb{P}^{6}$, the morphism $\varphi$ is the blowup of a rational quintic curve $\Gamma$, and $\chi$ is a composition of flops.

Let $E$ be the $\sigma$-exceptional surface, let $\widehat{E}$ be its proper transform on $\widehat{X}$, and let $\mathcal{S}=$ $\varphi(\widehat{E})$. Then $\mathcal{S}$ is a hyperplane section of the threefold $V_{5}$ that contains the curve $\Gamma$, and $S$ is the proper transform of the $\varphi$-exceptional surface. Moreover, we have

$$
X \backslash S \cong V_{5} \backslash \mathcal{S} .
$$

Let us show that $V_{5} \backslash \mathcal{S}$ contains a cylinder. In fact, this follows from the proof of Theorem 3.6. We will use the notation and assumptions introduced in this proof.

Let $L$ be a line in $V_{5}$ that is contained in $\mathcal{S}$ (it does exists). If $\mathcal{S} \neq H_{L}$, let $S$ be the proper transform on $Q$ of the surface $\mathcal{S}$. Otherwise, we let $\mathrm{S}=H_{C}$. Then the surface $\mathrm{S}$ is a hyperplane section of the quadric $Q$. Thus, we see that

$$
V_{5} \backslash\left(\mathcal{S} \cup H_{L}\right) \cong Q \backslash\left(\mathrm{S} \cup H_{C}\right) .
$$


Now taking the linear projection $Q \rightarrow \mathbb{P}^{3}$ from a sufficiently general point in $\mathrm{S} \cap H_{C}$, one can easily show that the complement $Q \backslash\left(\mathrm{S} \cup H_{C}\right)$ contains a cylinder, so that $X$ is cylindrical.

Remark 3.12 ([180]). In the notation and assumptions of the proof of Theorem 3.10, let $\ell$ be a line of type $(-1,2)$. Then $\mathcal{S}$ is a non-normal surface whose singular locus is a line in $V_{5}$. Letting $L$ to be this line gives $\mathcal{S}=H_{L}$, so that

$$
X \backslash S \cong V_{5} \backslash \mathcal{S} \cong Q \backslash H_{C}
$$

Thus, if we also have $\mathcal{N}_{L / V_{5}} \cong \mathcal{O}_{L}(1) \oplus \mathcal{O}_{L}(-1)$, then $H_{C}$ is singular (see the proof of Theorem 3.6), so that $X \backslash S \cong \mathbb{A}^{3}$. We can always find such $\ell$ and $L$ if $\operatorname{Aut}(X)$ is infinite (see Theorem 3.5).

We do not know examples of cylindrical smooth Fano threefolds of Picard rank 1 and genus 7. In fact, we believe that any such threefold is not cylindrical.

Conjecture 3.13. Let $X$ be a smooth Fano threefold such that $\rho(X)=1, \iota(X)=1$, and $\mathrm{g}(X)=7$. Then $X$ is not cylindrical.

Before we close this section, let us mention that most of smooth Fano threefolds with $\rho(X) \geqslant 2$ are rational $[112,113,184]$, and many of them are known to be cylindrical. However, we do not know the existence of anticanonical polar cylinders in majority of cylindrical smooth Fano threefolds. Let us list few examples.

Example 3.14. Let $Y$ be a smooth Fano threefold such that $Y$ is a del Pezzo threefold or $Y=\mathbb{P}^{3}$. Take $H \in \operatorname{Pic}(Y)$ on $Y$ such that $-K_{Y} \sim 2 H$. Choose a smooth curve $\mathcal{C} \subset Y$ that is a complete intersection of two surfaces from $|H|$. Suppose that $X$ is a blowup of the threefold $Y$ along $\mathcal{C}$. Then $X$ is a smooth Fano threefold. Moreover, if $H^{3} \geqslant 4$, then $X$ is cylindrical.

Example 3.15. Suppose that $X$ is a blowup of $\mathbb{P}^{3}$ along a smooth curve that is a complete intersection of two cubic surfaces. Then $X$ is a cylindrical smooth Fano threefold.

Example 3.16. Suppose that $X$ is a blowup of $\mathbb{P}^{3}$ along a smooth curve of degree 6 and genus 3, which is an intersection of cubic hypersurfaces. Then $X$ is a cylindrical smooth Fano threefold.

Example 3.17. Let $Q$ be a smooth quadric threefold in $\mathbb{P}^{4}$, and let $H$ be its hyperplane section. Suppose that $X$ is a blowup of $Q$ along a smooth curve that is a complete intersection of two surfaces from $|2 H|$. Then $X$ is a cylindrical smooth Fano threefold.

Each smooth Fano threefold described in Examples 3.14, 3.15, 3.16 and 3.17 is cylindrical, but we do not know whether any of these threefolds contains anticanonical polar cylinders or not. 


\subsection{Cylindrical Fano fourfolds}

Now, let $X$ be a smooth Fano fourfold such that $\rho(X)=1$. By Corollary 1.6 we have the following implications:

$$
X \text { is cylindrical } \Longrightarrow X \text { is rational. }
$$

If $\iota(X)=5$ or $\iota(X)=4$, then $X=\mathbb{P}^{4}$ or $X$ is a smooth quadric fourfold, so that $X$ is cylindrical. Similarly, if $\iota(X)=3$, then it follows from Remark 3.1 that $X$ is one of the following fourfolds:

(1) a smooth sextic hypersurface in $\mathbb{P}(1,1,1,1,2,3)$;

(2) a smooth quartic hypersurface in $\mathbb{P}(1,1,1,1,1,2)$;

(3) a smooth cubic fourfold in $\mathbb{P}^{5}$;

(4) a smooth complete intersection of two quadrics in $\mathbb{P}^{6}$;

(5) the quintic del Pezzo fourfold described in Example 1.31.

In the first two cases, we expect that $X$ is always irrational. In fact, we know that a very general quartic hypersurface in $\mathbb{P}(1,1,1,1,1,2)$ is irrational [97], so that it is definitely not cylindrical. Similarly, general cubic fourfold in $\mathbb{P}^{5}$ is expected to be irrational. But there are rational smooth cubic fourfolds (see [95,96,196, 207]), so that it is very natural to ask the following question:

Question 3.18. Are there smooth rational cylindrical cubic fourfolds?

Remark 3.19. Every smooth cubic fourfold in $\mathbb{P}^{5}$ containing two skew planes is rational (see [96]). In particular, the Fermat cubic fourfold is rational. If it is cylindrical, then the affine cone over it admits an effective action of the group $\mathbb{G}_{\mathrm{a}}$ by Theorem 1.15 , which contradicts Conjecture 1.22.

By Lemma 3.2, we know that a smooth complete intersection of two quadrics in $\mathbb{P}^{6}$ is cylindrical. Let us prove that the quintic del Pezzo fourfold described in Example 1.31 is cylindrical as well. To do this, let us present a detailed description of this fourfold given in [182].

Let $V_{5}$ be the quintic del Pezzo fourfold in $\mathbb{P}^{7}$. By [175, Theorem 6.6], we have the following exact sequence of groups:

$$
1 \longrightarrow\left(\mathbb{G}_{\mathrm{a}}\right)^{4} \rtimes \mathbb{G}_{\mathrm{m}} \longrightarrow \operatorname{Aut}\left(V_{5}\right) \longrightarrow \mathrm{PGL}_{2}(\mathbb{C}) \longrightarrow 1,
$$

so that the group $\operatorname{Aut}\left(V_{5}\right)$ is not reductive. In particular, the fourfold $V_{5}$ is not K-polystable [2]. The planes on $V_{5}$ belong to one of the following two classes:

(i) a unique plane $\Xi$ which is a Schubert variety of type $\sigma_{2,2}$;

(ii) a one-parameter family of planes $\Pi_{t}$ that are Schubert varieties of type $\sigma_{3,1}$.

We say that $\Xi$ is the plane of type $\sigma_{2,2}$, and $\Pi_{t}$ are planes of type $\sigma_{3,1}$. They are distinguished by the types of the normal bundles: $c_{2}\left(\mathcal{N}_{\Xi / X}\right)=2$ and $c_{2}\left(\mathcal{N}_{\Pi_{t} / X}\right)=1$. Moreover, 
there is a hyperplane section $\mathscr{H}$ of the fourfold $V_{5}$ that contains all planes in $V_{5}$. Furthermore, one has $\operatorname{Sing}(\mathscr{H})=\Xi$, the threefold $\mathscr{H}$ is the union of all the $\sigma_{3,1}$-planes in $V_{5}$, and $\Xi$ contains a special conic $\mathcal{C}$ such that

- the intersection $\Pi_{t} \cap \Xi$ is a tangent line to the conic $\ell$;

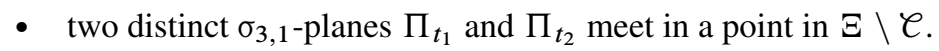

The automorphism group $\operatorname{Aut}\left(V_{5}\right)$ has the following orbits in $V_{5}$ :

(1) the open orbit $X \backslash \mathscr{H}$;

(2) the three-dimensional orbit $\mathscr{H} \backslash \Xi$;

(3) the two-dimensional orbit $\Xi \backslash \ell$;

(4) the one-dimensional closed orbit $e$.

The Hilbert scheme of lines on the del Pezzo fourfold $V_{5}$ is smooth, irreducible, and fourdimensional. Moreover, if $\ell$ is a line in $V_{5}$, then $\ell$ belongs to one of the following five classes:

(a) $\ell \not \subset \mathscr{H}, \ell \cap \Xi=\varnothing$, and $l \cap \mathscr{H}$ is a point;

(b) $\ell \subset \mathscr{H}, l \cap \Xi$ is a point, and $\ell \cap \ell=\varnothing$;

(c) $\ell \subset \mathscr{H}$, and $l \cap \Xi=l \cap \mathcal{Y}$ is a point;

(d) $\ell \subset \Xi$, and the intersection $\ell \cap \mathcal{C}$ consists of two points;

(e) $\ell \subset \Xi$ and $\ell$ is tangent to $\ell$.

The group $\operatorname{Aut}\left(V_{5}\right)$ acts transitively on the lines in each of these classes. For a line $\ell \subset V_{5}$, the lines meeting $\ell$ sweep out a hyperplane section $H_{\ell}$ of the fourfold $V_{5}$ that is singular along the line $\ell$. Vice versa, if $H$ is a hyperplane section of the quintic del Pezzo fourfold $V_{5}$ that has non-isolated singularities, then $H=H_{\ell}$ for some line $\ell \subset V_{5}$.

Theorem 3.20 ([182]). Let $\ell$ be a line in $V_{5}$ that is not a line of type (b). Then $V_{5} \backslash H_{\ell} \cong \mathbb{A}^{4}$.

Proof. If $\ell$ is a line of type (d) or (e), then $H_{\ell}=\mathscr{H}$. On the other hand, there exists the following $\operatorname{Aut}\left(V_{5}\right)$-equivariant Sarkisov link:

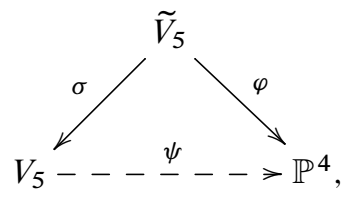

where $\sigma$ is the blowup of the plane $\Xi, \varphi$ is the blowup of a twisted cubic curve $C$, and $\psi$ is the linear projection from $\Xi$. Then the $\varphi$-exceptional divisor is the proper transform of the threefold $\mathscr{H}$. Moreover, if $E$ is the $\sigma$-exceptional divisor, then $\varphi(E)$ is the hyperplane in $\mathbb{P}^{4}$ that contains $C$. Thus, if $\ell$ is a line of type (d) or (e), then

$$
V_{5} \backslash H_{\ell}=V_{5} \backslash \mathscr{H} \cong \mathbb{P}^{4} \backslash \varphi(E) \cong \mathbb{A}^{5} .
$$


Let $\pi: \widehat{V}_{5}$ be the blowup of the line $\ell$. Then there exists the following Sarkisov link:

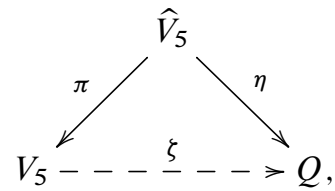

where $Q$ is an irreducible quadric in $\mathbb{P}^{5}$, the map $\zeta$ is the projection from $\ell$, and $\eta$ is a birational morphism that contracts the proper transform of the hyperplane section $H_{\ell}$ to a surface of degree 3. Let $\widehat{H}_{\ell}$ be the proper transform on $\widehat{V}_{5}$ of the threefold $H_{\ell}$, and let $F$ be the $\pi$-exceptional divisor. Then $V_{5} \backslash H_{\ell} \cong Q \backslash \eta(F)$, and $\eta(F)$ is a singular hyperplane section of the quadric $Q$.

If $\ell$ is a line of type (a), then all fibers of $\eta$ are one-dimensional, so that $Q$ is smooth (see [3]). Thus, in this case, we have $V_{5} \backslash H_{\ell} \cong Q \backslash \eta(F) \cong \mathbb{A}^{4}$.

To complete the proof, we may assume that $\ell$ is of type (c). Then $\ell$ is contained in a plane in $V_{5}$, so that $\eta$ has a two-dimensional fiber. Hence, in this case, the quadric $Q$ can be singular (cf. [4]). Analyzing the situation more carefully, we see that

$$
V_{5} \backslash H_{\ell} \cong Q \backslash \eta(F) \cong \mathbb{A}^{4}
$$

Corollary 3.22. The quintic del Pezzo fourfold is cylindrical.

In the remaining part of this subsection, we present known constructions of cylinders in some smooth Fano-Mukai fourfolds. Basically, our main goal is to explain how to prove Theorem 3.4. Thus, we suppose that $X$ is a smooth Fano-Mukai fourfold, $\rho(X)=1$ and $\mathrm{g}(X) \in\{7,8,9,10\}$.

Let $H$ be an ample Cartier divisor on $X$ such that

$$
-K_{X} \sim 2 H
$$

Then $H^{4}=2 \mathrm{~g}(X)-2 \in\{12,14,16,18\}$. Moreover, the divisor $H$ is very ample, and the linear system $|H|$ gives an embedding $X \hookrightarrow \mathbb{P}^{\mathrm{g}(X)+2}$. Let us deal with four cases separately.

If $\mathrm{g}(X)=10$, then $X=X_{18}$ is a hyperplane section of the homogeneous fivefold $G_{2} / P \subset \mathbb{P}^{13}$, where $G_{2}$ is the simple algebraic group of exceptional type $G_{2}$, and $P$ is its parabolic subgroup that corresponds to a short root (see $[158,159])$. The family $\mathfrak{X}$ of all such fourfolds is one-dimensional. Moreover, if $X=X_{18}$ is a general member of $\mathfrak{X}$, then $\operatorname{Aut}(X) \cong \mathbb{G}_{\mathrm{m}}^{2} \rtimes \mu_{2}$. Besides, there are three distinguished fourfolds in this family:

(0) $X_{18}^{\mathrm{r}}$ such that $\operatorname{Aut}\left(X_{18}^{\mathrm{r}}\right) \cong \mathbb{G}_{\mathrm{m}}^{2} \rtimes \mu_{6}$;

(1) $X_{18}^{\mathrm{s}}$ such that $\operatorname{Aut}\left(X_{18}^{\mathrm{s}}\right) \cong \mathrm{GL}_{2}(\mathbb{C}) \rtimes \mu_{2}$;

(2) $X_{18}^{\mathrm{a}} \operatorname{such}$ that $\operatorname{Aut}\left(X_{18}^{\mathrm{a}}\right) \cong\left(\mathbb{G}_{\mathrm{a}} \times \mathbb{G}_{\mathrm{m}}\right) \rtimes \mu_{2}$.

See [188] for details, where the following result has been proved: 
Theorem 3.23 ([188]). Let $X$ be a smooth Fano-Mukai fourfold in $\mathbb{P}^{12}$ of genus 10 with $\rho(X)=1$. Then there exists an $\operatorname{Aut}^{0}(X)$-invariant hyperplane section $H$ of $X$ such that the complement $X \backslash H$ is $\operatorname{Aut}^{0}(X)$-equivariantly isomorphic to $\mathbb{A}^{4}$.

This theorem implies, in particular, that any smooth Fano-Mukai fourfolds of genus 10 is cylindrical. See also Example 4.16 for another application of Theorem 3.23.

If $\mathrm{g}(X)=8$, then $X=X_{14}$ is a section of the Grassmannian $\operatorname{Gr}(2,6) \subset \mathbb{P}^{14}$ by a linear subspace of dimension 10 (see $[158,159])$. Some of these fourfolds are cylindrical.

Example 3.24 ([186]). Suppose that $\mathrm{g}(X)=8$ and $X$ contains a plane $\Pi$ which is a Schubert variety of type $\sigma_{4,2}$, and $X$ does not contain planes meeting $\Pi$ along a line. Such fourfolds do exist and form a subspace of codimension one in the moduli space of all Fano-Mukai fourfolds of genus 8. Then it follows from [181] that there exists the following Sarkisov link:

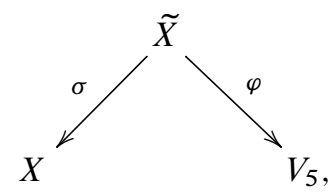

where $V_{5}$ is the del Pezzo quintic fourfold in $\mathbb{P}^{7}$ (see Theorem 3.20), $\sigma$ is the blowup of the plane $\Pi$, and $\varphi$ is the blowup of a smooth rational surface $S$ of degree 7 such that $K_{S}^{2}=3$. Then

$$
X \backslash H_{X} \cong V_{5} \backslash H_{V_{5}},
$$

where $H_{V_{5}}$ is the proper transform on $V_{5}$ of the $\sigma$-exceptional divisor, and $H_{X}$ is the proper transform on $X$ of the $\varphi$-exceptional divisor. On the other hand, the divisor $H_{V_{5}}$ is a hyperplane section of the fourfold $V_{5}$ that contains $S$, and $H_{X}$ is a hyperplane section of $X$ containing $\Pi$. Thus, the set $V_{5} \backslash H_{V_{5}}$ contains a cylinder by [186, Theorem 4.1], so that $X$ is cylindrical.

If $\mathrm{g}(X)=7$, then $X=X_{12}$ is a section of the orthogonal Grassmannian $\operatorname{OGr}(4,9) \subset \mathbb{P}^{15}$ by a linear subspace of dimension 9 (see $[158,159])$. In this case, we also have cylindrical fourfolds.

Example 3.25 ([186]). Suppose that $\mathrm{g}(X)=7$ and $X$ contains a plane $\Pi$. Such fourfolds do exist. Suppose that $X$ is a sufficiently general Fano-Mukai fourfold of genus 7 that contains the plane $\Pi$. Then by [181] there exists the Sarkisov link

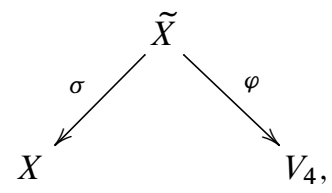

where $V_{4}$ is a smooth complete intersection of two quadrics in $\mathbb{P}^{6}, \sigma$ is the blowup of the plane $\Pi$, and $\varphi$ is the blowup of a smooth del Pezzo surface $S$ such that $K_{S}^{2}=5$. Arguing as in Example 3.24, we conclude that $X$ is cylindrical. 
If $\mathrm{g}(X)=9$, then $X=X_{16}$ is a section of the Lagrangian Grassmannian $\operatorname{LGr}(3,6) \subset \mathbb{P}^{13}$ by a linear subspace of dimension 11 (see $[158,159]$ ). There are cylindrical fourfolds in this family.

Example 3.26 ([187]). Suppose that $\mathrm{g}(X)=9$. Then $X_{16}$ contains an irreducible twodimensional quadric surface $S$. Suppose, for simplicity, that $X_{16}$ is a general Fano-Mukai fourfold of genus 9 that contains $S$. Then there exists the following Sarkisov link:

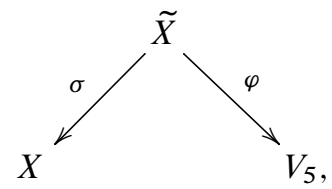

where $V_{5}$ is the del Pezzo quintic fourfold, $\sigma$ is the blowup of the surface $S$, and $\varphi$ is the blowup along a smooth del Pezzo surface of degree 6. Arguing as in Example 3.24, we see that $X$ is cylindrical.

The interested reader can consult also the recent preprint [94] for further examples of cylindrical Fano fourfolds.

\subsection{Cylinders in Mori fibrations}

This subsection is inspired by the following question.

Question 3.27. Given a family of cylindrical varieties, when its total space is cylindrical?

For example, irrational three-dimensional conic bundles are not cylindrical, though their general fibers are. In general, this question is very subtle and has birational nature, so that it is natural to consider it for Mori fibred spaces first.

Let $V$ be a projective variety with terminal $\mathbb{Q}$-factorial singularities, let $\pi: V \rightarrow B$ be a dominant projective non-birational morphism such that $-K_{V}$ is $\pi$-ample, $\pi_{*} \mathcal{O}_{V}=\mathcal{O}_{B}$ and $\rho(V)=\rho(B)+1$. Let $X_{\eta}$ be the fiber of the morphism $\pi$ over the (scheme-theoretic) generic point $\eta$ of the base $B$. Then $X_{\eta}$ is a Fano variety that has at most terminal singularities, which is defined over $\mathbb{K}=\mathbb{k}(B)$, i.e. the field of rational functions on $B$. Over the (algebraically non-closed) field $\mathbb{K}$, the divisor class group of the Fano variety $X_{\eta}$ is of rank 1 , because we assume that $\rho(V)=\rho(B)+1$.

Definition 3.28 ([59]). If the variety $V$ contains a (Zariski open) cylinder $U=\mathbb{A}^{1} \times Z$, we say that the cylinder $U$ is vertical (with respect to $\pi$ ) if there is a morphism $h: Z \rightarrow B$ such that the restriction $\left.\pi\right|_{U}: U \rightarrow B$ is a composition $h \circ \operatorname{pr}_{Z}$, where $\operatorname{pr}_{Z}: U \rightarrow Z$ is the natural projection. In this case, we have commutative diagram:

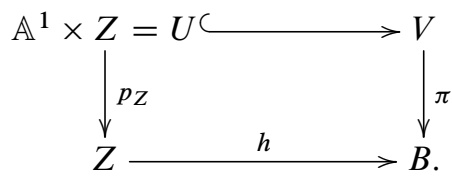

A cylinder in $V$ which is not vertical is called twisted. 
If $V$ contains a vertical cylinder $U=\mathbb{A}^{1} \times Z$, then the Fano variety $X_{\eta}$ contains a cylinder

$$
U_{\eta}=\mathbb{A}^{1} \times Z_{\eta},
$$

where $U_{\eta}$ and $Z_{\eta}$ are generic (scheme) fibers of the morphisms $h \circ \operatorname{pr}_{Z}$ and $h$ in (3.29), respectively. Vice versa, if the Fano variety $X_{\eta}$ contains a cylinder defined over the field $\mathbb{K}$, then $V$ does contain a vertical cylinder by [59, Lemma 3]. This gives a motivation to study cylinders in Fano varieties defined over arbitrary fields (cf. [18,99, 137, 138]) The first step in this direction is

Theorem 3.30 ([59]). Let $S$ be a geometrically irreducible smooth del Pezzo surface defined over a field $\mathbb{F}$ of characteristic 0. Suppose that $\rho(S)=1$. Then the following conditions are equivalent:

(i) the surface $S$ contains a cylinder defined over $\mathbb{F}$;

(ii) the surface $S$ is rational over $\mathbb{F}$;

(iii) $K_{S}^{2} \geqslant 5$ and $S$ has an $\mathbb{F}$-point.

Proof. It is commonly known that the conditions (ii) and (iii) are equivalent (see, for example, [111]). Moreover, the implication (iii) $\Rightarrow$ (i) can be shown using well-known Sarkisov links that start at $S$, which are described in [111]. For details, see the proof of [59, Proposition 12]. Thus, we just have to show that (i) implies (iii). This can also be shown using Sarkisov links, but we present another proof.

Suppose that $S$ contains a cylinder $U$ which is defined over $\mathbb{F}$. Then $U \cong \mathbb{A}^{1} \times Z$ for some affine curve $Z$ defined over $\mathbb{F}$. Let $\bar{Z}$ be the completion of the curve $Z$. Then $\bar{Z}$ is a geometrically irreducible curve. Moreover, we have the following commutative diagram

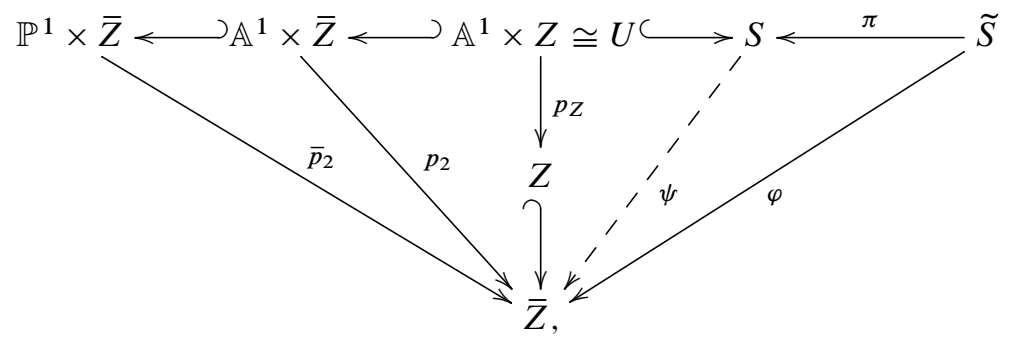

where $p_{Z}, p_{2}$ and $\bar{p}_{2}$ are the natural projections to the second factors, $\psi$ is the rational map induced by $p_{Z}, \pi$ is a birational morphism resolving the indeterminacy of $\psi$ and $\varphi$ is a morphism. By construction, a general fiber of $\varphi$ is isomorphic to $\mathbb{P}^{1}$.

Let $\Gamma$ be the section of $\bar{p}_{2}$ that is the complement of $\mathbb{A}^{1} \times \bar{Z}$ in $\mathbb{P}^{1} \times \bar{Z}$, and let $\widetilde{\Gamma}$ be the proper transform on $\widetilde{S}$ of the curve $\Gamma$. Then $\widetilde{\Gamma} \cong \Gamma \cong \bar{Z}$, the curve $\widetilde{\Gamma}$ is a section of $\varphi$, and the curve $\widetilde{\Gamma}$ is $\pi$-exceptional, because $\rho(S)=1$. Let $P=\pi(\widetilde{\Gamma})$. Then $P$ is an $\mathbb{F}$-point.

Now, we can proceed in two (slightly different) ways. First, as in the proof of [59, Theorem 1], we can let $\mathcal{M}$ to be the linear system on $S$ that gives the map $\psi$. Then, 
arguing as in Section 2.2, we conclude that $(S, \lambda \mathcal{M})$ is not log canonical at $P$ for a some $\lambda \in \mathbb{Q}_{>0}$ such that $\lambda \mathcal{M} \sim_{\mathbb{Q}}-K_{S}$. Such number exists, since $\rho(S)=1$. Let $M_{1}$ and $M_{2}$ be two general curves in $\mathcal{M}$. Then

$$
\frac{K_{S}^{2}}{\lambda^{2}}=M_{1} \cdot M_{2} \geqslant\left(M_{1} \cdot M_{2}\right)_{P}>\frac{4}{\lambda^{2}}
$$

by [49, Theorem 3.1]. This gives $K_{S}^{2} \geqslant 5$, so that (i) implies (iii).

Alternatively, we can use Corollary 2.9. Let $C_{1}, \ldots, C_{n}$ be the irreducible curves in $S$ that lie in the complement $S \backslash U$. Then we put $D=\lambda\left(C_{1}+\cdots+C_{n}\right)$ for $\lambda \in \mathbb{Q}_{>0}$ such that $D \sim \mathbb{Q}-K_{S}$. Therefore, we conclude that $S$ contains a $\left(-K_{S}\right)$-polar cylinder, so that $K_{S}^{2} \geqslant 4$ by Corollary 2.9. Thus, we may assume that $K_{S}^{2}=4$. Then our point $P$ is not contained in any $(-1)$-curve in $S \otimes_{\mathbb{F}} \overline{\mathbb{F}}$, where $\overline{\mathbb{F}}$ is an algebraic closure of the field $\mathbb{F}$. Indeed, otherwise the $\mathrm{Gal}(\overline{\mathbb{F}} / \mathbb{F})$-orbit of this curve would consist of at least four $(-1)$ curves that all pass through the point $P$, which is impossible. Let $\xi: \widehat{S} \rightarrow S$ be the blowup of the point $P$, and let $E$ be the exceptional curve of the blowup $\xi$. Then $\widetilde{S}$ is a smooth del Pezzo surface of degree $K_{\widetilde{S}}^{2}=3$ and

$$
\widetilde{D}+\left(\operatorname{mult}_{P}(D)-1\right) E \sim \mathbb{Q}-K_{\widehat{S}},
$$

where $\operatorname{mult}_{P}(D)>1$ by Remark 2.3 and Lemma A.3. Then $\widetilde{S}$ contains a $\left(-K_{\tilde{S}}\right)$-polar cylinder, which is impossible by Corollary 2.9. This again shows that (i) implies (iii).

Corollary 3.31 ([59, Theorem 1]). Suppose that $X_{\eta}$ is a del Pezzo surface. Then $V$ contains a vertical cylinder $\Longleftrightarrow K_{X_{\eta}}^{2} \geqslant 5$ and $\pi$ has a rational section.

Note that if $\mathbb{k}$ is uncountable and the general fiber of $\pi$ contains a cylinder, then it follows from [61,116] that the total space of the family $V \times_{B} B^{\prime} \rightarrow B$ contains a vertical cylinder for an appropriate finite base change $B^{\prime} \rightarrow B$. This basically means that $X_{\eta} \otimes_{\mathbb{K}} \mathbb{K}^{\prime}$ contains a cylinder defined over $\mathbb{K}^{\prime}$ for an appropriate finite extension of fields $\mathbb{K} \subset \mathbb{K}^{\prime}$.

Remark 3.32. If $X_{\eta}$ is a del Pezzo surface and $K_{X_{\eta}}^{2} \leqslant 4$, then $V$ can contain twisted cylinders. In fact, there are three-dimensional examples constructed in [58,59] such that $K_{X_{\eta}}^{2} \leqslant 3, B=\mathbb{P}^{1}$, and $V$ contains a Zariski open subset isomorphic to $\mathbb{A}^{3}$. See also [57, 90, 198, 199].

Now let us mention one relevant result about forms of the quintic del Pezzo threefold defined over a non-algebraically closed field (cf. [137, Theorem 3.3]).

Theorem 3.33 ([60]). Let $X$ be a smooth Fano threefold defined over a field $\mathbb{F}$ of characteristic 0. Suppose that $X \otimes_{\mathbb{F}} \overline{\mathbb{F}} \cong V_{5}$, where $V_{5}$ is the quintic del Pezzo threefold described in Example 1.31, where $\overline{\mathbb{F}}$ is the algebraic closure of the field $\mathbb{F}$. Then the following assertions hold:

- $\quad X$ contains a Zariski open subset $U \cong \mathbb{A}^{2} \times Z$ for some affine curve $Z$;

- $X$ contains a Zariski open subset isomorphic to $\mathbb{A}^{3}$ if and only if $X$ contains a smooth rational curve $\ell$ defined over $\mathbb{F}$ such that $-K_{V_{5}} \cdot \ell=2$ and $\mathcal{N}_{\ell / X} \cong \mathcal{O}_{\ell}(-1) \oplus \mathcal{O}_{\ell}(1)$. 
Let us conclude this section with the following generalization of Theorem 3.3.

Theorem 3.34 ([138]). Let $X$ be a smooth Fano threefold defined over a field $\mathbb{F}$ of characteristic 0. Suppose that $X \otimes_{\mathbb{F}} \overline{\mathbb{F}} \cong X_{2 g-2}$, where $X_{2 g-2}$ is a Fano-Mukai variety of genus $g$ with $\rho\left(X_{2 g-2}\right)=1$, where $\overline{\mathbb{F}}$ be the algebraic closure of $\mathbb{F}$. Suppose that the following conditions hold:

(1) $\operatorname{dim}(X) \geqslant 5$;

(2) $g \in\{7,8,9,10\}$;

(3) $X$ has an $\mathbb{F}$-point.

Then $X$ is cylindrical over $\mathbb{F}$.

\section{Beyond cylindricity}

\subsection{Flexible affine varieties}

Let $X$ be an affine variety. Given a $\mathbb{G}_{\mathrm{a}}$-action on $X$, it induces a representation of the group $\mathbb{G}_{\mathrm{a}}$ on the structure $\mathbb{k}$-algebra $\mathcal{O}(X)$ of the form

$$
(t, f) \longmapsto \exp (t \partial)(f)
$$

for $t \in \mathbb{G}_{\mathrm{a}}$ and $f \in \mathcal{O}(X)$, where the infinitesimal generator $\partial$ of the $\mathbb{G}_{\mathrm{a}}$-subgroup is a locally nilpotent derivation of $\mathcal{O}(X)$, which means that every element $f \in \mathcal{O}(X)$ is annihilated by $\partial^{(m)}$ for some sufficiently large $m$ that depends on the element $f$. Conversely, any locally nilpotent derivation of the $\mathbb{k}$-algebra $\mathcal{O}(X)$ generates a $\mathbb{G}_{\mathrm{a}}$-action on $X$ (see [65]).

Recall that the derivations of $\mathcal{O}(X)$ correspond to the regular vector fields on $X$. We say that a vector field on $X$ is locally nilpotent if the corresponding derivation is.

If an open variety $X$ admits a $\mathbb{G}_{\mathrm{a}}$-action, then the log-Kodaira dimension of $X$ is negative. However, the converse does not hold, in general. Indeed, there are smooth affine surfaces of negative log-Kodaira dimension which admit no effective $\mathbb{G}_{\mathrm{a}}$-action [91]. Let us stay on this in more detail.

As we mentioned already, any smooth affine surface $X$ of negative log-Kodaira dimension contains a cylinder [150, Ch. 2, Theorem 2.1.1]. Moreover, $X$ is affine-ruled, that is, there is a morphism $X \rightarrow C$ onto a smooth curve $C$ with general fiber $\mathbb{A}^{1}$. The base curve $C$ could be affine or projective. However, a smooth affine surface $X$ admits an effective $\mathbb{G}_{\mathrm{a}}$-action if and only if it admits an $\mathbb{A}^{1}$-ruling $X \rightarrow C$ over an affine curve $C$, or, which is equivalent, a principal cylinder, see Theorem 1.13. In [91] there are examples of smooth rational affine surfaces $\mathbb{A}^{1}$-ruled over $\mathbb{P}^{1}$ and with no $\mathbb{A}^{1}$-ruling over an affine curve. Hence, such a surface admits no effective $\mathbb{G}_{\mathrm{a}}$-action.

To construct such a surface $X$ we start with the quadric $\mathbb{P}^{1} \times \mathbb{P}^{1}$ endowed with the first projection to $\mathbb{P}^{1}$. We blow up three distinct points on the section $S=\mathbb{P}^{1} \times\{\infty\}$ and infinitesimally near points in such a way that each of the 3 resulting reducible fibers has a 
unique (-1)-component of multiplicity 2 and the union of the section $S$ and the remaining components of the reducible fibers forms a connected divisor $D$. The complement of $D$ in the resulting projective surface is a smooth affine surface $X$. It comes equipped with an $\mathbb{A}^{1}$-fibration $X \rightarrow \mathbb{P}^{1}$. Each fiber of this fibration is irreducible, and three of them are multiple of multiplicity 2. According to [92, Theorem 4.1], such a surface $X$ does not carry any $\mathbb{A}^{1}$-fibration over an affine curve. Hence, $X$ admits no $\mathbb{G}_{\mathrm{a}}$-action.

Definition 4.1. A point $P \in X$ is said to be flexible if locally nilpotent vector fields on $X$ span the tangent space $T_{P} X$. The variety $X$ is said to be flexible if every smooth point of $X$ is flexible. We also say that $X$ is generically flexible if every point in a non-empty Zariski open subset of $X$ is flexible.

Let $\operatorname{SAut}(X)$ be the subgroup of $\operatorname{Aut}(X)$ generated by all the $\mathbb{G}_{\mathrm{a}}$-subgroups. The flexibility of $X$ is ultimately related to the transitivity of the action of the group $\operatorname{SAut}(X)$. Indeed, we have the following criteria of flexibility.

Theorem 4.2 ([9]). Suppose that $\operatorname{dim}(X) \geqslant 2$. Then the following conditions are equivalent:

(1) the variety $X$ is flexible;

(2) the group $\operatorname{SAut}(X)$ acts transitively on the smooth locus of $X$;

(3) the group $\mathrm{SAut}(X)$ acts highly transitively on the smooth locus of $X$.

One says that a group acts highly transitively ${ }^{1}$ on an infinite set if it acts $m$-transitively for any natural number $m$.

Remark 4.3. A dimension count shows that an algebraic group cannot act highly transitively on an affine variety. Moreover, it cannot act even 3-transitively on an affine variety [22, 124].

Let us present examples of flexible affine varieties; see e.g. [11, 13, 14] for further examples.

Example 4.4. Let $X=\mathbb{A}^{n}$, where $n \geqslant 2$. Then the subgroup of translations in $\operatorname{SAut}\left(\mathbb{A}^{n}\right)$ acts transitively on the variety $X$, so that $X$ is flexible by Theorem 4.2 (cf. [117]).

Example 4.5. Let $X$ be the $n$th Calogero-Moser space defined as follows:

$$
\left\{(A, B) \in \operatorname{Mat}_{n}(\mathbb{k}) \times \operatorname{Mat}_{n}(\mathbb{k}) \mid \operatorname{rk}\left([A, B]+I_{n}\right)=1\right\} / / \operatorname{PGL}_{n}(\mathbb{k}),
$$

where $\mathrm{PGL}_{n}(\mathbb{k})$ acts via $g .(A, B)=\left(g A g^{-1}, g B g^{-1}\right)$. Then $X$ is a smooth rational irreducible affine algebraic variety of dimension $2 n$ [177,212], and it follows from $[19,135]$ that $\operatorname{Aut}(X)$ acts highly transitively on $X$ for every $n \geqslant 1$. Moreover, the variety $X$ is flexible by [5, Proposition 2.9].

\footnotetext{
${ }^{1}$ Or infinitely transitively in another terminology.
} 
There are several constructions producing new flexible varieties from given ones (see $[9,14,63,117])$. For instance, the product of flexible varieties is flexible. Some further examples of flexible varieties are as follows.

Example 4.6. Suppose that $X$ is an affine $G$-variety of dimension $\geqslant 2$, where $G$ is a connected linear algebraic group that acts on $X$ with an open orbit. Then $X$ is flexible in the following cases:

- $X$ is a normal toric variety with no torus factor [14, Theorem 0.2.2];

- $X=G / H$ is a homogeneous space and $G$ has no non-trivial character [9, Theorem 5.4];

- $\quad X$ is smooth and $G$ is semisimple [9, Theorem 5.6];

- $\quad X$ is smooth with only constant invertible functions and $G$ is reductive [84, Theorem 2];

- $\quad X$ is normal and $G=\mathrm{SL}_{2}(\mathbb{k})$ [9, Theorem 5.7];

- $\quad X$ is normal horospherical and $G$ is semisimple [201, Theorem 2];

- $X$ is normal horospherical with no non-constant invertible regular function [84, Theorem 3].

See also $[54,55,83,85,115,136]$.

If we replace the smooth locus of $X$ in Theorem 4.2 by the open orbit of the group $\operatorname{SAut}(X)$, we obtain a criterion for the generic flexibility [9]. If $X$ contains $\mathbb{A}^{n}$ as a principal Zariski open set, then $X$ is generically flexible. Generically flexible varieties are unirational, but they are not always stably rational (see [144, Proposition 4.9] and [176, Example 1.22]).

Example 4.7. Suppose that $X$ is a normal affine surface such that $X$ can be completed by a simple normal crossing chain of rational curves. Then $X$ is often called a Gizatullin surface. If $X \nsupseteq \mathbb{A}^{1} \times\left(\mathbb{A}^{1} \backslash\{0\}\right)$, then it is generically flexible [86], but it is not necessarily flexible [134].

Affine cones over cylindrical Fano varieties often provide examples of flexible affine varieties.

Example 4.8. Let $V=G / P$, where $G$ is a semisimple algebraic group, and $P$ is its parabolic subgroup. Then $V$ is a smooth Fano variety. Let $V \hookrightarrow \mathbb{P}^{n}$ be any projectively normal embedding, and let $\hat{V}$ be the affine cone in $\mathbb{A}^{n+1}$ over $V$. If $\operatorname{dim}(V) \geqslant 2$, then $\hat{V}$ is flexible by [14, Theorem 1.1].

To explain why this is the case, let us present two explicit criteria of flexibility of affine cones. To do this, fix a smooth projective variety $V$. Let $H$ be a very ample divisor on the variety $X$. Then the linear system $|H|$ gives an embedding $V \hookrightarrow \mathbb{P}^{n}$. Let $\widehat{V}$ be the affine cone in $\mathbb{A}^{n+1}$ over $V$. We are interested in the case when $V$ is a smooth cylindrical Fano variety. 
If the variety $V$ is uniformly cylindrical, then each point of $V$ is contained in a cylinder, so that the variety $V$ admits a covering

$$
V=\bigcup_{i \in I} U_{i},
$$

where each $U_{i}$ is a Zariski open subset in $V$ such that $U_{i} \cong \mathbb{A}^{1} \times Z_{i}$ for some affine variety $Z_{i}$. In this case, a subset $Y \subset V$ is said to be invariant with respect to a cylinder $U_{i}$ if

$$
Y \cap U_{i}=\pi_{i}^{-1}\left(\pi_{i}\left(Y \cap U_{i}\right)\right)
$$

where $\pi_{i}: U_{i} \rightarrow Z_{i}$ is the natural projection.

Definition 4.10. If $V$ is uniformly cylindrical, then we say that the covering (4.9) is transversal if no proper subset $Y \subset X$ is invariant with respect to every cylinder $U_{i}$ in the covering (4.9).

Now, we are ready to state the first flexibility criterion for affine cones.

Theorem 4.11 ([171]). Suppose that $V$ is uniformly cylindrical and has a covering (4.9) such that

(i) the covering (4.9) is transversal;

(ii) each cylinder in the covering (4.9) is $H$-polar.

Then the affine cone $\widehat{V}$ is flexible.

The second useful criterion is given by the following

Theorem 4.12 ([149]). The affine cone $\widehat{V}$ is flexible if the variety $V$ is uniformly cylindrical and admits a covering

$$
V=\bigcup_{j \in J} W_{j},
$$

where each $W_{j}$ is a flexible affine Zariski open subset in $V$ such that $W_{j}=V \backslash \operatorname{Supp} D_{j}$ for some effective $\mathbb{Q}$-divisor $D_{j}$ on the variety $V$ that satisfies $D_{j} \sim_{\mathbb{Q}} H$.

Using these criteria and the proof of Lemma 2.17, one can prove the following result:

Theorem 4.13 ([168, 171]). Suppose that $V$ is a smooth del Pezzo surface such that $K_{V}^{2} \geqslant 4$. Then the affine cone $\hat{V}$ is flexible for every very ample divisor $H$ on the surface $V$.

Unfortunately, we cannot apply Theorems 4.11 and 4.12 to the affine cone in $\mathbb{A}^{4}$ over a smooth cubic surface in $\mathbb{P}^{3}$, simply because its anticanonical divisor is not cylindrical by Corollary 2.9. On the other hand, in this case, we know from Theorem 2.14 that every ample $\mathbb{Q}$-divisor that is not a multiple of the anticanonical divisor is cylindrical. Using this and the construction of cylinders given in the proof of Theorem 2.14, Perepechko very recently proved the following result: 
Theorem 4.14 ([170]). If $V$ is a smooth cubic surface, then the affine cone $\widehat{V}$ is generically flexible for every very ample divisor $H$ on the surface $V$ such that $H \notin \mathbb{Z}_{>0}\left[-K_{V}\right]$.

Now, let us consider the flexibility of affine cones over some cylindrical smooth Fano threefolds. Many of them are flexible by Theorem 4.12, because the underlying Fano threefolds admit covering like in Theorem 4.12 with each Zariski open subset $W_{j}$ isomorphic to $\mathbb{A}^{3}$. A possibly non-complete list of such smooth Fano threefolds is given in [12, Proposition 4]. This gives the following corollary:

Corollary 4.15. Suppose $V$ is a smooth Fano threefold admitting an effective $\mathrm{PSL}_{2}(\mathbb{k})$ action. If $\rho(V)=1$, then the affine cone $\widehat{V}$ is flexible.

Proof. If $\rho(V)=1$, then it follows from Theorem 3.5 that one of the following four cases occurs:

(i) $\quad V=\mathbb{P}^{3}$;

(ii) $\quad V$ is the smooth quadric threefold in $\mathbb{P}^{4}$;

(iii) $V$ is the smooth quintic del Pezzo threefold $V_{5} \subset \mathbb{P}^{6}$ described in Example 1.31;

(iv) $V$ is the Mukai-Umemura threefold $X=X_{22}^{\mathrm{mu}} \subset \mathbb{P}^{13}$.

We may assume that we are in the case (iii) or (iv), because the required assertion is clear in the remaining cases. Then it follows from the proofs of Theorems 3.6 and 3.10 that $V$ contains a one-parameter family of hyperplane sections $H_{\ell}$ such that each $H_{\ell}$ is singular along a line $\ell$ and

$$
V \backslash H_{\ell} \cong \mathbb{A}^{3}
$$

The group $\mathrm{PSL}_{2}(\mathbb{k})$ acts transitively on this family. So, to apply Theorem 4.12, we need to check that the intersection of all these hyperplane sections is empty. Suppose that this is not the case. Then this intersection is $\mathrm{PSL}_{2}(\mathbb{k})$-invariant, so that it contains a closed $\mathrm{PSL}_{2}(\mathbb{k})$-orbit of minimal dimension. But the variety $V$ does not contain $\mathrm{PSL}_{2}(\mathbb{k})$-fixed points, and the only one-dimensional closed $\operatorname{PSL}_{2}(\mathbb{k})$-orbit in $V$ is not contained in any hyperplane section singular along a line.

For more examples of smooth Fano threefolds with flexible affine cones, see [149, Theorem 4.5]. Now, let us present examples of smooth Fano fourfolds with flexible affine cones.

Example 4.16 ([188]). It follows from Theorems 3.20 and 3.23 that the following smooth cylindrical Fano fourfolds admit coverings by affine charts isomorphic to $\mathbb{A}^{4}$ :

(1) the quintic del Pezzo fourfold $V_{5}$ described in Example 1.31 (see Theorem 3.20);

(2) the Fano-Mukai fourfold $X_{18}^{\mathrm{s}}$ of genus 10 with $\operatorname{Aut}\left(X_{18}^{\mathrm{s}}\right) \cong \mathrm{GL}_{2}(\mathbb{C}) \rtimes \mu_{2}$;

(3) the Fano-Mukai fourfolds $X_{18}$ of genus 10 with $\operatorname{Aut}^{0}\left(X_{18}\right) \cong \mathbb{G}_{\mathrm{m}}^{2}$ (there is a oneparameter family of these, up to isomorphism).

Hence, all of them have flexible affine cones. 
By Theorem 3.23, every smooth Fano-Mukai fourfold in $\mathbb{P}^{12}$ of genus 10 contains a Zariski open subset isomorphic to $\mathbb{A}^{4}$. Moreover, the following result has been recently proved in [185].

Theorem 4.17. The affine cones over any smooth Fano-Mukai fourfold of genus 10 are flexible.

For more higher-dimensional examples of flexible affine cones, see [149].

\subsection{Cylinders in complements to hypersurfaces}

This section is motivated by the following folklore conjecture, which first appeared in 2005 [66].

Conjecture 4.18. Let $S$ be a smooth cubic surface in $\mathbb{P}^{3}$. Then any automorphism of the affine variety $\mathbb{P}^{3} \backslash S$ is induced by an automorphism of $\mathbb{P}^{3}$, i.e., we have

$$
\operatorname{Aut}\left(\mathbb{P}^{3} \backslash S\right)=\operatorname{Aut}\left(\mathbb{P}^{3}, S\right)
$$

If $S$ is smooth surface in $\mathbb{P}^{3}$ of degree $\geqslant 4$, then it is easy to see that

$$
\operatorname{Aut}\left(\mathbb{P}^{3} \backslash S\right)=\operatorname{Aut}\left(\mathbb{P}^{3}, S\right) .
$$

Vice versa, if $S$ is either a smooth quadric surface or a plane in $\mathbb{P}^{3}$, then

$$
\operatorname{Aut}\left(\mathbb{P}^{3} \backslash S\right) \neq \operatorname{Aut}\left(\mathbb{P}^{3}, S\right) .
$$

Moreover, it is not hard to see that Conjecture 4.18 fails for some singular cubic surfaces.

Example 4.19. Let $S$ be one of the three cubic surfaces with Du Val singularities in $\mathbb{P}^{3}$ that admits an effective $\mathbb{G}_{\mathrm{a}}$-action (see $\left.[41,147,197]\right)$. Then $\operatorname{Aut}\left(\mathbb{P}^{3}, S\right)$ contains a subgroup isomorphic to $\mathbb{G}_{\mathrm{a}}$, so that $\operatorname{Aut}\left(\mathbb{P}^{3} \backslash S\right)$ also contains a subgroup isomorphic to $\mathbb{G}_{\mathrm{a}}$. Then Aut $\left(\mathbb{P}^{3} \backslash S\right)$ must be infinite dimensional (see [65]), so that $\operatorname{Aut}(\mathbb{P} \backslash S) \neq \operatorname{Aut}(\mathbb{P}, S)$, because $\operatorname{Aut}(\mathbb{P}, S)$ is algebraic.

Based on the results in $[33,38,39]$, we may generalize the problem to del Pezzo surfaces that are hypersurfaces in weighted projective spaces. To be precise, let $S$ be a del Pezzo surface that has at most Du Val singularities such that $K_{S}^{2} \leqslant 3$. Then we have one of the following three cases:

(1) $K_{S}^{2}=1$, and $S$ is a hypersurface of degree 6 in $\mathbb{P}(1,1,2,3)$;

(2) $K_{S}^{2}=2$, and $S$ is a hypersurface of degree 4 in $\mathbb{P}(1,1,1,2)$;

(3) $K_{S}^{2}=3$, and $S$ is a hypersurface of degree 3 in $\mathbb{P}^{3}$.

Denote by $\mathbb{P}$ the weighted projective space in these three cases: $\mathbb{P}(1,1,2,3), \mathbb{P}(1,1,1,2)$ or $\mathbb{P}^{3}$. Then, very surprisingly, we have the following result:

Theorem 4.20 ([33, 165]). The following three conditions are equivalent:

- the surface $S$ contains a $\left(-K_{S}\right)$-polar cylinder; 
- the complement $\mathbb{P} \backslash S$ is cylindrical;

- the group $\operatorname{Aut}(\mathbb{P} \backslash S)$ contains a unipotent subgroup.

Combining this result with Theorem 2.8, we obtain the following corollary.

Corollary 4.21 ([165, Corollary 1.6]). The group $\operatorname{Aut}(\mathbb{P} \backslash S)$ contains no unipotent subgroup exactly when $S$ is one of the surfaces listed in Theorem 2.8 .

Corollary 4.22 ([33, Corollary 4.10]). Suppose that the surface $S$ contains a $\left(-K_{S}\right)$ polar cylinder. Then $\operatorname{Aut}(\mathbb{P} \backslash S) \neq \operatorname{Aut}(\mathbb{P}, S)$.

Proof. By Theorem 4.20, the group $\operatorname{Aut}(\mathbb{P} \backslash S)$ contains a unipotent subgroup, so that it is infinite dimensional, which implies that $\operatorname{Aut}(\mathbb{P} \backslash S) \neq \operatorname{Aut}(\mathbb{P}, S)$, because $\operatorname{Aut}(\mathbb{P}, S)$ is algebraic.

This corollary together with Theorem 2.8 show that Conjecture 4.18 fails for all singular cubic surfaces that have Du Val singularities. On the other hand, we have:

Theorem 4.23 ([33, Theorem 4.1]). Suppose that $S$ is smooth. If $K_{S}^{2}=1$, then

$$
\text { Aut }(\mathbb{P} \backslash S)=\operatorname{Aut}(\mathbb{P}, S) \text {. }
$$

If $K_{S}^{2}=2$ or $K_{S}^{2}=3$, then $\operatorname{Aut}(\mathbb{P} \backslash S)$ does not contain non-trivial connected algebraic groups.

The proof of this result depends on irrationality of some del Pezzo threefolds (see [48, 87, 88, 210]). Taking into account Theorem 4.23, Corollary 2.9 and Corollary 4.22, we propose the following:

Conjecture 4.24. The surface $S$ contains no $\left(-K_{S}\right)$-polar cylinder $\Longleftrightarrow \operatorname{Aut}(\mathbb{P} \backslash S)=$ Aut $(\mathbb{P}, S)$.

If $S$ is a smooth cubic surface, then it does not contain any $\left(-K_{S}\right)$-polar cylinder by Theorem 2.8. In this case, Conjecture 4.24 claims that Aut $(\mathbb{P} \backslash S)=$ Aut $(\mathbb{P}, S)$, which is Conjecture 4.18.

In [165], Theorem 4.20 has been generalized as follows. Let $X$ be a normal projective variety, and let $D$ be an ample Cartier divisor on $X$. Suppose that the following conditions are satisfied:

(1) the section ring of $(X, D)$ is a hypersurface, i.e., one has

$$
\bigoplus_{m=0}^{\infty} \mathrm{H}^{0}\left(X, \mathcal{O}_{X}(m D)\right) \cong \mathbb{k}\left[x_{0}, x_{1}, \ldots, x_{n}\right] /(F),
$$

where $\mathbb{k}\left[x_{0}, \ldots, x_{n}\right]$ is a polynomial ring in variables $x_{0}, \ldots, x_{n}$ with weights

$$
a_{0}=\operatorname{wt}\left(x_{0}\right) \leqslant a_{1}=\operatorname{wt}\left(x_{1}\right) \leqslant \cdots \leqslant a_{n}=\operatorname{wt}\left(x_{n}\right),
$$


and $F$ is a quasi-homogeneous polynomial of degree $d$, so that $X$ is a hypersurface in the weighted projective space

$$
\mathbb{P}\left(a_{0}, a_{1}, \ldots, a_{n}\right)=\operatorname{Proj}\left(\mathbb{k}\left[x_{0}, x_{1}, \ldots, x_{n}\right]\right) ;
$$

(2) the Veronese map $v_{d}: \mathbb{P}\left(a_{0}, a_{1}, \ldots, a_{n}\right) \rightarrow \mathbb{P}^{N}$ given by $\left|\mathcal{O}_{\mathbb{P}\left(a_{0}, a_{1}, \ldots, a_{n}\right)}(d)\right|$ is an embedding.

Recall from [120, Proposition 3.5] that the complement $\mathbb{P}\left(a_{0}, a_{1}, \ldots, a_{n}\right) \backslash X$ admits a non-trivial $\mathbb{G}_{\mathrm{a}}$-action if and only if it is cylindrical. On the other hand, we have the following result:

Theorem 4.25 ([165, Theorem 3.1]). Suppose that $\mathbb{P}\left(a_{0}, a_{1}, \ldots, a_{n}\right) \backslash X$ has a nontrivial $\mathbb{G}_{\mathrm{a}}$-action. Then $X$ contains a D-polar cylinder.

Based on the results on non-ruledness of smooth hypersurfaces of low degrees in the projective spaces such as $[28,48,53,109,127,192,193,200]$ one can extend Conjecture 4.18 as follows:

Conjecture 4.26. Let $X$ be a smooth hypersurface in $\mathbb{P}^{n}$ of degree $d \geqslant 3$. Then

$$
\operatorname{Aut}\left(\mathbb{P}^{n} \backslash X\right)=\operatorname{Aut}\left(\mathbb{P}^{n}, X\right)
$$

The conjecture holds when $d>n$ since the hypersurface $X$ has non-negative Kodaira dimension. It remains true if $d=n \geqslant 4$ and $(n, d)=(4,3)$ due to the results by [28, 48, $53,109,192,193]$.

\subsection{Compactifications of $\mathbb{C}^{n}$}

In this subsection, we assume that varieties are defined over $\mathbb{C}$. In this case, the problem of existence of (Zariski open) cylinders in smooth Fano varieties is closely related to the following famous problem posed by Hirzebruch 65 years ago in [102].

Problem 4.27. Find all complex analytic compactifications of $\mathbb{C}^{n}$ with second Betti number 1 .

This problems asks to describe all compact complex manifolds $X$ with $\mathrm{b}_{2}(X)=1$ that contain an open subset $U$ which is biholomorphic to $\mathbb{C}^{n}$ and whose complement $A=X \backslash U$ is a closed complex analytic subspace. Thus, we call a compactification of $\mathbb{C}^{n}$ a pair $(X, A)$ consisting of

- a compact complex manifold $X$ with $\mathrm{b}_{2}(X)=1$;

- $\quad$ and a closed complex analytic subset $A \subset X$ such that $X \backslash A \underset{\text { bihol }}{\cong} \mathbb{C}^{n}$.

A compactification $(X, A)$ of $\mathbb{C}^{n}$ is said to be algebraic if $X$ is a smooth projective variety, and the biholomorphism $X \backslash A \underset{\text { bihol }}{\cong} \mathbb{C}^{n}$ is an algebraic isomorphism. Thus, we see that

$(X, A)$ is an algebraic compactification of $\mathbb{C}^{n} \Longrightarrow X$ is a cylindrical Fano variety. 
Proposition 4.28 ([25,208]). Let $(X, A)$ be a compactification of $\mathbb{C}^{n}$. Then the following hold:

(1) A is purely 1-codimensional and irreducible;

(2) $H^{i}(X, \mathbb{Z}) \cong H^{i}(A, \mathbb{Z}), H_{i}(X, \mathbb{Z}) \cong H_{i}(A, \mathbb{Z})$ for every $i \leqslant 2 n-2$;

(3) $H^{1}(X, \mathbb{Z})=0$ and $H_{1}(X, \mathbb{Z})=0$;

(4) the class of $A$ generates the groups $H^{2}(X, \mathbb{Z}) \cong \mathbb{Z}$ and $H^{2}(A, \mathbb{Z}) \cong \mathbb{Z}$;

(5) if $X$ is Moishezon, then $H^{1}\left(X, \mathcal{O}_{X}\right)=0$ and $H^{2}\left(X, \mathcal{O}_{X}\right)=0$, so that $\operatorname{Pic}(X) \cong$ $H^{2}(X, \mathbb{Z})$.

The following deep result is due to Kodaira [126, Theorem 3]:

Theorem 4.29. If $(X, A)$ is a compactification of $\mathbb{C}^{n}$, then

$$
h^{0}\left(X, \omega_{X}^{\otimes m}\right)=0
$$

for every $m>0$, where $\omega_{X}$ is the sheaf of holomorphic $n$-forms on $X$.

Thus, if $(X, A)$ is a compactification of $\mathbb{C}^{n}$ and $X$ is projective, then $X$ is a smooth Fano variety, and $A$ is an ample divisor on $X$ that generates $\operatorname{Pic}(X)$.

Example 4.30. Let $(X, A)$ be one of the following polarized smooth Fano varieties:

(1) $X=\mathbb{P}^{n}$ and $A$ is a hyperplane;

(2) $X$ is a smooth quadric in $\mathbb{P}^{n+1}$ and $A$ is its singular hyperplane section;

(3) $X=\operatorname{Gr}(m, k)$ and $A$ is its Schubert subvariety of codimension 1 , where $n=$ $m(k-m)$;

(4) $X=G / P$ and $A$ is its open cell isomorphic to $\mathbb{C}^{n}$ (such a cell does exist by [23, 128]), where $G$ is a semisimple connected complex linear algebraic group, and $P$ is its maximal parabolic subgroup.

Then $(X, A)$ is a compactification of $\mathbb{C}^{n}$.

In two-dimensional case, Problem 4.27 has an easy solution: if $(X, A)$ is a compactification of $\mathbb{C}^{2}$, then $X=\mathbb{P}^{2}$ and $A$ is a line in $X$. In the three-dimensional case, Problem 4.27 has been solved in the series of papers $[75-79,81,172,173,180]$. In particular, we have the following result:

Theorem 4.31. Let $(X, A)$ be a compactification of $\mathbb{C}^{3}$. Suppose that $X$ is a projective threefold. Then this compactification is algebraic and $(X, A)$ can be described as follows:

(1) $X=\mathbb{P}^{3}$ and $A$ is a plane;

(2) $X$ is a smooth quadric in $\mathbb{P}^{4}$ and $A$ is its singular hyperplane section;

(3) $X$ is the quintic del Pezzo threefold in $\mathbb{P}^{5}$ described in Example 1.31 and $A$ is its singular hyperplane section that can be described as follows: 
(a) a surface whose singular locus is a line L with normal bundle

$$
\mathcal{N}_{L / X}=\mathcal{O}_{L}(1) \oplus \mathcal{O}_{L}(-1)
$$

(b) a normal del Pezzo surface that has a unique singular point of type $\mathrm{A}_{4}$;

(4) $X$ is a smooth Fano threefold of index 1 and genus 12 in $\mathbb{P}^{13}$ and $A$ is its certain hyperplane section whose singular locus is a line $\ell$ with normal bundle

$$
\mathcal{N}_{\ell / X}=\mathcal{O}_{\ell}(1) \oplus \mathcal{O}_{\ell}(-2)
$$

Proof. We know that $X$ is a smooth Fano threefold, and the surface $A$ generates $\operatorname{Pic}(X)$, so that

$$
-K_{X} \sim \iota(X) A,
$$

where $\iota(X)$ is the Fano index of the threefold $X$. If $\iota(X)=4$, then $X=\mathbb{P}^{3}$ and $A$ is a plane. Similarly, if $\iota(X)=3$, then $X$ is a smooth quadric threefold in $\mathbb{P}^{4}$, and $A$ is its hyperplane section. In this case, the surface $A$ must be singular, since $H^{2}(A, Z)=\mathbb{Z}$ by Proposition 4.28.

If $\iota(X)=1$, then the surface $A$ must be a non-normal K3 surface, and the proof uses a delicate analysis of its singularities. As a result, one can show that $X$ is a Fano threefold of genus 12 in $\mathbb{P}^{13}$, and $A$ is its hyperplane section that is singular along a line of type $(1,-2)$. One construction of such compactification is described in Remark 3.12. We will not dwell into further details in this case.

Suppose that $\iota(X)=2$. Let us show that $X$ is the quintic del Pezzo threefold in $\mathbb{P}^{5}$, and $A$ is its singular hyperplane section described above. Note that in this case $(X, A)$ is indeed a compactification of $\mathbb{C}^{3}$, which follows from the proof of Theorem 3.6.

By Proposition 4.28 , we have $H^{2}(A, Z)=\mathbb{Z}$ and

$$
4+2 h^{1,2}(X)=\chi_{\text {top }}(X)=\chi_{\text {top }}(A)+1 .
$$

First, we suppose that the surface $A$ is normal. Then $-K_{A}$ is ample by the adjunction formula, so that $A$ is a del Pezzo surface with isolated Gorenstein singularities. If its singularities are worse than Du Val, then $A$ must be a (generalized) cone over an elliptic curve [101], so that $\chi_{\text {top }}(A)=1$. The latter contradicts (4.32). Thus, we see that $A$ is a del Pezzo surface with Du Val singularities. Then $\rho(A)=1$, because $H^{2}(A, Z)=\mathbb{Z}$. Then $\chi_{\text {top }}(A)=3$, so that we have $h^{1,2}(X)=0$ by (4.32). Now, using Remark 3.1, we conclude that $X$ is the quintic del Pezzo threefold in $\mathbb{P}^{5}$ as required. Moreover, we have $K_{A}^{2}=5$, so that $A$ is a quintic del Pezzo surface that has Du Val singularities. Since $\rho(A)=1$, it follows from [75,152] that $A$ has a unique singular point of type $\mathrm{A}_{4}$.

Now, we suppose that $A$ is non-normal, so that it has a singular locus of positive dimension. It is easy to show that any hyperplane section of a smooth complete intersection has only isolated singularities, and the same result holds for hyperplane sections of weighed smooth hypersurfaces. Therefore, using Remark 3.1, we conclude again that $X$ is the quintic del Pezzo threefold in $\mathbb{P}^{5}$, and $A$ is its hyperplane section. Using the adjunction 
formula, we see that a general hyperplane section of the surface $A$ is an irreducible singular curve of arithmetic genus 1, so that it has one singular point. Thus, the non-normal locus of the surface $A$ is some line $L$. Hence, it follows from the proof of Theorem 3.6 that $\operatorname{Sing}(A)=L$ and

$$
X \backslash A \cong Q \backslash H,
$$

where $Q$ is a smooth quadric threefold in $\mathbb{P}^{4}$, and $H$ is its hyperplane section. Since $X \backslash A \cong \mathbb{C}^{3}$, we conclude that the surface $H$ is singular. As we already mentioned in the proof of Theorem 3.6, this implies that $\mathcal{N}_{L / X}=\mathcal{O}_{L}(1) \oplus \mathcal{O}_{L}(-1)$ as required.

Corollary 4.33. Let $(X, A)$ be a compactification of $\mathbb{C}^{3}$. Suppose that $X$ is a projective threefold. Then $H^{k}(X, \mathbb{Z}) \cong H^{k}\left(\mathbb{P}^{3}, \mathbb{Z}\right)$ for all $k$.

It would be interesting to find an alternative proof of Theorem 4.31 that does not heavily rely on the classification of smooth Fano threefolds.

Remark 4.34. Let $X$ be a smooth Fano threefold such that $\rho(X)=1, \iota(X)=1$ and $\mathrm{g}(X)=12$. If $X$ is a compactification of $\mathbb{C}^{4}$, then $X$ contains a line $\ell$ such that $\mathcal{N}_{\ell / X}=$ $\mathcal{O}_{\ell}(1) \oplus \mathcal{O}_{\ell}(-2)$. However, this condition does not always guarantee that $X$ is a compactification of $\mathbb{C}^{4}$ (see [180]).

Remark 4.35. The list in Theorem 4.31 is similar to the list in Theorem 3.5.

In higher dimensions, we know very few results on Problem 4.27. Let us present one of them, which follows from Theorem 3.20 and its proof. We use here the notation introduced in Section 3.2.

Theorem 4.36 ([182]). Let $(X, A)$ be a compactification of $\mathbb{C}^{4}$, where $X$ is a smooth Fano fourfold. Suppose that $\iota(X)=3$. Then $X$ is the quintic del Pezzo fourfold in $\mathbb{P}^{7}$ and

(1) either $A=H_{\ell}$, where $\ell$ is a line in $X$ that is not a line of type (b);

(2) or $A$ is a singular hyperplane section of the del Pezzo fourfold $X$ such that its singular locus consists of a single ordinary double point that is not contained in the divisor $\mathscr{H}$.

Each of these compactifications is algebraic and unique up to isomorphism.

Proof. We prove the existence part only. In the first case, the existence follows from Theorem 3.20. To deal with the second case, let us use the notation introduced in the proof of Theorem 3.20. Consider the Sarkisov link (3.21) with $\ell$ being a line of type (a). We already know that $Q$ is smooth, and so we may assume that it is given in $\mathbb{P}^{4}$ by

$$
x_{2} x_{3}+x_{1} x_{4}+x_{0} x_{5}=0 .
$$


Similarly, we may assume that $\eta(F)$ is cut out by $x_{0}=0$. Moreover, the surface $\eta\left(\hat{H}_{\ell}\right)$ is a smooth cubic scroll in this case. Hence, we may assume that it is cut out on $Q$ by

$$
\left\{\begin{array}{l}
x_{0}=0 \\
x_{2} x_{4}+x_{1} x_{5}=0 \\
x_{4}^{2}-x_{3} x_{5}=0
\end{array}\right.
$$

Let $D$ be the hyperplane section of the quadric $Q$ that is cut out by $x_{3}=0$, and let $\widehat{D}$ be its proper transform on $\widehat{V}_{5}$. Then $D$ is singular. We claim that $\widehat{V}_{5} \backslash\left(\widehat{D} \cup \hat{H}_{\ell}\right) \cong \mathbb{A}^{4}$. Indeed, let $U=Q \backslash D$. Then $U \cong \mathbb{A}^{4}$ with coordinates

$$
y_{0}=\frac{x_{0}}{x_{3}}, \quad y_{1}=\frac{x_{1}}{x_{3}}, \quad y_{4}=\frac{x_{4}}{x_{3}}, \quad y_{5}=\frac{x_{5}}{x_{4}},
$$

so that $\widehat{V}_{5} \backslash \widehat{D}$ is given by

$$
y_{0} z_{0}=\left(y_{5}-y_{4}^{2}\right) z_{1}
$$

in $\mathbb{A}^{4} \times \mathbb{P}^{1}$, where $z_{0}$ and $z_{1}$ are coordinates on $\mathbb{P}^{1}$. Then $\hat{V}_{5} \backslash\left(\hat{D} \cup \hat{H}_{\ell}\right)$ is given in $\mathbb{A}^{4} \times \mathbb{A}^{1}$ by

$$
y_{0} z=y_{5}-y_{4}^{2},
$$

where $z=\frac{z_{0}}{z_{1}}$. This implies that $\hat{V}_{5} \backslash\left(\hat{D} \cup \hat{H}_{\ell}\right) \cong \mathbb{A}^{4}$. Now, observe that $\pi(\hat{D})$ is a hyperplane section of $V_{5}$ whose singular locus consists of a single ordinary double point not contained in $\mathscr{H}$.

In dimension 4 , we know very few compactifications $(X, A)$ of $\mathbb{C}^{4}$. They can be listed as follows:

- $\quad X=\mathbb{P}^{4}$ and $A$ is a hyperplane;

- $\quad X$ is a smooth quadric and $A$ is its singular hyperplane section;

- $\quad X$ is the del Pezzo quintic fourfold and $A$ is described in Theorem 4.36;

- $X$ is a smooth Fano-Mukai fourfold of genus 10 and $A$ is described in Theorem 3.23. In particular, in every known example of a compactification $(X, A)$ of $\mathbb{C}^{4}$ with $X \nsupseteq \mathbb{P}^{4}$, one has

$$
H^{k}(X, \mathbb{Z}) \cong H^{k}(Q, \mathbb{Z})
$$

for all $k$, where $Q$ is a smooth quadric in $\mathbb{P}^{5}$. We wonder whether this is just a coincidence.

Question 4.37. Does there exist a smooth Fano fourfold of index 1 that is a compactification of $\mathbb{C}^{4}$ ?

Before we conclude this survey, let us set the following question:

Question 4.38. Is it true that any compactification of $\mathbb{C}^{n}$ is rational?

Note that the answer to this question is not obvious, since the isomorphism $X \backslash A \cong \mathbb{C}^{n}$ in the definition of a compactification of $\mathbb{C}^{n}$ is a biholomorphism, which is not necessarily algebraic. 


\section{A. Singularities of pairs}

Let $S$ be a surface with at most quotient singularities, let $D$ be an effective non-zero $\mathbb{Q}$-divisor on $S$, let $P$ be a point of $S$, and let

$$
D=\sum_{i=1}^{r} a_{i} C_{i},
$$

where $C_{1}, \ldots, C_{r}$ are distinct irreducible curves on $S$, and each $a_{i}$ is a non-negative rational number. We call $(S, D)$ a $\log$ pair.

Let $\pi: \widetilde{S} \rightarrow S$ be a birational morphism such that $\widetilde{S}$ is smooth. For each $C_{i}$, denote by $\widetilde{C}_{i}$ its proper transform on the surface $\widetilde{S}$. Let $F_{1}, \ldots, F_{n}$ be $\pi$-exceptional curves. Then

$$
K_{\tilde{S}}+\sum_{i=1}^{r} a_{i} \widetilde{C}_{i}+\sum_{j=1}^{n} b_{j} F_{j} \sim_{\mathbb{Q}} \pi^{*}\left(K_{S}+D\right)
$$

for some rational numbers $b_{1}, \ldots, b_{n}$. Suppose that $\widetilde{C}_{1}+\cdots+\widetilde{C}_{2}+F_{1}+\cdots+F_{n}$ is a divisor with simple normal crossings. Then we say that $\pi: \widetilde{S} \rightarrow S$ is a log resolution of the log pair $(S, D)$.

Definition A.1. The log pair $(S, D)$ is said to be $\log$ canonical at the point $P$ if the following two conditions are satisfied:

- $a_{i} \leqslant 1$ for every $C_{i}$ such that $P \in C_{i}$;

- $b_{j} \leqslant 1$ for every $F_{j}$ such that $\pi\left(F_{j}\right)=P$.

The log pair $(S, D)$ is called $\log$ canonical if it is $\log$ canonical at every point of $S$.

This definition does not depend on the choice of the $\log$ resolution $\pi: \widetilde{S} \rightarrow S$.

Remark A.2. Let $R$ be an effective $\mathbb{Q}$-divisor on $S$ such that $R \sim_{\mathbb{Q}} D$. For a rational number $\epsilon$, let

$$
D_{\epsilon}=(1+\epsilon) D-\epsilon R \text {. }
$$

Then $D_{\epsilon} \sim_{\mathbb{Q}} D$. Suppose that $R \neq D$. Then there exists the greatest rational number $\epsilon_{0} \geqslant 0$ such that the divisor $D_{\epsilon_{0}}$ is effective. By construction, the support of the divisor $D_{\epsilon_{0}}$ does not contain at least one curve contained in the support of the divisor $R$. Moreover, if $(S, D)$ is not $\log$ canonical at $P$, but $(S, R)$ is $\log$ canonical at $P$, then $\left(S, D_{\epsilon_{0}}\right)$ is not $\log$ canonical at $P$.

Now, we suppose that the surface $S$ is smooth at $P$.

Lemma A.3. Suppose that $(S, D)$ is not log canonical at $P$. Then mult $P(D)>1$.

Proof. Left to the reader.

Let $f: \bar{S} \rightarrow S$ be a blowup of the point $P$, and let $E$ be the $f$-exceptional curve. Denote by $\bar{D}$ the proper transform of the $\mathbb{Q}$-divisor $D$ on the surface $\bar{S}$ via $f$. Then 
the log pair

$$
\left(\bar{S}, \bar{D}+\left(\operatorname{mult}_{P}(D)-1\right) E\right)
$$

is called the log pull back of the $\log$ pair $(S, D)$ on the surface $\bar{S}$.

Lemma A.5. Suppose that the log pair $(S, D)$ is not log canonical at $P$. Then

(i) the $\mathbb{Q}$-divisor $\bar{D}+\left(\right.$ mult $\left._{P}(D)-1\right) E$ is effective;

(ii) the log pair (A.4) is not log canonical at some point $Q \in E$.

Proof. The required assertion follows from Definition A.1 and Lemma A.3.

The following handy statement is a very special case of a much more general result, which is known as Inversion of Adjunction (see, for example, [131, Theorem 6.29]).

Lemma A.6 ([131, Exercise 6.31]). Suppose that $C_{1}$ is smooth at $P$, the log pair $(S, D)$ is not $\log$ canonical at $P$, and $a_{1} \leqslant 1$. Let $\Delta=a_{2} C_{2}+\cdots+a_{r} C_{r}$. Then $\left(C_{1} \cdot \Delta\right)_{P}>1$.

Proof. Let $m=\operatorname{mult}_{P}(\Delta)$. If $m>1$, then we are done, since

$$
\left(C_{1} \cdot \Delta\right)_{P} \geqslant m
$$

Therefore, we may assume that $m \leqslant 1$. This implies that the log pair $(S, D)$ is $\log$ canonical in a punctured neighborhood of the point $P \in S$. Since the $\log$ pair $(S, D)$ is not $\log$ canonical at $P$, there exists a birational morphism $h: \widehat{S} \rightarrow S$ that is a composition of $s \geqslant 1$ blowups of points dominating $P$ such that $e_{s}>1$, where $e_{s}$ is a rational number determined by

$$
K_{\widehat{S}}+a_{1} \widehat{C}_{1}+\widehat{\Delta}+\sum_{i=1}^{s} e_{i} E_{i} \sim_{\mathbb{Q}} h^{*}\left(K_{S}+D\right),
$$

where each $e_{i}$ is a rational number, each $E_{i}$ is an $h$-exceptional divisor, $\widehat{\Delta}$ is a proper transform on the surface $\widehat{S}$ of the divisor $\Delta$, and $\widehat{C}_{1}$ is a proper transform on $\widehat{S}$ of the curve $C_{1}$.

Let $\bar{\Delta}$ and $\bar{C}_{1}$ be the proper transforms on $\bar{S}$ of the divisor $\Delta$ and the curve $C_{1}$, respectively. Then $\left(\bar{S}, a_{1} \bar{C}_{1}+\left(a_{1}+m-1\right) E+\bar{\Delta}\right)$ is not log canonical at some point $Q \in E$ by Lemma A.5.

Let us prove the inequality $\left(C_{1} \cdot \Delta\right)_{P}>1$ by induction on $s$. If $s=1$, then

$$
a_{1}+m-1>1
$$

which implies that $m>2-a_{1} \geqslant 1$, so that $\left(C_{1} \cdot \Delta\right)_{P} \geqslant m>1$ as required. Thus, we may assume that $s \geqslant 2$ and $a_{1}+m-1 \leqslant 2$. Since

$$
\left(C_{1} \cdot \Delta\right)_{P} \geqslant m+\left(\bar{C}_{1} \cdot \bar{\Delta}\right)_{Q}
$$

it is enough to show that $m+\left(\bar{C}_{1} \cdot \bar{\Delta}\right)_{Q}>1$. We may also assume that $m \leqslant 1$, since $\left(C_{1} \cdot \Delta\right)_{P} \geqslant m$. 
If $Q \notin \bar{C}_{1}$, then $\left(\bar{S},\left(a_{1}+m-1\right) E+\bar{\Delta}\right)$ is not log canonical at the point $Q$, which gives

$$
m=\bar{\Delta} \cdot E \geqslant(\bar{\Delta} \cdot E)_{Q}>1
$$

by induction. The latter implies that $Q=\bar{C}_{1} \cap E$, since $m \leqslant 1$. Then

$$
a_{1}+m-1+\left(\bar{C}_{1} \cdot \bar{\Delta}\right)_{Q}=\left(\left(\left(a_{1}+m-1\right) E+\bar{\Delta}\right) \cdot \bar{C}_{1}\right)_{Q}>1
$$

by induction. This gives $(\bar{C} \cdot \bar{\Delta})_{Q}>2-a_{1}-m$. Then

$$
m+\left(\bar{C}_{1} \cdot \bar{\Delta}\right)_{Q}>2-a_{1} \geqslant 1
$$

as required.

Corollary A.7. In the notation and assumptions of Lemma A.5, suppose that

$$
\operatorname{mult}_{P}(D) \leqslant 2 \text {. }
$$

Then there exists a unique point $Q \in E$ such that (A.4) is not log canonical at $Q$.

Proof. If (A.4) is not log canonical at two distinct points $P_{1}$ and $P_{2}$, then

$$
2 \geqslant \operatorname{mult}_{P}(D)=\bar{D} \cdot E \geqslant(\bar{D} \cdot E)_{P_{1}}+(\bar{D} \cdot E)_{P_{2}}>2
$$

by Lemma A.6. Now use Lemma A.5.

The following result plays an essential role in the proof of Theorem 2.15 given in Section 2.2. In fact, this theorem has been discovered [32] in an attempt to give a simple proof of Theorem 2.15, since its original proof in [38] is very technical. For other applications of Theorem 2.15, see [1,209].

Theorem A.8 ([32]). Suppose that $\left(C_{1} \cdot C_{2}\right)_{P}=1$, and the log pair $(S, D)$ is not log canonical at $P$. Let $\Delta=a_{3} C_{3}+\cdots+a_{r} C_{r}$ and $m=$ mult $_{P}(\Delta)$. Suppose also that $m \leqslant 1$. Then

$$
\begin{array}{ll} 
& \left(C_{1} \cdot \Delta\right)_{P}>2\left(1-a_{2}\right) \\
\text { or } & \left(C_{2} \cdot \Delta\right)_{P}>2\left(1-a_{1}\right) .
\end{array}
$$

Proof. We may assume that $a_{1} \leqslant 1$ and $a_{2} \leqslant 1$. There is a morphism $h: \hat{S} \rightarrow S$ that is a composition of $s \geqslant 1$ blowups of points dominating $P$ such that $e_{s}>1$ for $e_{s} \in \mathbb{Q}$ that is determined by

$$
K_{\widehat{S}}+a_{1} \widehat{C}_{1}+a_{2} \widehat{C}_{2}+\widehat{\Delta}+\sum_{i=1}^{r} e_{i} E_{i}=h^{*}\left(K_{S}+a_{1} C_{1}+a_{2} C_{2}+\Delta\right),
$$

where each $e_{i}$ is a rational number, each $E_{i}$ is an $h$-exceptional divisor, $\widehat{C}_{1}$ and $\widehat{C}_{2}$, are proper transforms on $\widehat{S}$ of the curves $C_{1}$ and $C_{2}$, respectively, and $\widehat{\Delta}$ is a proper transform of the divisor $\Delta$. 
Let $\bar{\Delta}, \bar{C}_{1}, \bar{C}_{2}$ be the proper transforms on $\bar{S}$ of the divisors $\Delta, C_{1}$ and $C_{2}$, respectively. Then

$$
\left(\bar{S}, a_{1} \bar{C}_{1}+a_{2} \bar{C}_{2}+\left(a_{1}+a_{2}+m-1\right) E+\bar{\Delta}\right)
$$

is not $\log$ canonical at some point $Q \in E$ by Lemma A.5.

If $s=1$, then $a_{1}+a_{2}+m-1>1$. If $m>2-a_{1}-a_{2}$, then $m>2\left(1-a_{1}\right)$ or $m>$ $2\left(1-a_{2}\right)$, because otherwise we would have

$$
2 m \leqslant 4-2\left(a_{1}+a_{2}\right),
$$

which contradicts to $m>2-a_{1}-a_{2}$. Then

or

$$
\begin{aligned}
& \left(\Delta \cdot C_{1}\right)_{P}>2\left(1-a_{2}\right) \\
& \left(\Delta \cdot C_{2}\right)_{P}>2\left(1-a_{1}\right)
\end{aligned}
$$

if $s=1$.

Let us prove the required assertion by induction on $s$. The case $s=1$ is already done, so that we may assume that $s \geqslant 2$ and $a_{1}+a_{2}+m \leqslant 2$. If $Q \neq E \cap \bar{C}_{1}$ and $Q \neq E \cap \bar{C}_{2}$, then

$$
m=\bar{\Delta} \cdot E>1
$$

by Lemma A.6, which is impossible by assumption. Thus, either $Q=E \cap \bar{C}_{1}$ or $Q=$ $E \cap \bar{C}_{2}$. Without loss of generality, we may assume that $Q=E \cap \bar{C}_{1}$.

By induction, we can apply the lemma to $\left(\bar{S}, a_{1} \bar{C}_{1}+\left(a_{1}+a_{2}+m-1\right) E+\bar{\Delta}\right)$ at the point $Q$. This implies that either

$$
\left(\bar{\Delta} \cdot \bar{C}_{1}\right)_{Q}>2\left(1-\left(a_{1}+a_{2}+m-1\right)\right)=4-2 a_{1}-2 a_{2}-2 m
$$

or $(\bar{\Delta} \cdot E)_{Q}>2\left(1-a_{1}\right)$. In the latter case, we have

$$
\left(\Delta \cdot C_{2}\right)_{P} \geqslant m=\bar{\Delta} \cdot E \geqslant(\bar{\Delta} \cdot E)_{Q}>2\left(1-a_{1}\right),
$$

which is exactly what we want. Therefore, we may assume that

$$
\left(\bar{\Delta} \cdot \bar{C}_{1}\right)_{Q}>4-2 a_{1}-2 a_{2}-2 m .
$$

If $\left(\Delta \cdot C_{2}\right)_{P}>2\left(1-a_{1}\right)$, then we are done. Hence, we may assume $\left(\Delta \cdot C_{2}\right)_{P} \leqslant 2\left(1-a_{1}\right)$. Then

$$
m \leqslant\left(\Delta \cdot C_{2}\right)_{P} \leqslant 2\left(1-a_{1}\right)
$$

This gives

$$
\left(\Delta \cdot C_{1}\right)_{P} \geqslant m+\left(\bar{\Delta} \cdot \bar{C}_{1}\right)_{Q}>m+4-2 a_{1}-2 a_{2}-2 m>2\left(1-a_{2}\right),
$$

because $m \leqslant 2\left(1-a_{1}\right)$.

Almost all results we have considered so far in this subsection are local (except for Remark A.2). Let us conclude this subsection by two global statements. The first of them is due to Puhklikov: 
Lemma A.9 ([131, Lemma 5.36]). Suppose that $S$ is a smooth surface in $\mathbb{P}^{3}$, and $D$ is $\mathbb{Q}$-linearly equivalent to its hyperplane section. Then each $a_{i}$ does not exceed 1.

Proof. Let $X$ be a cone over the curve $C_{i}$ whose vertex is a general enough point in $\mathbb{P}^{3}$. Then

$$
X \cap S=C_{i}+\widehat{C}_{i}
$$

where $\hat{C}_{i}$ is an irreducible curve of degree $(\operatorname{deg}(S)-1) \operatorname{deg}\left(C_{i}\right)$. Moreover, $\widehat{C}_{i}$ is not contained in the support of the divisor $D$, and the intersection $C_{i} \cap \widehat{C}_{i}$ consists of exactly $\operatorname{deg}\left(\widehat{C}_{i}\right)$ points. Then

$$
\operatorname{deg}\left(\widehat{C}_{i}\right)=D \cdot \widehat{C}_{i} \geqslant a_{i} C_{i} \cdot \widehat{C}_{i} \geqslant a_{i} \operatorname{deg}\left(\widehat{C}_{i}\right),
$$

which implies that $a_{i} \leqslant 1$.

The second global result we want to mention is the following lemma about del Pezzo surfaces of degree 2 that have at most two ordinary double points.

Lemma A.10. Suppose that there is a double cover $\tau: S \rightarrow \mathbb{P}^{2}$ branched over an irreducible quartic curve $B$ that has at most two ordinary double points, and

$$
D \sim \mathbb{Q}-K_{S}
$$

Then each $a_{i}$ does not exceed 1. Moreover, if $(S, D)$ is not log canonical at $P$, then $\tau(P) \in B$.

Proof. Write $D=a_{1} C_{1}+\Delta$, where $\Delta=a_{2} C_{2}+\cdots+a_{r} C_{r}$. Suppose that $a_{1}>1$. Let us seek for a contradiction. Since

$$
\begin{aligned}
2=-K_{S} \cdot D & =-K_{S} \cdot\left(a_{1} C_{1}+\Delta\right) \\
& =-a_{1} K_{S} \cdot C_{1}-K_{S} \cdot \Delta \geqslant-a_{1} K_{S} \cdot C_{1}>-K_{S} \cdot C_{1},
\end{aligned}
$$

we have $-K_{S} \cdot C_{1}=1$. Then $\tau\left(C_{1}\right)$ is a line. Hence, the surface $S$ contains an irreducible curve $Z_{1}$ such that $C_{1}+Z_{1} \sim-K_{S}$ and $\tau\left(C_{1}\right)=\tau\left(Z_{1}\right)$. Note that the curves $C_{1}$ and $Z_{1}$ are interchanged by the biregular involution of the surface $S$ induced by the double cover $\tau$. Then

$$
2=\left(-K_{S}\right)^{2}=\left(C_{1}+Z_{1}\right)^{2}=2 C_{1}^{2}+2 C_{1} \cdot Z_{1},
$$

which implies that $C_{1} \cdot Z_{1}=1-C_{1}^{2}$. Since $C_{1}$ and $Z_{1}$ are smooth rational curves, we have

$$
C_{1}^{2}=Z_{1}^{2}=-1+\frac{k}{2}
$$

where $k$ is the number of singular points of $S$ that lie on $C_{1}$. Now we write

$$
D=a_{1} C_{1}+b_{1} Z_{1}+\Theta
$$


where $b_{1}$ is a non-negative rational number, and $\Theta$ is an effective $\mathbb{Q}$-divisor whose support does not contains the curves $C_{1}$ and $Z_{1}$. Then

$$
1=C_{1} \cdot\left(a_{1} C_{1}+b_{1} Z_{1}+\Theta\right) \geqslant a_{1} C_{1}^{2}+b_{1} C_{1} \cdot Z_{1}=a_{1} C_{1}^{2}+b_{1}\left(1-C_{1}^{2}\right),
$$

and hence $1 \geqslant a_{1} C_{1}^{2}+b_{1}\left(1-C_{1}^{2}\right)$. Similarly, from $Z_{1} \cdot D=1$, we obtain

$$
1 \geqslant b_{1} C_{1}^{2}+a_{1}\left(1-C_{1}^{2}\right) \text {. }
$$

The obtained two inequalities imply that $a_{1} \leqslant 1$ and $b_{1} \leqslant 1$, because $C_{1}^{2}=-1+\frac{k}{2}$ and $k \leqslant 2$. Since $a_{1}>1$ by our assumption, this is a contradiction.

We see that $a_{1} \leqslant 1$. Similarly, we see that $a_{i} \leqslant 1$ for every $i$.

Now we suppose that the $\log$ pair $(S, D)$ is not $\log$ canonical at $P$. Let us show that $\tau(P) \in B$. Suppose that $\tau(P) \notin B$. Then $S$ is smooth at $P$. Let us seek for a contradiction.

Let $H$ be a general curve in $\left|-K_{S}\right|$ that passes through the point $P$. Then

$$
2=H \cdot D \geqslant \operatorname{mult}_{P}(H) \operatorname{mult}_{P}(D) \geqslant \operatorname{mult}_{P}(D),
$$

so that $\operatorname{mult}_{P}(D) \leqslant 2$. But the pair (A.4) is not $\log$ canonical at some point $Q \in E$ by Lemma A.5. Applying Lemma A.3 to (A.4), we get $\operatorname{mult}_{P}(D)+\operatorname{mult}_{Q}(\bar{D})>2$.

Since $\tau(P) \notin B$, there exists a unique (possibly reducible) curve $R \in\left|-K_{S}\right|$ such that its proper transform on $\bar{S}$ passes through the point $Q$. Note that $R$ is smooth at $P$. This enables us to assume that the support of $D$ does not contain at least one irreducible component of $R$ by Remark A.2. Denote by $\bar{R}$ the proper transform of $R$ on the surface $\bar{R}$. If the curve $R$ is irreducible, then

$$
\begin{aligned}
2-\operatorname{mult}_{P}(D) & =2-\operatorname{mult}_{P}(C) \operatorname{mult}_{P}(D) \\
& =\bar{R} \cdot \bar{D} \geqslant \operatorname{mult}_{Q}(\bar{R}) \operatorname{mult}_{Q}(\bar{D})=\operatorname{mult}_{Q}(\bar{D}),
\end{aligned}
$$

which is impossible, since mult mo $_{P}(D)+$ mult $_{Q}(\bar{D})>2$. Thus, the curve $R$ must be reducible.

Write $R=R_{1}+R_{2}$, where $R_{1}$ and $R_{2}$ are irreducible smooth curves. Without loss of generality we may assume that the curve $R_{1}$ is not contained in $\operatorname{Supp}(D)$. Then $P \in R_{2}$, because otherwise we would have

$$
1=D \cdot R_{1} \geqslant \operatorname{mult}_{P}(D)>1,
$$

since $\operatorname{mult}_{P}(D)>1$ by Lemma A.3. Thus, we put $D=a R_{2}+\Omega$, where $a$ is a nonnegative rational number and $\Omega$ is an effective $\mathbb{Q}$-divisor whose support does not contain the curve $R_{2}$. Then

$$
1=R_{1} \cdot D=\left(2-\frac{1}{2} l\right) a+R_{1} \cdot \Omega \geqslant\left(2-\frac{1}{2} l\right) a,
$$

where $l$ is the number of singular points of the surface $S$ contained in $R_{1}$. Denote by $\bar{R}_{2}$ the proper transform on $\bar{S}$ of the curve $R_{2}$, and denote by $\bar{\Omega}$ the proper transform on $\bar{S}$ of the divisor $\Omega$. Then the $\log$ pair

$$
\left(\bar{S}, a \bar{R}_{2}+\bar{\Omega}+\left(\operatorname{mult}_{P}(D)-1\right) E\right)
$$


is not $\log$ canonical at $Q$. Note that we already proved that $a \leqslant 1$. Thus, using Lemma A.6, we get

$$
\left(2-\frac{1}{2} l\right) a=\bar{R}_{2} \cdot\left(\bar{\Omega}+\left(\operatorname{mult}_{P}(D)-1\right) E\right)>1 .
$$

This is a contradiction.

Acknowledgments. We would like to thank Adrien Dubouloz and Sasha Perepechko for useful comments.

Funding. The first and the third authors were partially supported by the HSE University Basic Research Program, and by the Royal Society grant No. IES\R1\180205. The first author is partially supported by Laboratory of Mirror Symmetry NRU HSE, RF Government grant, ag. No 14.641.31.0001. The second author has been supported by IBSR003-D1, Institute for Basic Science in Korea.

\section{References}

[1] H. Ahmadinezhad, I. Cheltsov, and J. Schicho, On a conjecture of Tian. Math. Z. 288 (2018), no. 1-2, 217-241 Zbl 1390.14109 MR 3774411

[2] J. Alper, H. Blum, D. Halpern-Leistner, and C. Xu, Reductivity of the automorphism group of K-polystable Fano varieties. Invent. Math. 222 (2020), no. 3, 995-1032 Zbl 07269010 MR 4169054

[3] T. Ando, On extremal rays of the higher-dimensional varieties. Invent. Math. 81 (1985), no. 2, 347-357 Zbl 0554.14001 MR 799271

[4] M. Andreatta and J. A. Wiśniewski, On contractions of smooth varieties. J. Algebraic Geom. 7 (1998), no. 2, 253-312 Zbl 0966.14012 MR 1620110

[5] R. Andrist, The density property for Calogero-Moser spaces, 2020 arXiv:2006.11936

[6] M. Artin and D. Mumford, Some elementary examples of unirational varieties which are not rational. Proc. London Math. Soc. (3) 25 (1972), 75-95 Zbl 0244.14017 MR 321934

[7] I. Arzhantsev, On rigidity of factorial trinomial hypersurfaces. Internat. J. Algebra Comput. 26 (2016), no. 5, 1061-1070 Zbl 1350.13008 MR 3536446

[8] I. Arzhantsev, Polynomial curves on trinomial hypersurfaces. Acta Arith. 186 (2018), no. 1, 87-99 Zbl 06970438 MR 3865680

[9] I. Arzhantsev, H. Flenner, S. Kaliman, F. Kutzschebauch, and M. Zaidenberg, Flexible varieties and automorphism groups. Duke Math. J. 162 (2013), no. 4, 767-823 Zbl 1295.14057 MR 3039680

[10] I. Arzhantsev and S. Gaifullin, The automorphism group of a rigid affine variety. Math. Nachr. 290 (2017), no. 5-6, 662-671 Zbl 1386.14156 MR 3636369

[11] I. Arzhantsev, K. Kuyumzhiyan, and M. Zaidenberg, Infinite transitivity, finite generation, and Demazure roots. Adv. Math. 351 (2019), 1-32 Zbl 1428.14092 MR 3949984

[12] I. Arzhantsev, A. Perepechko, and H. Süß, Infinite transitivity on universal torsors. J. Lond. Math. Soc. (2) 89 (2014), no. 3, 762-778 Zbl 1342.14105 MR 3217648

[13] I. Arzhantsev and M. Zaidenberg, Tits-type alternative for groups acting on toric affine varieties, to appear in Int. Math. Res. Not. DOI: 10.1093/imrn/rnaa342 
[14] I. V. Arzhantsev, M. G. Ză̌denberg, and K. G. Kuyumzhiyan, Flag varieties, toric varieties, and suspensions: three examples of infinite transitivity. Mat. Sb. 203 (2012), no. 7, 3-30 Zbl 1311.14059 MR 2986429

[15] V. V. Batyrev and Y. I. Manin, Sur le nombre des points rationnels de hauteur borné des variétés algébriques. Math. Ann. 286 (1990), no. 1-3, 27-43 Zbl 0679.14008 MR 1032922

[16] A. Beauville, Variétés de Prym et jacobiennes intermédiaires. Ann. Sci. École Norm. Sup. (4) 10 (1977), no. 3, 309-391 Zbl 0368.14018 MR 472843

[17] G. Belousov, Cylinders in del Pezzo surfaces with du Val singularities. Bull. Korean Math. Soc. 54 (2017), no. 5, 1655-1667 Zbl 1398.14040 MR 3708802

[18] O. Benoist and O. Wittenberg, Intermediate jacobians and rationality over arbitrary fields, 2019 arXiv: 1909.12668

[19] Y. Berest, A. Eshmatov, and F. Eshmatov, Multitransitivity of Calogero-Moser spaces. Transform. Groups 21 (2016), no. 1, 35-50 Zbl 1337.16017 MR 3459703

[20] G. Bodnár, H. Hauser, J. Schicho, and O. Villamayor U., Plain varieties. Bull. Lond. Math. Soc. 40 (2008), no. 6, 965-971 Zbl 1159.14007 MR 2471945

[21] F. Bogomolov and C. Böhning, On uniformly rational varieties. In Topology, geometry, integrable systems, and mathematical physics, pp. 33-48, Amer. Math. Soc. Transl. Ser. 2234 , Amer. Math. Soc., Providence, RI, 2014 Zbl 1360.14043 MR 3307142

[22] A. Borel, Les bouts des espaces homogènes de groupes de Lie. Ann. of Math. (2) 58 (1953), 443-457 Zbl 0053.13002 MR 57263

[23] A. Borel, Kählerian coset spaces of semisimple Lie groups. Proc. Nat. Acad. Sci. U.S.A. 40 (1954), 1147-1151 Zbl 0058.16002 MR 77878

[24] V. Borovik and S. Gaifullin, $m$-suspensions over rigid varieties, 2020 arXiv:2007.07882

[25] L. Brenton and J. Morrow, Compactifications of $\mathbf{C}^{n}$. Trans. Amer. Math. Soc. 246 (1978), 139-153 Zbl 0416.32015 MR 515533

[26] F. Campana, Connexité rationnelle des variétés de Fano. Ann. Sci. École Norm. Sup. (4) 25 (1992), no. 5, 539-545 Zbl 0783.14022 MR 1191735

[27] A.-M. Castravet, Examples of Fano varieties of index one that are not birationally rigid. Proc. Amer. Math. Soc. 135 (2007), no. 12, 3783-3788 Zbl 1130.14015 MR 2341927

[28] I. A. Chel'tsov, On a smooth four-dimensional quintic. Mat. Sb. 191 (2000), no. 9, 139-160 Zbl 0998.14018 MR 1805602

[29] I. A. Chel'tsov and K. A. Shramov, Log canonical thresholds of smooth Fano threefolds. Russ. Math. Surv. 63 (2008), no. 5, 859-958 Zbl 1167.14024 MR 2484031

[30] I. A. Chel'tsov and K. A. Shramov, Extremal metrics on del Pezzo threefolds. Proc. Steklov Inst. Math. 264 (2009), 37-51 Zbl 1312.14095 MR 2590832

[31] I. Cheltsov, Log canonical thresholds of del Pezzo surfaces. Geom. Funct. Anal. 18 (2008), no. 4, 1118-1144 Zbl 1161.14030 MR 2465686

[32] I. Cheltsov, Del Pezzo surfaces and local inequalities. In Automorphisms in birational and affine geometry, pp. 83-101, Springer Proc. Math. Stat. 79, Springer, Cham, 2014 Zbl 1327.14174 MR 3229346

[33] I. Cheltsov, A. Dubouloz, and J. Park, Super-rigid affine Fano varieties. Compos. Math. 154 (2018), no. 11, 2462-2484 Zbl 1408.14052 MR 3866510

[34] I. Cheltsov and D. Kosta, Computing $\alpha$-invariants of singular del Pezzo surfaces. J. Geom. Anal. 24 (2014), no. 2, 798-842 Zbl 1309.14031 MR 3192299

[35] I. Cheltsov and J. Park, Sextic double solids. In Cohomological and geometric approaches to rationality problems, pp. 75-132, Progr. Math. 282, Birkhäuser Boston, Boston, MA, 2010 Zbl 1200.14031 MR 2605166 
[36] I. Cheltsov, J. Park, and C. Shramov, Exceptional del Pezzo hypersurfaces. J. Geom. Anal. 20 (2010), no. 4, 787-816 Zbl 1211.14047 MR 2683768

[37] I. Cheltsov, J. Park, and J. Won, Log canonical thresholds of certain Fano hypersurfaces. Math. Z. 276 (2014), no. 1-2, 51-79 Zbl 1288.14031 MR 3150192

[38] I. Cheltsov, J. Park, and J. Won, Affine cones over smooth cubic surfaces. J. Eur. Math. Soc. (JEMS) 18 (2016), no. 7, 1537-1564 Zbl 1386.14068 MR 3506607

[39] I. Cheltsov, J. Park, and J. Won, Cylinders in singular del Pezzo surfaces. Compos. Math. 152 (2016), no. 6, 1198-1224 Zbl 1360.14020 MR 3518309

[40] I. Cheltsov, J. Park, and J. Won, Cylinders in del Pezzo surfaces. Int. Math. Res. Not. IMRN (2017), no. 4, 1179-1230 Zbl 1405.14089 MR 3658164

[41] I. Cheltsov and Y. Prokhorov, Del Pezzo surfaces with infinite automorphism groups. Algebr. Geom. 8 (2021), no. 3, 319-357 Zbl 1456.14051 MR 4206439

[42] I. Cheltsov, V. Przyjalkowski, and C. Shramov, Which quartic double solids are rational? J. Algebraic Geom. 28 (2019), no. 2, 201-243 Zbl 1430.14032 MR 3912057

[43] I. Cheltsov and C. Shramov, Del Pezzo zoo. Exp. Math. 22 (2013), no. 3, 313-326 Zbl 1281.14034 MR 3171095

[44] I. Cheltsov and C. Shramov, Cremona groups and the icosahedron. Monogr. Res. Notes Math., CRC Press, Boca Raton, FL, 2016 Zbl 1328.14003 MR 3444095

[45] I. A. Cheltsov, Cylinders in rational surfaces. Mat. Sb. 212 (2021), no. 3, 139-156 Zbl 07349636 MR 4223975

[46] M. Chitayat and D. Daigle, Locally nilpotent derivations of graded integral domains and cylindricity, 2021 arXiv:2105.01729

[47] M. Chitayat and D. Daigle, On the rigidity of certain Pham-Brieskorn rings. J. Algebra 550 (2020), 290-308 Zbl 07167051 MR 4056984

[48] C. H. Clemens and P. A. Griffiths, The intermediate Jacobian of the cubic threefold. Ann. of Math. (2) 95 (1972), 281-356 Zbl 0214.48302 MR 302652

[49] A. Corti, Singularities of linear systems and 3-fold birational geometry. In Explicit birational geometry of 3-folds, pp. 259-312, London Math. Soc. Lecture Note Ser. 281, Cambridge Univ. Press, Cambridge, 2000 Zbl 0960.14017 MR 1798984

[50] S. Cynk, Defect of a nodal hypersurface. Manuscripta Math. 104 (2001), no. 3, 325-331 Zbl 0983.14017 MR 1828878

[51] D. Daigle, G. Freudenburg, and L. Moser-Jauslin, Locally nilpotent derivations of rings graded by an abelian group. In Algebraic varieties and automorphism groups, pp. 29-48, Adv. Stud. Pure Math. 75, Math. Soc. Japan, Tokyo, 2017 Zbl 1396.14060 MR 3793361

[52] T. de Fernex, Negative curves on very general blow-ups of $\mathbb{P}^{2}$. In Projective varieties with unexpected properties, pp. 199-207, Walter de Gruyter, Berlin, 2005 Zbl 1121.14006 MR 2202253

[53] T. de Fernex, Birationally rigid hypersurfaces. Invent. Math. 192 (2013), no. 3, 533-566 Zbl 1279.14019 MR 3049929

[54] A. Dubouloz, Flexible bundles over rigid affine surfaces. Comment. Math. Helv. 90 (2015), no. 1, 121-137 Zbl 1326.14145 MR 3317335

[55] A. Dubouloz, Exotic $\mathbb{G}_{a}$-quotients of $\mathrm{SL}_{2} \times \mathbb{A}^{1}$. Eur. J. Math. 5 (2019), no. 3, 828-844 Zbl 07116156 MR 3993265

[56] A. Dubouloz and T. Kishimoto, Log-uniruled affine varieties without cylinder-like open subsets. Bull. Soc. Math. France 143 (2015), no. 2, 383-401 Zbl 1327.14196 MR 3351185

[57] A. Dubouloz and T. Kishimoto, Families of affine ruled surfaces: existence of cylinders. Nagoya Math. J. 223 (2016), no. 1, 1-20 Zbl 1362.14065 MR 3572739 
[58] A. Dubouloz and T. Kishimoto, Explicit biregular/birational geometry of affine threefolds: completions of $\mathbb{A}^{3}$ into del Pezzo fibrations and Mori conic bundles. In Algebraic varieties and automorphism groups, pp. 49-71, Adv. Stud. Pure Math. 75, Math. Soc. Japan, Tokyo, 2017 Zbl 1396.14017 MR 3793362

[59] A. Dubouloz and T. Kishimoto, Cylinders in del Pezzo fibrations. Israel J. Math. 225 (2018), no. 2, 797-815 Zbl 06898294 MR 3805666

[60] A. Dubouloz and T. Kishimoto, Cylindres dans les fibrations de Mori: formes du volume quintique de del Pezzo. Ann. Inst. Fourier (Grenoble) 69 (2019), no. 6, 2377-2393 Zbl 1445.14027 MR 4033922

[61] A. Dubouloz and T. Kishimoto, Deformations of $\mathbb{A}^{1}$-cylindrical varieties. Math. Ann. 373 (2019), no. 3-4, 1135-1149 Zbl 07062596 MR 3953123

[62] A. Dubouloz, L. Moser-Jauslin, and P.-M. Poloni, Inequivalent embeddings of the KorasRussell cubic 3-fold. Michigan Math. J. 59 (2010), no. 3, 679-694 Zbl 1213.14118 MR 2745757

[63] H. Flenner, S. Kaliman, and M. Zaidenberg, A Gromov-Winkelmann type theorem for flexible varieties. J. Eur. Math. Soc. (JEMS) 18 (2016), no. 11, 2483-2510 Zbl 1400.14145 MR 3562349

[64] H. Flenner and M. Zaidenberg, Rational curves and rational singularities. Math. Z. 244 (2003), no. 3, 549-575 Zbl 1043.14008 MR 1992024

[65] G. Freudenburg, Algebraic theory of locally nilpotent derivations. Second edn., Encyclopaedia Math. Sci. 136, Springer, Berlin, 2017 Zbl 1391.13001 MR 3700208

[66] G. Freudenburg and P. Russell, Open problems in affine algebraic geometry. In Affine algebraic geometry, pp. 1-30, Contemp. Math. 369, Amer. Math. Soc., Providence, RI, 2005 Zbl 1070.14528 MR 2126651

[67] K. Fujita, Examples of K-unstable Fano manifolds with the Picard number 1. Proc. Edinb. Math. Soc. (2) 60 (2017), no. 4, 881-891 Zbl 1386.14152 MR 3715691

[68] T. Fujita, On the structure of polarized manifolds with total deficiency one. I. J. Math. Soc. Japan 32 (1980), no. 4, 709-725 Zbl 0474.14017 MR 589109

[69] T. Fujita, On the structure of polarized manifolds with total deficiency one. II. J. Math. Soc. Japan 33 (1981), no. 3, 415-434 Zbl 0474.14018 MR 620281

[70] T. Fujita, On the structure of polarized manifolds with total deficiency one. III. J. Math. Soc. Japan 36 (1984), no. 1, 75-89 Zbl 0541.14036 MR 723595

[71] T. Fujita, On polarized manifolds whose adjoint bundles are not semipositive. In Algebraic geometry, Sendai, 1985, pp. 167-178, Adv. Stud. Pure Math. 10, North-Holland, Amsterdam, 1987 Zbl 0659.14002 MR 946238

[72] T. Fujita, On Kodaira energy and adjoint reduction of polarized manifolds. Manuscripta Math. 76 (1992), no. 1, 59-84 Zbl 0766.14027 MR 1171156

[73] T. Fujita, On Kodaira energy of polarized log varieties. J. Math. Soc. Japan 48 (1996), no. 1, 1-12 Zbl 0914.14001 MR 1361544

[74] T. Fujita, On Kodaira energy and adjoint reduction of polarized threefolds. Manuscripta Math. 94 (1997), no. 2, 211-229 Zbl 0969.14001 MR 1473897

[75] M. Furushima, Singular del Pezzo surfaces and analytic compactifications of 3-dimensional complex affine space $\mathbf{C}^{3}$. Nagoya Math. J. 104 (1986), 1-28 Zbl 0612.14037 MR 868434

[76] M. Furushima, Complex analytic compactifications of $\mathbf{C}^{3}$. Algebraic geometry (Berlin, 1988). Compositio Math. 76 (1990), no. 1-2, 163-196. Zbl 0721.32012 MR 1078861

[77] M. Furushima, Mukai-Umemura's example of the Fano threefold with genus 12 as a compactification of $\mathbf{C}^{3}$. Nagoya Math. J. 127 (1992), 145-165 Zbl 0792.14021 MR 1183657 
[78] M. Furushima, The complete classification of compactifications of $\mathbf{C}^{3}$ which are projective manifolds with the second Betti number one. Math. Ann. 297 (1993), no. 4, 627-662 Zbl 0788.32022 MR 1245410

[79] M. Furushima, A new example of a compactification of $\mathbf{C}^{3}$. Math. Z. 212 (1993), no. 3, 395-399 Zbl 0790.32027 MR 1207300

[80] M. Furushima and N. Nakayama, The family of lines on the Fano threefold $V_{5}$. Nagoya Math. J. 116 (1989), 111-122 Zbl 0731.14025 MR 1029973

[81] M. Furushima and N. Nakayama, A new construction of a compactification of $\mathbf{C}^{3}$. Tohoku Math. J. (2) 41 (1989), no. 4, 543-560 Zbl 0703.14025 MR 1025321

[82] S. Gaifullin, On rigidity of trinomial hypersurfaces and factorial trinomial varieties, 2019 arXiv:1902.06136

[83] S. Gaifullin, Automorphisms of Danielewski varieties. J. Algebra 573 (2021), 364-392 Zbl 07327762 MR 4203537

[84] S. Gaifullin and A. Shafarevich, Flexibility of normal affine horospherical varieties. Proc. Amer. Math. Soc. 147 (2019), no. 8, 3317-3330 Zbl 1420.14133 MR 3981110

[85] M. Gizatullin, Two examples of affine homogeneous varieties. Eur. J. Math. 4 (2018), no. 3, 1035-1064 Zbl 1436.14105 MR 3851128

[86] M. H. Gizatullin, Quasihomogeneous affine surfaces. Math. USSR Izv. 5 (1971), 1057-1081 Zbl 0249.14010 MR 0286791

[87] M. M. Grinenko, On the double cone over the Veronese surface. Izv. Ross. Akad. Nauk Ser. Mat. 67 (2003), no. 3, 5-22 Zbl 1082.14015 MR 1992191

[88] M. M. Grinenko, Mori structures on a Fano threefold of index 2 and degree 1. Tr. Mat. Inst. Steklova 246 (2004), 116-141 Zbl 1120.14031 MR 2101287

[89] M. Gromov, Oka's principle for holomorphic sections of elliptic bundles. J. Amer. Math. Soc. 2 (1989), no. 4, 851-897 Zbl 0686.32012 MR 1001851

[90] R. V. Gurjar, K. Masuda, and M. Miyanishi, Deformations of $\mathbb{A}^{1}$-fibrations. In Automorphisms in birational and affine geometry, pp. 327-361, Springer Proc. Math. Stat. 79, Springer, Cham, 2014 Zbl 1326.14146 MR 3229360

[91] R. V. Gurjar, K. Masuda, M. Miyanishi, and P. Russell, Affine lines on affine surfaces and the Makar-Limanov invariant. Canad. J. Math. 60 (2008), no. 1, 109-139 Zbl 1137.14049 MR 2381169

[92] R. V. Gurjar and M. Miyanishi, Automorphisms of affine surfaces with $\mathbb{A}^{1}$-fibrations. Michigan Math. J. 53 (2005), no. 1, 33-55 Zbl 1084.14062 MR 2125532

[93] R. V. Gurjar and D.-Q. Zhang, $\pi_{1}$ of smooth points of a log del Pezzo surface is finite. I. $J$. Math. Sci. Univ. Tokyo 1 (1994), no. 1, 137-180 Zbl 0841.14017 MR 1298542

[94] N. T. A. Hang, M. Hoff, and H. L. Truong, On cylindrical smooth rational Fano fourfolds, 2021 arXiv:2101.04441

[95] B. Hassett, Some rational cubic fourfolds. J. Algebraic Geom. 8 (1999), no. 1, 103-114 Zbl 0961.14029 MR 1658216

[96] B. Hassett, Cubic fourfolds, K3 surfaces, and rationality questions. In Rationality problems in algebraic geometry, pp. 29-66, Lecture Notes in Math. 2172, Springer, Cham, 2016 Zbl 1454.14111 MR 3618665

[97] B. Hassett, A. Pirutka, and Y. Tschinkel, A very general quartic double fourfold is not stably rational. Algebr. Geom. 6 (2019), no. 1, 64-75 Zbl 07020392 MR 3904799

[98] B. Hassett, S. Tanimoto, and Y. Tschinkel, Balanced line bundles and equivariant compactifications of homogeneous spaces. Int. Math. Res. Not. IMRN (2015), no. 15, 6375-6410 Zbl 1354.14075 MR 3384482 
[99] B. Hassett and Y. Tschinkel, Rationality of Fano threefolds of degree 18 over nonclosed fields, 2019 arXiv:1910.13816

[100] B. Hassett and Y. Tschinkel, On stable rationality of Fano threefolds and del Pezzo fibrations. J. Reine Angew. Math. 751 (2019), 275-287 Zbl 07062937 MR 3956696

[101] F. Hidaka and K. Watanabe, Normal Gorenstein surfaces with ample anti-canonical divisor. Tokyo J. Math. 4 (1981), no. 2, 319-330 Zbl 0496.14023 MR 646042

[102] F. Hirzebruch, Some problems on differentiable and complex manifolds. Ann. of Math. (2) 60 (1954), 213-236 Zbl 0056.16803 MR 66013

[103] D. Hwang and J. Keum, Construction of singular rational surfaces of Picard number one with ample canonical divisor. Proc. Amer. Math. Soc. 140 (2012), no. 6, 1865-1879 Zbl 1243.14027 MR 2888175

[104] A. Iliev, L. Katzarkov, and V. Przyjalkowski, Double solids, categories and non-rationality. Proc. Edinb. Math. Soc. (2) 57 (2014), no. 1, 145-173 Zbl 1303.14026 MR 3165018

[105] V. A. Iskovskikh, Fano 3-folds. I. Math. of the USSR, Izv. 11 (1977), 485-527 Zbl 0382.14013 MR 463151

[106] V. A. Iskovskikh, Fano 3-folds. II, Math. of the USSR, Izv. 12 (1978), 469-506 Zbl 0424.14012 MR 503430

[107] V. A. Iskovskih, Anticanonical models of three-dimensional algebraic varieties. J. Soviet Math. 13 (1980), 745-814 Zbl 0428.14016 MR 537685

[108] V. A. Iskovskih, Birational automorphisms of three-dimensional algebraic varieties. J. Soviet Math. 13 (1980), 815-868 Zbl 0428.14017 MR 537686

[109] V. A. Iskovskih and J. I. Manin, Three-dimensional quartics and counterexamples to the Lüroth problem. Mat. Sb. (N.S.) 86(128) (1971), 140-166 Zbl 0222.14009 MR 0291172

[110] V. A. Iskovskikh, Double projection from a line onto Fano 3-folds of the first kind. Math. USSR, Sb. 66 (1990), 265-284 Zbl 0691.14027 MR 993458

[111] V. A. Iskovskikh, Factorization of birational mappings of rational surfaces from the point of view of Mori theory. Russ. Math. Surveys 51 (1996), 585-652 Zbl 0914.14005 MR 1422227

[112] V. A. Iskovskikh, On the rationality problem for algebraic threefolds. Tr. Mat. Inst. Steklova 218 (1997), 190-232 Zbl 0930.14026 MR 1642381

[113] V. A. Iskovskikh and Y. G. Prokhorov, Fano varieties. In Algebraic geometry, V, pp. 1-247, Encyclopaedia Math. Sci. 47, Springer, Berlin, 1999 Zbl 0912.14013 MR 1668579

[114] V. A. Iskovskikh and A. V. Pukhlikov, Birational automorphisms of multidimensional algebraic manifolds. Contemp. Math. Appl. 19 (1994), 3-76

[115] S. Kaliman, Surfaces with big automorphism groups. In Functional analysis and geometry: Selim Grigorievich Krein centennial, pp. 185-196, Contemp. Math. 733, Amer. Math. Soc., Providence, RI, 2019 MR 3985276

[116] S. Kaliman, The Kraft-Russell generic equivalence theorem and its application. Michigan Math. J. 69 (2020), no. 4, 751-764 Zbl 07306319 MR 4168784

[117] S. Kaliman and M. Zaidenberg, Affine modifications and affine hypersurfaces with a very transitive automorphism group. Transform. Groups 4 (1999), no. 1, 53-95 Zbl 0956.14041 MR 1669174

[118] S. Kaliman and M. Zaidenberg, Miyanishi's characterization of the affine 3-space does not hold in higher dimensions. Ann. Inst. Fourier (Grenoble) 50 (2000), no. 6, 1649-1669 (2001) Zbl 0971.14044 MR 1817379

[119] S. Keel and J. McKernan, Rational curves on quasi-projective surfaces. Mem. Amer. Math. Soc. 140 (1999), no. 669, viii+153pp Zbl 0955.14031 MR 1610249 
[120] T. Kishimoto, Y. Prokhorov, and M. Zaidenberg, Group actions on affine cones. In Affine algebraic geometry, pp. 123-163, CRM Proc. Lecture Notes 54, Amer. Math. Soc., Providence, RI, 2011 MR 2768637

[121] T. Kishimoto, Y. Prokhorov, and M. Zaidenberg, $\mathbb{G}_{\mathrm{a}}$-actions on affine cones. Transform. Groups 18 (2013), no. 4, 1137-1153 Zbl 1297.14061 MR 3127989

[122] T. Kishimoto, Y. Prokhorov, and M. Zaidenberg, Affine cones over Fano threefolds and additive group actions. Osaka J. Math. 51 (2014), no. 4, 1093-1112 Zbl 1308.14066 MR 3273879

[123] T. Kishimoto, Y. Prokhorov, and M. Zaidenberg, Unipotent group actions on del Pezzo cones. Algebr. Geom. 1 (2014), no. 1, 46-56 Zbl 1418.14016 MR 3234113

[124] F. Knop, Mehrfach transitive Operationen algebraischer Gruppen. Arch. Math. (Basel) 41 (1983), no. 5, 438-446 Zbl 0557.14028 MR 731620

[125] S. Kobayashi and T. Ochiai, Characterizations of complex projective spaces and hyperquadrics. J. Math. Kyoto Univ. 13 (1973), 31-47 Zbl 0261.32013 MR 316745

[126] K. Kodaira, Holomorphic mappings of polydiscs into compact complex manifolds. J. Differential Geometry 6 (1971/72), 33-46 Zbl 0227.32008 MR 301228

[127] J. Kollár, Nonrational hypersurfaces. J. Amer. Math. Soc. 8 (1995), no. 1, 241-249 Zbl 0839.14031 MR 1273416

[128] J. Kollár, Rational curves on algebraic varieties. Ergeb. Math. Grenzgeb. (3) 32, Springer, Berlin, 1996 MR 1440180

[129] J. Kollár, Is there a topological Bogomolov-Miyaoka-Yau inequality? Pure Appl. Math. Q. 4 (2008), no. 2, Special Issue: In honor of Fedor Bogomolov. Part 1, 203-236 Zbl 1145.14031 MR 2400877

[130] J. Kollár, Y. Miyaoka, and S. Mori, Rational curves on Fano varieties. In Classification of irregular varieties (Trento, 1990), pp. 100-105, Lecture Notes in Math. 1515, Springer, Berlin, 1992 Zbl 0776.14012 MR 1180339

[131] J. Kollár, K. E. Smith, and A. Corti, Rational and nearly rational varieties. Cambridge Studies in Advanced Mathematics 92, Cambridge University Press, Cambridge, 2004 Zbl 1060.14073 MR 2062787

[132] M. Koras and P. Russell, Contractible threefolds and $\mathbf{C}^{*}$-actions on $\mathbf{C}^{3}$. J. Algebraic Geom. 6 (1997), no. 4, 671-695 Zbl 0882.14013 MR 1487230

[133] S. J. Kovács and Z. Patakfalvi, Projectivity of the moduli space of stable log-varieties and subadditivity of log-Kodaira dimension. J. Amer. Math. Soc. 30 (2017), no. 4, 959-1021 Zbl 1393.14034 MR 3671934

[134] S. Kovalenko, Transitivity of automorphism groups of Gizatullin surfaces. Int. Math. Res. Not. IMRN (2015), no. 21, 11433-11484 Zbl 1375.14205 MR 3456050

[135] K. Kuyumzhiyan, Infinite transitivity for Calogero-Moser spaces. Proc. Amer. Math. Soc. 148 (2020), no. 9, 3723-3731 Zbl 1454.14151 MR 4127820

[136] K. Kuyumzhiyan and F. Mangolte, Infinitely transitive actions on real affine suspensions. $J$. Pure Appl. Algebra 216 (2012), no. 10, 2106-2112 Zbl 1294.14020 MR 2925804

[137] A. Kuznetsov and Y. Prokhorov, Rationality of Fano threefolds over non-closed fields. To appear in Amer. J. Math.

[138] A. Kuznetsov and Y. Prokhorov, Rationality of Mukai varieties over non-closed fields. Progr. in Math. 342 (2021), 249-290

[139] A. Kuznetsov and Y. Prokhorov, Prime Fano threefolds of genus 12 with a $\mathbb{G}_{\mathrm{m}}$-action and their automorphisms. Épijournal Géom. Algébrique 2 (2018), Art. 3, 14pp Zbl 1411.14046 MR 3816899 
[140] A. G. Kuznetsov, Y. G. Prokhorov, and C. A. Shramov, Hilbert schemes of lines and conics and automorphism groups of Fano threefolds. Jpn. J. Math. 13 (2018), no. 1, 109-185 Zbl 1406.14031 MR 3776469

[141] A. Kuznetsova, Sextic double solids with Artin-Mumford obstructions to rationality, 2019 arXiv: 1901.05055

[142] Y. Lee and N. Nakayama, Simply connected surfaces of general type in positive characteristic via deformation theory. Proc. Lond. Math. Soc. (3) 106 (2013), no. 2, 225-286 Zbl 1267.14047 MR 3021462

[143] Y. Lee and J. Park, A simply connected surface of general type with $p_{g}=0$ and $K^{2}=2$. Invent. Math. 170 (2007), no. 3, 483-505 Zbl 1126.14049 MR 2357500

[144] A. Liendo, Affine $\mathbb{T}$-varieties of complexity one and locally nilpotent derivations. Transform. Groups 15 (2010), no. 2, 389-425 Zbl 1209.14050 MR 2657447

[145] A. Liendo and C. Petitjean, Uniformly rational varieties with torus action. Transform. Groups 24 (2019), no. 1, 149-153 Zbl 1418.32017 MR 3916093

[146] L. Marquand and J. Won, Cylinders in rational surfaces. Eur. J. Math. 4 (2018), no. 3, 11611196 Zbl 1440.14175 MR 3851133

[147] G. Martin and C. Stadlmayr, Weak del Pezzo surfaces with global vector fields, 2020 arXiv:2007.03665

[148] M. Mella, Birational geometry of quartic 3-folds. II. The importance of being $\mathbb{Q}$-factorial. Math. Ann. 330 (2004), no. 1, 107-126 Zbl 1058.14022 MR 2091681

[149] M. Michałek, A. Perepechko, and H. Süß, Flexible affine cones and flexible coverings. Math. Z. 290 (2018), no. 3-4, 1457-1478 Zbl 1408.14199 MR 3856860

[150] M. Miyanishi, Open algebraic surfaces. CRM Monogr. Ser. 12, Amer. Math. Soc., Providence, RI, 2001 Zbl 0964.14030 MR 1800276

[151] M. Miyanishi and T. Sugie, Affine surfaces containing cylinderlike open sets. J. Math. Kyoto Univ. 20 (1980), no. 1, 11-42 Zbl 0445.14017 MR 564667

[152] M. Miyanishi and D.-Q. Zhang, Gorenstein log del Pezzo surfaces of rank one. J. Algebra 118 (1988), no. 1, 63-84 Zbl 0664.14019 MR 961326

[153] S. Mori and S. Mukai, Classification of Fano 3-folds with $B_{2} \geq 2$. Manuscripta Math. 36 (1981/82), no. 2, 147-162 Zbl 0478.14033 MR 641971

[154] S. Mori and S. Mukai, Erratum: "Classification of Fano 3-folds with $B_{2} \geq 2$ " [Manuscripta Math. 36 (1981/82), no. 2, 147-162]. Manuscripta Math., 110 (2003), no. 3, p. 407 MR 1969009

[155] S. Mori and S. Mukai, On Fano 3-folds with $B_{2} \geq 2$. In Algebraic varieties and analytic varieties (Tokyo, 1981), pp. 101-129, Adv. Stud. Pure Math. 1, North-Holland, Amsterdam, 1983 MR 715648

[156] S. Mori and S. Mukai, Classification of Fano 3-folds with $B_{2} \geq 2$. I. In Algebraic and topological theories (Kinosaki, 1984), pp. 496-545, Kinokuniya, Tokyo, 1986 Zbl 0800.14021 MR 1102273

[157] D. R. Morrison, The birational geometry of surfaces with rational double points. Math. Ann. 271 (1985), no. 3, 415-438 Zbl 0539.14008 MR 787190

[158] S. Mukai, Curves, $K 3$ surfaces and Fano 3-folds of genus $\leq 10$. In Algebraic geometry and commutative algebra, Vol. I, pp. 357-377, Kinokuniya, Tokyo, 1988 Zbl 0701.14044 MR 977768

[159] S. Mukai, Biregular classification of Fano 3-folds and Fano manifolds of coindex 3. Proc. Nat. Acad. Sci. U.S.A. 86 (1989), no. 9, 3000-3002 Zbl 0679.14020 MR 995400 
[160] S. Mukai and H. Umemura, Minimal rational threefolds. In Algebraic geometry (Tokyo/Kyoto, 1982), pp. 490-518, Lecture Notes in Math. 1016, Springer, Berlin, 1983 Zbl 0526.14006 MR 726439

[161] Y. Odaka, C. Spotti, and S. Sun, Compact moduli spaces of del Pezzo surfaces and KählerEinstein metrics. J. Differential Geom. 102 (2016), no. 1, 127-172 Zbl 1344.58008 MR 3447088

[162] K. Oguiso and T. T. Truong, Explicit examples of rational and Calabi-Yau threefolds with primitive automorphisms of positive entropy. J. Math. Sci. Univ. Tokyo 22 (2015), no. 1, 361385 Zbl 1349.14055 MR 3329200

[163] K. Oguiso and D.-Q. Zhang, On the most algebraic $K 3$ surfaces and the most extremal log Enriques surfaces. Amer. J. Math. 118 (1996), no. 6, 1277-1297 Zbl 0889.14016 MR 1420924

[164] K. Oguiso and D.-Q. Zhang, On the complete classification of extremal log Enriques surfaces. Math. Z. 231 (1999), no. 1, 23-50 Zbl 0958.14029 MR 1696755

[165] J. Park, $\mathbf{G}_{a}$-actions on the complements of hypersurfaces, to appear in Transform. Groups. DOI: doi:10.1007/s00031-020-09589-x

[166] H. Park, J. Park, and D. Shin, A simply connected surface of general type with $p_{g}=0$ and $K^{2}=$ 3. Geom. Topol. 13 (2009), no. 2, 743-767 Zbl 1181.14042 MR 2469529

[167] H. Park, J. Park, and D. Shin, A simply connected surface of general type with $p_{g}=0$ and $K^{2}=4$. Geom. Topol. 13 (2009), no. 3, 1483-1494 Zbl 1181.14043 MR 2496050

[168] J. Park and J. Won, Flexible affine cones over del Pezzo surfaces of degree 4. Eur. J. Math. 2 (2016), no. 1, 304-318 Zbl 1371.14040 MR 3454104

[169] J. Park and J. Won, K-stability of smooth del Pezzo surfaces. Math. Ann. 372 (2018), no. 3-4, 1239-1276 Zbl 1404.14042 MR 3880298

[170] A. Perepechko, Affine cones over cubic surfaces are flexible in codimension one. Forum Math. 33 (2021), no. 2, 339-348 MR 4223068

[171] A. Y. Perepechko, Flexibility of affine cones over del Pezzo surfaces of degree 4 and 5. Funct. Anal. Appl., 47 (2013), 284-289 Zbl 1312.14099 MR 3185123

[172] T. Peternell, Compactifications of $\mathbf{C}^{3}$. II. Math. Ann. 283 (1989), no. 1, 121-137 Zbl 0671.14020 MR 973807

[173] T. Peternell and M. Schneider, Compactifications of $\mathbf{C}^{3}$. I. Math. Ann. 280 (1988), no. 1, 129-146 Zbl 0651.14025 MR 928301

[174] C. Petitjean, Equivariantly uniformly rational varieties. Michigan Math. J. 66 (2017), no. 2, 245-268 Zbl 1372.14042 MR 3657217

[175] J. Piontkowski and A. Van de Ven, The automorphism group of linear sections of the Grassmannians G(1, N). Doc. Math. 4 (1999), 623-664 Zbl 0934.14032 MR 1719726

[176] V. L. Popov, On the Makar-Limanov, Derksen invariants, and finite automorphism groups of algebraic varieties. In Affine algebraic geometry, pp. 289-311, CRM Proc. Lecture Notes 54, Amer. Math. Soc., Providence, RI, 2011 Zbl 1242.14044 MR 2768646

[177] V. L. Popov, On infinite dimensional algebraic transformation groups. Transform. Groups 19 (2014), no. 2, 549-568 Zbl 1309.14036 MR 3200435

[178] Y. Prokhorov, Geometrical properties of Fano threefolds (Russian). PhD thesis, Moscow State Univ., 1990

[179] Y. Prokhorov, Automorphism groups of Fano 3-folds, Russian Math. Surv. 45 (1990), 222 223 Zbl 0707.14037 MR 1071944

[180] Y. Prokhorov, Fano threefolds of genus 12 and compactifications of $\mathbb{C}^{3}$, St. Petersbg. Math. J. 3 (1991), 855-864 Zbl 0790.14038 MR 1152607 
[181] Y. Prokhorov, Rationality constructions of some Fano fourfolds of index 2. Moscow Univ. Math. Bull. 48 (1993), 32-35 MR 1223982

[182] Y. Prokhorov, Compactifications of $\mathbf{C}^{4}$ of index 3. In Algebraic geometry and its applications (Yaroslavl', 1992), pp. 159-169, Aspects Math., E25, Friedr. Vieweg, Braunschweig, 1994 Zbl 0816.32021 MR 1282026

[183] Y. Prokhorov, On the number of singular points of terminal factorial Fano threefolds. Math. Notes 101 (2017), no. 6, 1068-1073 Zbl 1391.14082 MR 3659567

[184] Y. Prokhorov, Russian Math. Surv. 73 (2018), no. 3, 375-456 Zbl 1400.14040 MR 3807895

[185] Y. Prokhorov and M. Zaidenberg, Affine cones over Fano-Mukai fourfolds of genus 10 are flexible, 2020 arXiv:2005.12092

[186] Y. Prokhorov and M. Zaidenberg, Examples of cylindrical Fano fourfolds. Eur. J. Math. 2 (2016), no. 1, 262-282 Zbl 1375.14206 MR 3454101

[187] Y. Prokhorov and M. Zaidenberg, New examples of cylindrical Fano fourfolds. In Algebraic varieties and automorphism groups, pp. 443-463, Adv. Stud. Pure Math. 75, Math. Soc. Japan, Tokyo, 2017 Zbl 1396.14062 MR 3793372

[188] Y. Prokhorov and M. Zaidenberg, Fano-Mukai fourfolds of genus 10 as compactifications of $\mathbb{C}^{4}$. Eur. J. Math. 4 (2018), no. 3, 1197-1263 Zbl 1423.14244 MR 3851134

[189] V. V. Przhiyalkovskiu, I. A. Chel'tsov, and K. A. Shramov, Fano threefolds with infinite automorphism groups. Izv. Math. 83 (2019), no. 4, 860-907 Zbl 1444.14074 MR 3985696

[190] V. V. Przhiyalkovskil and K. A. Shramov, Double quadrics with large automorphism groups. Proc. Steklov Inst. Math. 294 (2016), 154-175 Zbl 1375.14146 MR 3628499

[191] A. Pukhlikov, Birationally rigid varieties. Math. Surveys Monogr. 190, Amer. Math. Soc., Providence, RI, 2013 Zbl 1297.14001 MR 3060242

[192] A. V. Pukhlikov, Birational isomorphisms of four-dimensional quintics. Invent. Math. 87 (1987), no. 2, 303-329 Zbl 0613.14011 MR 870730

[193] A. V. Pukhlikov, Birational automorphisms of Fano hypersurfaces. Invent. Math. 134 (1998), no. 2, 401-426 Zbl 0964.14011 MR 1650332

[194] A. V. Pukhlikov, Birationally rigid Fano varieties. In The Fano Conference, pp. 659-681, Univ. Torino, Turin, 2004 Zbl 1072.14050 MR 2112597

[195] A. V. Pukhlikov, Birational geometry of Fano direct products. Izv. Math. 69 (2005), no. 6, 1225-1255 Zbl 1119.14011 MR 2190093

[196] F. Russo and G. Stagliano, Explicit rationality of some cubic fourfolds, 2018 arXiv: 1811.03502

[197] Y. Sakamaki, Automorphism groups on normal singular cubic surfaces with no parameters. Trans. Amer. Math. Soc. 362 (2010), no. 5, 2641-2666 Zbl 1200.14080 MR 2584614

[198] M. Sawahara, Cylinders in weak del Pezzo fibrations, 2019 arXiv:1912.09016

[199] M. Sawahara, Cylinders in canonical del Pezzo fibrations, 2020 arXiv:2012.10062

[200] S. Schreieder, Stably irrational hypersurfaces of small slopes. J. Amer. Math. Soc. 32 (2019), no. 4, 1171-1199 Zbl 1442.14138 MR 4013741

[201] A. A. Shafarevich, Flexibility of $S$-varieties of semisimple groups. Mat. $S b .208$ (2017), no. 2, 121-148 MR 3608041

[202] V. V. Shokurov, Prym varieties: theory and applications. Izv. Akad. Nauk SSSR Ser. Mat. 47 (1983), no. 4, 785-855 MR 712095

[203] C. Shramov, Birational rigidity and $\mathbb{Q}$-factoriality of a singular double quadric, 2007 arXiv:math/0701522

[204] K. Takeuchi, Some birational maps of Fano 3-folds. Compositio Math. 71 (1989), no. 3, 265283 Zbl 0712.14025 MR 1022045 
[205] G. Tian, On Kähler-Einstein metrics on certain Kähler manifolds with $C_{1}(M)>0$. Invent. Math. 89 (1987), no. 2, 225-246 Zbl 0599.53046 MR 894378

[206] G. Tian, On Calabi's conjecture for complex surfaces with positive first Chern class. Invent. Math. 101 (1990), no. 1, 101-172 Zbl 0716.32019 MR 1055713

[207] S. L. Tregub, Three constructions of rationality of a cubic fourfold. Vestnik Moskov. Univ. Ser. I Mat. Mekh. (1984), no. 3, 8-14 Zbl 0573.14021 MR 749015

[208] A. van de Ven, Analytic compactifications of complex homology cells. Math. Ann. 147 (1962), 189-204 Zbl 0105.14406 MR 140125

[209] N. Viswanathan, Lowest log canonical thresholds of a reduced plane curve of degree $d$. Eur. J. Math. 6 (2020), no. 4, 1216-1235 Zbl 7335103 MR 4183036

[210] C. Voisin, Sur la jacobienne intermédiaire du double solide d'indice deux. Duke Math. J. 57 (1988), no. 2, 629-646 Zbl 0698.14049 MR 962523

[211] F. Wang, The classification of log Enriques surfaces of rank 18. J. Korean Math. Soc. 48 (2011), no. 4, 797-822 Zbl 1228.14032 MR 2840525

[212] G. Wilson, Collisions of Calogero-Moser particles and an adelic Grassmannian. Invent. Math. 133 (1998), no. 1, 1-41 Zbl 0906.35089 MR 1626461

[213] C. Xu, K-stability of Fano varieties: an algebro-geometric approach. EMS Surv. Math. Sci. 8 (2021), no. 1, 265-354

[214] Q. Ye, On Gorenstein log del Pezzo surfaces. Japan. J. Math. (N.S.) 28 (2002), no. 1, 87-136 Zbl 1053.14044 MR 1933881

[215] Q. Zhang, Rational connectedness of $\log$ Q-Fano varieties. J. Reine Angew. Math. 590 (2006), 131-142 Zbl 1093.14059 MR 2208131

[216] Z. Zhuang, Optimal destabilizing centers and equivariant K-stability, 2020 arXiv:2004.09413

Received 8 May 2021.

\section{Ivan Cheltsov}

Department of Mathematics, University of Edinburgh, Edinburgh EH9 3JZ, UK;

National Research University Higher School of Economics, 6 Usacheva Street, Moscow 119048,

Russia; i.cheltsov@ed.ac.uk

\section{Jihun Park}

Center for Geometry and Physics, Institute for Basic Science, Pohang 37673, Korea;

Department of Mathematics, POSTECH, Pohang 37673, Korea; wlog@ postech.ac.kr

\section{Yuri Prokhorov}

Steklov Mathematical Institute of Russian Academy of Sciences, Moscow 119991, Russia;

National Research University Higher School of Economics, 6 Usacheva Street, Moscow 119048,

Russia; prokhoro@mi-ras.ru

\section{Mikhail Zaidenberg}

Institut Fourier, Université de Grenoble I, CS 40700, 38058 Grenoble cedex 09, France;

mikhail.zaidenberg@univ-grenoble-alpes.fr 